\title{
Asymmetric Claisen Rearrangements Enabled by Catalytic Asymmetric Di(allyl) Ether Synthesis
}

Supporting Information

\author{
Scott G. Nelson and Kan Wang
}

\section{TABLE OF CONTENTS}

$\begin{array}{ll}\text { General Procedure A: One-pot preparation of di(allyl) ethers } \mathbf{6} \text { and } \mathbf{8} & \text { page S1 } \\ \text { Compounds } \mathbf{6} \mathbf{a}-\mathbf{f} \text { and } \mathbf{8} & \text { page } \mathrm{S} 2-4 \\ \text { General Procedure B: Preparation of di(allyl) ethers } \mathbf{1 1} \text { from terminal alkynes } & \text { page S4 } \\ \text { Compounds 11a,b } & \text { page S5 } \\ \text { General Procedure C: ICR reactions of di(allyl)ethers } & \text { page S5 } \\ \text { Compounds 7a-f, 9, and 12-19 } & \text { page S6-14 } \\ { }^{1} \mathrm{H} \text { and }{ }^{13} \mathrm{C} \text { Spectra for all compounds } & \text { page S15-63 }\end{array}$

General Information: Unless otherwise stated, all reactions were performed in dry glassware under an atmosphere of oxygen-free nitrogen using standard inert atmosphere techniques for the manipulation of solvents and reagents. Anhydrous solvents were obtained by passage through successive alumina- and Q5-packed columns on a solvent purification system. Acetone was distilled from Drierite ${ }^{\circledR}$ and stored under dry nitrogen. $\quad\left[\left({ }^{C} \mathrm{C}_{8} \mathrm{H}_{14}\right)_{2} \mathrm{IrCl}\right]_{2}{ }^{1}$ and $\mathrm{PCy}_{3}$ were stored and weighed out in a nitrogen-filled glove box. Proton NMR chemical shifts are reported relative to residual $\mathrm{CHCl}_{3}(7.27 \mathrm{ppm})$ for ${ }^{1} \mathrm{H}$ and $\mathrm{CDCl}_{3}(77.00 \mathrm{ppm})$ for ${ }^{13} \mathrm{C} \mathrm{NMR}$ spectra. Flash chromatography was performed as previously described on Iatrobeads 6RS-8060 (Shell-USA) or EM silica gel 60 (230-240 mesh). ${ }^{2} \quad$ Analytical high performance liquid chromatography (HPLC) was performed using a variable wavelength UV detector (deuterium lamp, 190-600 nm) and Daicel Chiracel ${ }^{\mathrm{TM}}$ chiral stationary phase columns $(250 \times 4.6 \mathrm{~mm})$. HPLC grade isopropanol and hexanes were used as the eluting solvents.

General Procedure A: One-pot preparation of di(allyl) ethers 6: To a $0{ }^{\circ} \mathrm{C}$ solution of 1,7,7-trimethyl-3-morpholinobicyclo[2.2.1]heptan-2-ol [(-)-MIB] ${ }^{3}(2 \mathrm{~mol} \%, 0.02 \mathrm{mmol})$ in $2 \mathrm{ml}$ of toluene (pentane was used in certain trials) was added $\mathrm{Et}_{2} \mathrm{Zn}(1.0 \mathrm{M}$ in hexanes, 1.05 equiv, $1.05 \mathrm{ml})$

[1] A. L. Onderdelinden, A. van der Ent, Inorg. Chim. Acta 1972, 6, 420.

[2] W. C. Still, M. Kahn, A. Mitra, J. Org. Chem. 1978, 43, 2923-2925.

[3] W. A. Nugent, J. Chem. Soc., Chem. Commun. 1999, 1369-1370. 
via syringe. A solution of aldehyde $(1.0 \mathrm{mmol})$ in $1 \mathrm{ml}$ of toluene (or pentane) was added slowly ( $0.25 \mathrm{M}$ final concentration in aldehyde) and the reaction mixture was stirred at $0{ }^{\circ} \mathrm{C}$ until the reaction was complete as monitored by TLC ( $\sim 6 \mathrm{~h})$. A solution of acetic acid (1.05 equiv) in $2 \mathrm{ml}$ of THF was added dropwise and the resulting solution was stirred $10 \mathrm{~min}$. A solution of $\operatorname{Pd}(\mathrm{OAc})_{2}(5 \mathrm{~mol} \%)$, $\mathrm{PPh}_{3}(25 \mathrm{~mol} \%)$ and allyl acetate (1.5 equiv.) in $2 \mathrm{~mL}$ of THF ( $0.13 \mathrm{M}$ final concentration) was added and the reaction was heated at $60{ }^{\circ} \mathrm{C}$ for $12 \mathrm{~h}$. The reaction mixture was cooled to ambient temperature, the solvent was evaporated and the resulting crude product mixture was purified as indicated. Enantioselectivity for the $\mathrm{Et}_{2} \mathrm{Zn}$ addition was determined for the allylic alcohol obtained by quenching the $\mathrm{Et}_{2} \mathrm{Zn}$ addition reaction with saturated aqueous $\mathrm{NH}_{4} \mathrm{Cl}$ solution and extracting the resulting mixture with $\mathrm{Et}_{2} \mathrm{O}(3 \mathrm{x})$. The combined organic extracts were dried $\left(\mathrm{MgSO}_{4}\right)$, concentrated and the purified allylic alcohol was obtained using the indicated method.<smiles>C=CCOC(/C=C/c1ccccc1)CC</smiles>

$(\boldsymbol{S})$-(E)-1-Phenyl-3-allyloxypent-1-ene $(\mathbf{6 a}):^{4}$ General Procedure A was followed employing cinnamaldehyde $(1.0 \mathrm{mmol}, 132 \mathrm{mg})$. The crude product was purified by flash chromatograph on silical gel (2\% EtOAc in hexanes) to yield $151 \mathrm{mg}(75 \%)$ of the title compound as a light yellow oil. The allylic alcohol $(S, E)$-1-phenylpent-1-en-3-ol was purified by flash chromatograph on silica gel (2\% EtOAc in hexanes). Separating the allylic alcohol enantiomers by chiral HPLC [Daicel Chiracel ${ }^{\mathrm{TM}}$ OD-H column, flow rate $1.0 \mathrm{ml} / \mathrm{min}, 0.5 \% i$-PrOH, 99.5\% hexanes, $\mathrm{T}_{\mathrm{r}} 13.4(R)$ and $16.3(S)$ ] provided the enantiomer ratio: $3 R: 3 S=3.5: 96.5$ (93\% ee).

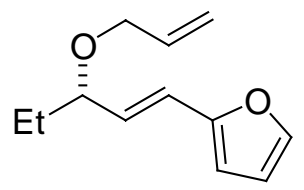

(3S)-(E)-2-(3-(Allyloxy)pent-1-enyl)furan (6b): General Procedure A was applied to $(E)$-3-(2-furyl)acrolein $(122 \mathrm{mg}, 1 \mathrm{mmol})$. The crude product was purified by column chromatography on silica gel (2\% EtOAc in hexanes) to yield $152 \mathrm{mg}$ $(79 \%)$ of the title compound as a light yellow oil. $[\alpha]_{\mathrm{D}}=-76.1$ (c 3.44, $\left.\mathrm{CHCl}_{3}\right)$; ${ }^{1} \mathrm{H}$ NMR $\left(300 \mathrm{MHz}, \mathrm{CDCl}_{3}\right): \delta 7.36(\mathrm{~d}, \mathrm{~J}=1.8 \mathrm{~Hz}, 1 \mathrm{H}), 6.38(\mathrm{dd}, \mathrm{J}=3.3,1.8 \mathrm{~Hz}, 1 \mathrm{H}), 6.37(\mathrm{~d}, \mathrm{~J}=$ $15.9 \mathrm{~Hz}, 1 \mathrm{H}), 6.25(\mathrm{~d}, \mathrm{~J}=3.2 \mathrm{~Hz}, 1 \mathrm{H}), 6.04(\mathrm{dd}, \mathrm{J}=15.9,7.7 \mathrm{~Hz}, 1 \mathrm{H}), 5.95$ (dddd, J = 17.2, 10.4, 6.0, $5.1 \mathrm{~Hz}, 1 \mathrm{H}), 5.30(\mathrm{dq}, \mathrm{J}=17.2,1.7 \mathrm{~Hz}, 1 \mathrm{H}), 5.18(\mathrm{dq}, \mathrm{J}=10.4,1.5 \mathrm{~Hz}, 1 \mathrm{H}), 4.10(\mathrm{ddt}, \mathrm{J}=12.8,5.1$, $1.5 \mathrm{~Hz}, 1 \mathrm{H}), 3.89$ (ddt, J = 12.8, 6.0, $1.4 \mathrm{~Hz}, 1 \mathrm{H}), 3.78(\mathrm{q}, \mathrm{J}=6.8 \mathrm{~Hz}, 1 \mathrm{H}), 1.55-1.78(\mathrm{~m}, 2 \mathrm{H}), 0.96(\mathrm{t}, \mathrm{J}$ $=7.4 \mathrm{~Hz}, 3 \mathrm{H}) \mathrm{ppm} ;{ }^{13} \mathrm{C} \mathrm{NMR}\left(75 \mathrm{MHz}, \mathrm{CDCl}_{3}\right): \delta .152 .31,144.81,135.14,129.14,120.20,116.46$, 111.17, 107.68, 81.01, 69.22, 28.58, 9.72 ppm; MS (EI, 70 eV): m/z $192\left(\mathrm{M}^{+}\right), 163,151,135,121,109$, 91, 81, 65, 57; HRMS $m / z$ calcd for $\mathrm{C}_{12} \mathrm{H}_{16} \mathrm{O}_{2}\left(\mathrm{M}^{+}\right):$192.1150. found 192.1153. The allylic alcohol $(S, E)$-1-(furan-2-yl)pent-1-en-3-ol was purified by column chromatography on silica gel (2\% EtOAc in hexanes); $[\alpha]_{\mathrm{D}}=-6.4\left(\mathrm{c} 1.74, \mathrm{CHCl}_{3}\right)$. Separating the enantiomers by chiral HPLC (Daicel Chiracel $^{\mathrm{TM}}$ OD-H column, flow rate $0.5 \mathrm{ml} / \mathrm{min}, 5 \% i$-PrOH, 95\% hexanes, $\mathrm{T}_{\mathrm{r}} 18.69(R)$ and $\left.20.52(S)\right)$ provided the enantiomer ratio: $3 R: 3 S=2.0: 98$ (96\% ee).

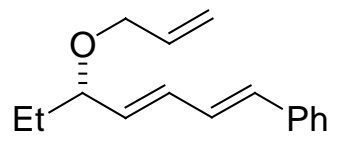

(5S)-(1E,3E)-5-Allyloxy-1-phenylhepta-1,3-diene (6c): General Procedure A was applied to $(2 E, 4 E)-5$-phenylpenta-2,4-dienal $(1.4 \mathrm{mmol}, 222 \mathrm{mg})$. The crude product was purified by column chromatography on silica gel (2\% EtOAc

[4] S. G. Nelson, C. J. Bungard, K. Wang, J. Am. Chem. Soc. 2003, 125, 13000-13001. 
in hexanes) to yield $278 \mathrm{mg}(87 \%)$ of the title compound as a light yellow oil. $[\alpha]_{\mathrm{D}}=-77.7$ (c 2.33, $\left.\mathrm{CHCl}_{3}\right) ;{ }^{1} \mathrm{H} \mathrm{NMR}\left(300 \mathrm{MHz}, \mathrm{CDCl}_{3}\right): \delta 7.22-7.45(\mathrm{~m}, 5 \mathrm{H}), 6.82(\mathrm{dd}, \mathrm{J}=15.7,10.5 \mathrm{~Hz}, 1 \mathrm{H}), 6.58(\mathrm{~d}, \mathrm{~J}$ $=15.7 \mathrm{~Hz}, 1 \mathrm{H}), 6.36(\mathrm{dd}, \mathrm{J}=15.3,10.4 \mathrm{~Hz}, 1 \mathrm{H}), 5.96(\mathrm{dddd}, \mathrm{J}=17.2,10.3,6.0,5.2 \mathrm{~Hz}, 1 \mathrm{H}), 5.70$ (dd, $\mathrm{J}=15.3,8.0 \mathrm{~Hz}, 1 \mathrm{H}), 5.31(\mathrm{dq}, \mathrm{J}=17.2,1.7 \mathrm{~Hz}, 1 \mathrm{H}), 5.19(\mathrm{dq}, \mathrm{J}=10.3,1.5 \mathrm{~Hz}, 1 \mathrm{H}), 4.10(\mathrm{ddt}, \mathrm{J}=$ 12.8, 5.2, $1.5 \mathrm{~Hz}, 1 \mathrm{H}), 3.89$ (ddt, J = 12.8, 6.0, $1.4 \mathrm{~Hz}, 1 \mathrm{H}), 3.75$ (q, J = 6.9 Hz, 1H), 1.55-1.78 (m, 2H), $0.96(\mathrm{t}, \mathrm{J}=7.4 \mathrm{~Hz}, 3 \mathrm{H}) \mathrm{ppm} ;{ }^{13} \mathrm{C} \mathrm{NMR}\left(75 \mathrm{MHz}, \mathrm{CDCl}_{3}\right): \delta$ 137.1, 135.2, 134.7, 132.6, 132.4, 128.6, 128.3, 127.5, 126.3, 116.5, 81.2, 69.2, 28.6, 9.8 ppm. MS (EI, $70 \mathrm{eV}): \mathrm{m} / z 228\left(\mathrm{M}^{+}\right), 199,187,171$, 157, 129, 115; HRMS $\mathrm{m} / z$ calcd for $\mathrm{C}_{16} \mathrm{H}_{20} \mathrm{O}\left(\mathrm{M}^{+}\right): 228.1514$; found 228.1532. The allylic alcohol (S,4E,6E)-7-phenylhepta-4,6-dien-3-ol was purified by flash chromatography on silica gel (2 \% EtOAc in hexanes); $[\alpha]_{\mathrm{D}}=+2.1$ (c 2.12, $\mathrm{CHCl}_{3}$ ). Separating the enantiomers by chiral HPLC (Daicel Chiracel $^{\mathrm{TM}}$ OD-H column, flow rate $1.0 \mathrm{ml} / \mathrm{min}, 10 \% i$-PrOH, $90 \%$ hexanes, $\mathrm{T}_{\mathrm{r}} 7.49(3 R)$ and $\left.9.57(3 S)\right)$ provided the enantiomer ratio: $3 R: 3 S=3.5: 96.5$ (93\% ee).

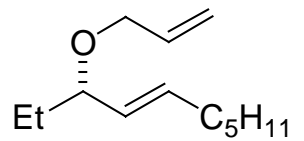

(3S)-(E)-3-(Allyloxy)dec-4-ene (6d): General Procedure A was applied to (E)-oct-2-enal $(1.0 \mathrm{mmol}, 126 \mathrm{mg})$ using pentane as the reaction solvent. The crude product was purified by column chromatography on silica gel (2\% EtOAc in hexanes) to yield $157 \mathrm{mg}(80 \%)$ of the title compound as a light yellow oil. $[\alpha]_{\mathrm{D}}=-13.6$ (c 3.77 , $\left.\mathrm{CHCl}_{3}\right) ;{ }^{1} \mathrm{H}$ NMR $\left(300 \mathrm{MHz}, \mathrm{CDCl}_{3}\right): \delta 5.85-5.98(\mathrm{~m}, 1 \mathrm{H}), 5.58(\mathrm{dt}, \mathrm{J}=15.4,6.7 \mathrm{~Hz}, 1 \mathrm{H}), 5.22-5.30$ $(\mathrm{m}, 2 \mathrm{H}), 5.15(\mathrm{~d}, \mathrm{~J}=10.3 \mathrm{~Hz}, 1 \mathrm{H}), 4.03(\mathrm{ddt}, \mathrm{J}=12.8,5.2,1.5 \mathrm{~Hz}, 1 \mathrm{H}), 3.81$ (ddt, J = 12.8, 6.0, $1.3 \mathrm{~Hz}$, $1 \mathrm{H}), 3.56(\mathrm{q}, \mathrm{J}=7.1 \mathrm{~Hz}, 1 \mathrm{H}), 2.05(\mathrm{q}, \mathrm{J}=7.0 \mathrm{~Hz}, 2 \mathrm{H}), 1.48-1.71(\mathrm{~m}, 2 \mathrm{H}), 1.26-1.50(\mathrm{~m}, 6 \mathrm{H}), 0.90(\mathrm{t}, \mathrm{J}$ $=7.0 \mathrm{~Hz}, 3 \mathrm{H}), 0.89(\mathrm{t}, \mathrm{J}=7.4 \mathrm{~Hz}, 3 \mathrm{H}) \mathrm{ppm} ;{ }^{13} \mathrm{C} \mathrm{NMR}\left(75 \mathrm{MHz}, \mathrm{CDCl}_{3}\right): \delta 135.4,134.3,130.5,116.3$, 81.8, 68.7, 32.7, 31.3, 28.9, 28.5, 22.5, 14.0, 9.9 ppm; MS (EI, $70 \mathrm{eV}): \mathrm{m} / z 167$ (M+Et), 125, 97, 67, 55; HRMS $\mathrm{m} / z$ calcd for $\mathrm{C}_{11} \mathrm{H}_{19} \mathrm{O}\left(\mathrm{M}^{+}-\mathrm{Et}\right)$ : 167.1436; found 167.1442. The allylic alcohol $(S, E)$-dec-4-en-3-ol $(15.6 \mathrm{mg}, 0.1 \mathrm{mmol})$ obtained by quenching the diethylzinc addition was reacted with $(R)$-2-methoxy-2-phenylacetic acid (18.3 mg, $0.11 \mathrm{mmol}), 1,3$-dicyclohexylcarbodimide $(22.7 \mathrm{mg}$, $0.11 \mathrm{mmol})$ and 4-(dimethylamino)pyridine $(1.3 \mathrm{mg}, 0.01 \mathrm{mmol})$ in $\mathrm{CH}_{2} \mathrm{Cl}_{2}$ (1 ml) for $12 \mathrm{~h}$ at ambient temperature. The solvent was evaportaed and the crude product was filtered through a pad of silical gel (4\% EtOAc in hexanes eluent) to yield $\left(2^{\prime} R\right)-(S, E)$-dec-4-en-3-yl 2'-methoxy-2'-phenylacetate. Separating the diastereomers by HPLC [Zorbax $(4.6 \mathrm{~mm} \mathrm{X} 25 \mathrm{~cm})$ column, flow rate $0.8 \mathrm{ml} / \mathrm{min}, 2 \%$ EtOAc, 98\% hexanes, $\mathrm{T}_{\mathrm{r}} 10.01(3 R)$ and $9.17(3 S)$ ] provided the diastereomer ratio: $3 R: 3 S=5.9: 94.1$ $(88 \%$ ee).

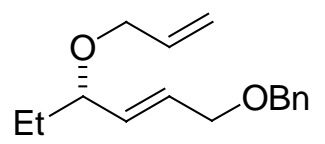

(4S)-(E)-4-Allyloxy-1-benzyloxyhex-2-ene (6e): General Procedure A was followed employing (E)-4-(benzyloxy)but-2-enal (176 mg, $1.0 \mathrm{mmol})$. The crude product was purified by flash chromatograph on silica gel (5\% EtOAc in hexanes) to yield $180 \mathrm{mg}(73 \%)$ of the title compound as a light yellow oil. $[\alpha]_{\mathrm{D}}=-26.9$ (c 2.87 , $\left.\mathrm{CHCl}_{3}\right) ;{ }^{1} \mathrm{H}$ NMR $\left(300 \mathrm{MHz}, \mathrm{CDCl}_{3}\right): \delta 7.20-7.38(\mathrm{~m}, 5 \mathrm{H}), 5.87-6.00(\mathrm{~m}, 1 \mathrm{H}), 5.78(\mathrm{dt}, \mathrm{J}=15.6,5.6$ $\mathrm{Hz}, 1 \mathrm{H}), 5.61(\mathrm{dd}, \mathrm{J}=15.6,7.6 \mathrm{~Hz}, 1 \mathrm{H}), 5.29(\mathrm{~d}, \mathrm{~J}=17.2 \mathrm{~Hz}, 1 \mathrm{H}), 5.18(\mathrm{~d}, \mathrm{~J}=10.4 \mathrm{~Hz}, 1 \mathrm{H}), 4.04-4.11$ $(\mathrm{m}, 3 \mathrm{H}), 3.87$ (ddt, J = 12.8, 5.9, $1.3 \mathrm{~Hz}, 1 \mathrm{H}), 3.69(\mathrm{q}, \mathrm{J}=6.8 \mathrm{~Hz}, 1 \mathrm{H}), 1.50-1.76(\mathrm{~m}, 2 \mathrm{H}), 0.94(\mathrm{t}, \mathrm{J}=$ $7.4 \mathrm{~Hz}, 3 \mathrm{H}) \mathrm{ppm} ;{ }^{13} \mathrm{C} \mathrm{NMR}\left(75 \mathrm{MHz}, \mathrm{CDCl}_{3}\right): \delta 138.16,135.11,133.72,129.24,128.32,127.66$, 127.55, 116.46, 80.86, 71.98, 70.05, 69.13, 28.32, 9.75; MS (EI, $70 \mathrm{eV}): \mathrm{m} / z 217\left(\mathrm{M}^{+}-\mathrm{Et}\right), 199,181$, 
$169,159,143,131,125,117,105$; HRMS $m / z$ calcd for $\mathrm{C}_{14} \mathrm{H}_{17} \mathrm{O}_{2}\left(\mathrm{M}^{+}-\mathrm{Et}\right): 217.1229$; found 217.1227. The allylic alcohol $(S, E)$-1-(benzyloxy)hex-2-en-4-ol was purified by flash chromatograph on silical gel $\left(5 \%\right.$ EtOAc in hexanes); $[\alpha]_{\mathrm{D}}=+5.8\left(\mathrm{c} 3.89, \mathrm{CHCl}_{3}\right)$. Separating the allylic alcohol enantiomers by chiral HPLC [Daicel Chiracel ${ }^{\mathrm{TM}}$ OD-H column, flow rate $0.5 \mathrm{ml} / \mathrm{min}, 5 \% i-\mathrm{PrOH}, 95 \%$ hexanes, $\mathrm{T}_{\mathrm{r}}$ $24.70(R)$ and $26.93(S)$ ] provided the enantiomer ratio: $4 R: 4 S=3.6: 96.4$ (93\% ee).

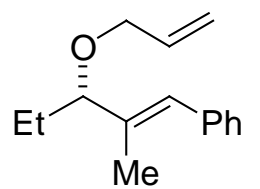

(3S)-(E)-3-Allyloxy-2-methyl-1-phenyl-1-pentene (6f): General Procedure A was applied to $(E)$-2-methyl-3-phenylacrylaldehyde $(1.0 \mathrm{mmol}, 146 \mathrm{mmol})$. The crude product was purified by column chromatography on silica gel (2\% EtOAc in hexanes) to yield $181 \mathrm{mg}(84 \%)$ of the title compound as a light yellow oil. $\quad[\alpha]_{\mathrm{D}}=+9.3(\mathrm{c}$ 5.48, $\left.\mathrm{CHCl}_{3}\right) ;{ }^{1} \mathrm{H}$ NMR $\left(300 \mathrm{MHz}, \mathrm{CDCl}_{3}\right): \delta$ 7.24-7.44 (m, 5H), $6.47(\mathrm{~s}, 1 \mathrm{H}), 5.93-6.05(\mathrm{~m}, 1 \mathrm{H}), 5.34$ $(\mathrm{d}, \mathrm{J}=17.2 \mathrm{~Hz}, 1 \mathrm{H}), 5.22(\mathrm{~d}, \mathrm{~J}=10.3 \mathrm{~Hz}, 1 \mathrm{H}), 4.06(\mathrm{ddt}, \mathrm{J}=12.8,5.1,1.4 \mathrm{~Hz}, 1 \mathrm{H}), 3.88$ (ddt, J = 12.8, $6.1,1.4 \mathrm{~Hz}, 1 \mathrm{H}), 3.75(\mathrm{t}, \mathrm{J}=6.9 \mathrm{~Hz}, 1 \mathrm{H}), 1.87(\mathrm{~d}, \mathrm{~J}=1.3 \mathrm{~Hz}, 3 \mathrm{H}), 1.55-1.84(\mathrm{~m}, 2 \mathrm{H}), 0.98(\mathrm{t}, \mathrm{J}=7.4$ $\mathrm{Hz}, 3 \mathrm{H}) \mathrm{ppm} ;{ }^{13} \mathrm{C} \mathrm{NMR}\left(75 \mathrm{MHz}, \mathrm{CDCl}_{3}\right): \delta 137.7,137.4,135.2,128.9,128.0,127.9,126.3,116.4$, 86.9, 68.9, 26.6, 12.3, 10.3 ppm; MS (EI, $70 \mathrm{eV}): \mathrm{m} / z 216\left(\mathrm{M}^{+}\right), 187,159,145,129,117,105,91,77$; HRMS $\mathrm{m} / \mathrm{z}$ calcd for $\mathrm{C}_{15} \mathrm{H}_{20} \mathrm{O} \quad\left(\mathrm{M}^{+}\right): 216.1514$; found 216.1507. The allylic alcohol ( $S, E)$-2-methyl-1-phenylpent-1-en-3-ol was purified by column chromatography on silica gel (2\% EtOAc in hexanes); $[\alpha]_{\mathrm{D}}=+37.6\left(\mathrm{c} 1.74, \mathrm{CHCl}_{3}\right)$. Separating the enantiomers by chiral HPLC (Daicel Chiracel $^{\mathrm{TM}}$ OD-H column, flow rate $0.6 \mathrm{ml} / \mathrm{min}, 3 \% i-\mathrm{PrOH}, 97 \%$ hexanes, $\mathrm{T}_{\mathrm{r}} 21.15(3 R)$ and $23.40(3 S)$ ) provided the enantiomer ratio: $3 R: 3 S=0.8: 99.2$ (98\% ee).

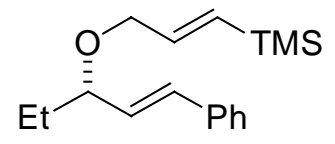

(1E)-\{3-[(3S)-(1E)-1-Phenylpent-1-en-3-yloxy]prop-1-enyl\}trimethylsilane (8): General Procedure A was applied to cinnamaldehyde $(1.0 \mathrm{mmol}, 132 \mathrm{mg})$ and using (E)-3-(trimethylsilyl)allyl acetate $(1.5 \mathrm{mmol}, 258 \mathrm{mg})$ in place of allyl acetate. The crude product was purified by column chromatography on silical gel (2\% EtOAc in hexanes) to yield $211 \mathrm{mg}(77 \%)$ of the title compound as a light yellow oil. $[\alpha]_{\mathrm{D}}=-71.3$ (c 3.57, $\left.\mathrm{CHCl}_{3}\right) ;{ }^{1} \mathrm{H} \mathrm{NMR}\left(300 \mathrm{MHz}, \mathrm{CDCl}_{3}\right): \delta 7.19-7.41(\mathrm{~m}, 5 \mathrm{H}), 6.51(\mathrm{~d}, \mathrm{~J}=15.9 \mathrm{~Hz}, 1 \mathrm{H}), 6.11(\mathrm{dt}, \mathrm{J}=18.7$, $4.8 \mathrm{~Hz}, 1 \mathrm{H}), 6.07(\mathrm{dd}, \mathrm{J}=15.9,8.0 \mathrm{~Hz}, 1 \mathrm{H}), 5.92(\mathrm{dt}, \mathrm{J}=18.7,1.4 \mathrm{~Hz}, 1 \mathrm{H}), 4.12(\mathrm{ddd}, \mathrm{J}=13.2,4.6$, $1.6 \mathrm{~Hz}, 1 \mathrm{H}), 3.92(\mathrm{ddd}, \mathrm{J}=13.2,5.1,1.4 \mathrm{~Hz}, 1 \mathrm{H}), 1.49-1.81(\mathrm{~m}, 2 \mathrm{H}), 0.96(\mathrm{t}, \mathrm{J}=7.4 \mathrm{~Hz}, 3 \mathrm{H}), 0.08$ (s, 9H) ppm; ${ }^{13} \mathrm{C}$ NMR $\left(75 \mathrm{MHz}, \mathrm{CDCl}_{3}\right): \delta 142.9,136.7,132.2,131.4,130.5,128.5,127.6,126.4,82.0$, 71.3, 28.6, 9.9, -1.4 ppm; MS (EI, $70 \mathrm{eV}): \mathrm{m} / \mathrm{z} 274\left(\mathrm{M}^{+}\right), 259,245,216,202,171,161,145,129,115$, $103,91,75$; HRMS $m / z$ calcd for $\mathrm{C}_{15} \mathrm{H}_{20} \mathrm{O}\left(\mathrm{M}^{+}\right)$: 274.1753; found 274.1766 .

General Procedure B: Preparation of di(allyl) ethers 11 from terminal alkynes: ${ }^{5}$ Cyclohexene $(2.0$ equiv.) was added to a solution of $\mathrm{BH}_{3} \cdot \mathrm{THF}\left(1.0 \mathrm{M}\right.$ in THF, 1.0 equiv.) at $0{ }^{\circ} \mathrm{C}$ and the resulting suspension was stirred $3 \mathrm{~h}$. The alkyne (1.0 equiv.) was added to the suspension of dicyclohexylborane and the reaction was stirred $15 \mathrm{~min}$ at $0{ }^{\circ} \mathrm{C}$ and $1 \mathrm{~h}$ at ambient temperature. The THF was evaporated in vacuo and the resulting slurry was dissolved in pentane (1.0 M solution) and

[5] For MIB-catalyzed additions of in situ generated vinyl zinc reagents to benzaldehyde, see: Y. K. Chen, A. E. Lurain, P. J. Walsh, J. Am. Chem. Soc. 2002, 124, 12225. 
the solution was cooled to $-78{ }^{\circ} \mathrm{C}$. Diethylzinc (1.0 M in hexanes, 1.05 equiv.) and (-)-MIB (2 mol $\%$ ) were added at $-78{ }^{\circ} \mathrm{C}$ and the reaction was stirred $30 \mathrm{~min}$ and then warmed to $0{ }^{\circ} \mathrm{C}$. A $1 \mathrm{M}$ pentane solution of benzaldehyde ( 0.9 equiv.) was added over $10 \mathrm{~min}$ and the reaction was stirred $3 \mathrm{~h}$ at $0{ }^{\circ} \mathrm{C}$. A $1 \mathrm{M}$ THF solution of acetic acid (1.05 equiv.) was added and the reaction stirred for 10 min. The resulting solution was added via syringe to a solution of palladium acetate $(5 \mathrm{~mol} \%), \mathrm{PPh}_{3}$ (25 mol \%) and allyl acetate (1.5 equiv.) in THF (0.1 M final concentration). The reaction was heated at $60{ }^{\circ} \mathrm{C}$ for $12 \mathrm{~h}$ then cooled to ambient temperature, the solvents were evaporated and the residue purified as indicated. Enantiomer ratios were determined for the allylic alcohol obtained by quenching the vinylzinc addition with saturatedaqueous $\mathrm{NH}_{4} \mathrm{Cl}$ solution was added to quench the reaction after alkenylzinc addition and extracting the resulting mixture with $\mathrm{Et}_{2} \mathrm{O}(3 \mathrm{x})$. The combined organic extracts were dried $\left(\mathrm{MgSO}_{4}\right)$, concentrated and the purified allylic alcohol was obtained using the indicated method.

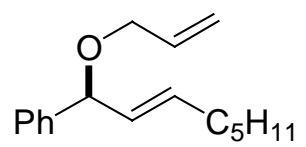

(1S)-(E)-1-Allyloxy-1-phenyl-2-octene (11a): General Procedure B was applied to 1-heptyne (2.0 mmol, $192 \mathrm{mg})$ and benzaldehyde $(1.8 \mathrm{mmol}, 191 \mathrm{mg})$. The crude product was purified by column chromatography on silical gel $(2 \%$ EtOAc in hexanes) to yield $302 \mathrm{mg}(62 \%)$ of the title compound as a light yellow oil. $[\alpha]_{\mathrm{D}}=25.3$ (c 2.74, $\left.\mathrm{CHCl}_{3}\right) ;{ }^{1} \mathrm{H}$ NMR $\left(300 \mathrm{MHz}, \mathrm{CDCl}_{3}\right): \delta$ 7.24-7.44 (m, 5H), 5.87-6.01 (m, 1H), $5.69(\mathrm{dt}, \mathrm{J}=15.3,6.4$ $\mathrm{Hz}, 1 \mathrm{H}), 5.56(\mathrm{dd}, \mathrm{J}=15.3,7.1 \mathrm{~Hz}, 1 \mathrm{H}), 5.27(\mathrm{~d}, \mathrm{~J}=17.2 \mathrm{~Hz}, 1 \mathrm{H}), 5.16(\mathrm{~d}, \mathrm{~J}=10.4 \mathrm{~Hz}, 1 \mathrm{H}), 4.76(\mathrm{~d}, \mathrm{~J}$ $=7.0 \mathrm{~Hz}, 1 \mathrm{H}), 4.00(\mathrm{dd}, \mathrm{J}=12.8,5.5 \mathrm{~Hz}, 1 \mathrm{H}), 3.93(\mathrm{dd}, \mathrm{J}=12.8,5.5 \mathrm{~Hz}, 1 \mathrm{H}), 2.04(\mathrm{q}, \mathrm{J}=7.0 \mathrm{~Hz}, 2 \mathrm{H})$, 1.27-1.43 (m, 6H), $0.87(\mathrm{t}, \mathrm{J}=7.2 \mathrm{~Hz}, 3 \mathrm{H}) ;{ }^{13} \mathrm{C} \mathrm{NMR}\left(75 \mathrm{MHz}, \mathrm{CDCl}_{3}\right): \delta 141.8,134.9,133.7,130.6$, 128.3, 127.3, 126.7, 116.6, 81.8, 68.9, 32.2, 31.3, 28.7, 22.4, 14.0 ppm; MS (EI, 70 eV): $m / z 244\left(\mathrm{M}^{+}\right)$, 203, 186, 173, 161, 143, 129, 117, 105, 99, 91, 84; HRMS $m / z$ calcd for $\mathrm{C}_{17} \mathrm{H}_{24} \mathrm{O}\left(\mathrm{M}^{+}\right): 244.1827$; found 244.1816. The allylic alcohol (S,E)-1-phenyloct-2-en-1-ol was purified by column chromatography on silical gel (2\% EtOAc in hexanes); $[\alpha]_{\mathrm{D}}=26.4$ (c 2.26, $\left.\mathrm{CHCl}_{3}\right)$. Separating the enantiomers by chiral HPLC (Daicel Chiracel ${ }^{\mathrm{TM}} \mathrm{OD}-\mathrm{H}$ column, flow rate $0.5 \mathrm{ml} / \mathrm{min}, 5.0 \% i$-PrOH, $95.0 \%$ hexanes, $\mathrm{T}_{\mathrm{r}} 14.53(1 R)$ and $18.27(1 S)$ provided the enantiomer ratio: $1 R: 1 S=6.7: 93.3(87 \%$ ee).

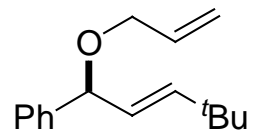

(1S)-(E)-1-Allyloxy-4,4-dimethyl-1-phenyl-2-pentene (11b): General Procedure B was applied to 3,3-dimethylbut-1-yne $(2.0 \mathrm{mmol}, 164 \mathrm{mg})$ and benzaldehyde (1.8 mmol, $191 \mathrm{mg})$. The crude product was purified by column chromatography on silical gel (2\% EtOAc in hexanes) to yield $290 \mathrm{mg}(63 \%)$ of the title compound as a light yellow oil. $\left[\alpha_{\mathrm{D}}=38.6\right.$ (c 2.07, $\left.\mathrm{CHCl}_{3}\right) ;{ }^{1} \mathrm{H}$ NMR $\left(300 \mathrm{MHz}, \mathrm{CDCl}_{3}\right): \delta 7.40-7.62(\mathrm{~m}, 5 \mathrm{H}), 6.10(\mathrm{ddt}, \mathrm{J}=17.2$, 10.4, $5.6 \mathrm{~Hz}, 1 \mathrm{H}), 5.87$ (dd, J = 15.6, $0.9 \mathrm{~Hz}, 1 \mathrm{H}), 5.62(\mathrm{dd}, \mathrm{J}=15.6,7.4 \mathrm{~Hz}, 1 \mathrm{H}), 5.43$ (dq, J = 17.2, $1.7 \mathrm{~Hz}, 1 \mathrm{H}), 5.33$ (dq, J = 10.4, $1.4 \mathrm{~Hz}, 1 \mathrm{H}), 4.91$ (d, J = 7.4 Hz, 1H), 4.15 (ddt, J = 12.9, 5.5, $1.3 \mathrm{~Hz}$, $1 \mathrm{H}), 4.08$ (ddt, $\mathrm{J}=12.9,5.6,1.3 \mathrm{~Hz}, 1 \mathrm{H}), 1.17$ (s, 9H) ppm; ${ }^{13} \mathrm{C}$ NMR $\left(75 \mathrm{MHz}, \mathrm{CDCl}_{3}\right): \delta 144.62$, 141.94, 135.03, 128.30, 127.30, 126.76, 125.40, 116.76, 82.01, 68.90, 33.00, 29.44 ppm; MS (EI, 70 $\mathrm{eV}): \mathrm{m} / z 230\left(\mathrm{M}^{+}\right), 188,173,157,147,131,115,105 ; \mathrm{HRMS} m / z$ calcd for $\mathrm{C}_{16} \mathrm{H}_{22} \mathrm{O}$ : 230.1671; found 230.1671. The allylic alcohol (S,E)-4,4-dimethyl-1-phenylpent-2-en-1-ol was purified by column chromatography on silical gel (2\% EtOAc in hexanes). Separating enantiomers by chiral HPLC 
(Daicel Chiracel ${ }^{\mathrm{TM}}$ OD-H column, flow rate $0.8 \mathrm{ml} / \mathrm{min}, 5.0 \% i$-PrOH, $95.0 \%$ hexanes, $\mathrm{T}_{\mathrm{r}} 9.3(1 R)$ and $11.8(1 S))$ provided the enantiomer ratio: $1 R: 1 S=4.1: 95.9$ (92\% ee).

General Procedure C: ICR reactions of di(allyl)ethers: ${ }^{4}$ A solution of $\left[\operatorname{IrCl}\left(\mathrm{C}_{8} \mathrm{H}_{14}\right)_{2}\right](0.005$ equiv) and $\mathrm{PCy}_{3}$ (0.03 equiv, 6:1 phosphine:Ir dimer) in anhydrous $\mathrm{CH}_{2} \mathrm{Cl}_{2}$ or 1,2-DCE (1.5 ml) was added to a solution of $\mathrm{NaBPh}_{4}\left(0.01\right.$ equiv, 2:1 NaBPh 4 :Ir dimer) in 25:1 $\mathrm{CH}_{2} \mathrm{Cl}_{2}$ /acetone or 1,2-DCE/acetone (1.5 ml, $0.67 \mathrm{M}$ final concentration in substrate) and the resulting yellow solution stirred for $5 \mathrm{~min}$ at ambient temperature. The diallyl ether (1.0 equiv) was added and the reaction stirred for 30 min (or indicated initial time) at ambient temperature whereupon $\mathrm{PPh}_{3}(0.03$ equiv, 6:1 phosphine:Ir dimer) was added and the resulting solution heated at reflux for the indicated time. The solvent was evaporated diastereomer ratio was determined for the crude aldehyde product by integrating the aldehyde resonances $(-\mathrm{C} \underline{\mathbf{H}} \mathrm{O})$ in the ${ }^{1} \mathrm{H}$ NMR spectrum. The crude product mixture was purified by column chromatography as indicated.

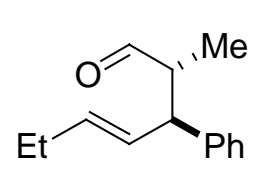

(2R,3S)-(E)-2-Methyl-3-phenylhept-4-enal (7a): General Procedure $\mathbf{C}$ was followed employing di(allyl) ether 6a $(202 \mathrm{mg}, 1.0 \mathrm{mmol})$ in 1,2-DCE solvent and an initial reaction time of $10 \mathrm{~min}$. The reaction was heated at reflux for $9 \mathrm{~h} ;(2 R, 3 S):(2 S: 3 R)=$ 95:5. Purification by flash chromatograph on Iatrobeads neutral $(\mathrm{pH} 7)$ silica gel (2.5\% $\mathrm{Et}_{2} \mathrm{O}$ in hexanes) to yielded $156 \mathrm{mg}(77 \%)$ of the title compound as a colorless oil. Separating the enantiomers by chiral HPLC (Daicel Chiracel ${ }^{\mathrm{TM}}$ OD-H column, flow rate $1.0 \mathrm{ml} / \mathrm{min}$, $1.0 \% i-\mathrm{PrOH}, 99.0 \%$ hexanes, $\mathrm{T}_{\mathrm{r}} 9.12(2 S, 3 R)$ and $10.33(2 R, 3 S)$ provided the enantiomer ratio: $(2 R$, $3 S):(2 S: 3 R)=95.5: 3.5(92 \%$ ee $) . \quad[\alpha]_{\mathrm{D}}=42.7\left(\mathrm{c} 2.35, \mathrm{CHCl}_{3}\right) ;{ }^{1} \mathrm{H} \mathrm{NMR}\left(300 \mathrm{MHz}, \mathrm{CDCl}_{3}\right): \delta 9.77$ $(\mathrm{d}, \mathrm{J}=3.2 \mathrm{~Hz}, 1 \mathrm{H}), 7.27-7.46(\mathrm{~m}, 5 \mathrm{H}), 5.72(\mathrm{dd}, \mathrm{J}=15.8,7.4 \mathrm{~Hz}, 1 \mathrm{H}), 5.65$ (dt, J = 15.8, $5.5 \mathrm{~Hz}, 1 \mathrm{H})$, $3.55(\mathrm{dd}, \mathrm{J}=9.4,7.3 \mathrm{~Hz}, 1 \mathrm{H}), 2.83(\mathrm{dqd}, \mathrm{J}=9.4,6.9,3.2 \mathrm{~Hz}, 1 \mathrm{H}), 2.06-2.15(\mathrm{~m}, 2 \mathrm{H}), 1.04(\mathrm{t}, \mathrm{J}=7.5$ $\mathrm{Hz}, 3 \mathrm{H}), 0.99(\mathrm{~d}, \mathrm{~J}=6.9 \mathrm{~Hz}, 3 \mathrm{H}) \mathrm{ppm} ;{ }^{13} \mathrm{C} \mathrm{NMR}\left(75 \mathrm{MHz}, \mathrm{CDCl}_{3}\right): \delta 204.9,141.8,134.2,129.6$, 128.6, 127.9, 126.6, 50.9, 50.7, 25.5, 13.5, 12.6 ppm; MS (EI, $70 \mathrm{eV}): \mathrm{m} / z 202\left(\mathrm{M}^{+}\right), 187,173,159$, $146,128,118,105,92,77$; HRMS $m / z$ calcd for $\mathrm{C}_{14} \mathrm{H}_{18} \mathrm{O}\left(\mathrm{M}^{+}\right): 202.1358$. found 202.1361 .

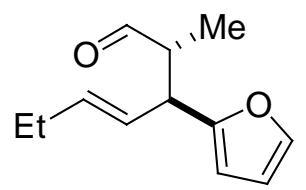

(2R,3S)-(E)-3-(Furan-2-yl)-2-methylhept-4-enal (7b): General Procedure C was followed employing di(allyl) ether $\mathbf{6 b}(130 \mathrm{mg}, 0.68 \mathrm{mmol})$ in 1,2-DCE solvent and an initial reaction time of $30 \mathrm{~min}$. The reaction was heated at reflux for $16 \mathrm{~h}$; $(2 R, 3 S):(2 S, 3 R)=95: 5$. Purification by flash chromatograph on Iatrobeads neutral (pH 7) silica gel $\left(2.5 \% \mathrm{Et}_{2} \mathrm{O}\right.$ in hexanes) yielded $97 \mathrm{mg}(75 \%)$ of the title compound as a colorless oil. Separating the enantiomers by chiral HPLC (Daicel Chiracel ${ }^{\mathrm{TM}}$ OD-H column, flow rate $0.8 \mathrm{ml} / \mathrm{min}$, $0.8 \% \mathrm{i}$-PrOH, 99.2\% hexanes, $\mathrm{T}_{\mathrm{r}} 8.45(2 S, 3 R)$ and $10.12(2 R, 3 S)$ provided the enantiomer ratio: $(2 R, 3 S):(2 S, 3 R)=98: 2(96 \%$ ee $) . \quad[\alpha]_{\mathrm{D}}=75.6\left(\mathrm{c} 0.66, \mathrm{CHCl}_{3}\right) ;{ }^{1} \mathrm{H} \mathrm{NMR}\left(300 \mathrm{MHz}, \mathrm{CDCl}_{3}\right): \delta 9.69$ $(\mathrm{d}, \mathrm{J}=1.9 \mathrm{~Hz}, 1 \mathrm{H}), 7.33(\mathrm{dd}, \mathrm{J}=1.9,0.9 \mathrm{~Hz}, 1 \mathrm{H}), 6.29(\mathrm{dd}, \mathrm{J}=3.2,1.9 \mathrm{~Hz}, 1 \mathrm{H}), 6.06(\mathrm{dt}, \mathrm{J}=3.2,0.8$ $\mathrm{Hz}, 1 \mathrm{H}), 5.65(\mathrm{dt}, \mathrm{J}=15.3,5.9 \mathrm{~Hz}, 1 \mathrm{H}), 5.54(\mathrm{ddt}, \mathrm{J}=15.3,8.2,1.2 \mathrm{~Hz}, 1 \mathrm{H}), 3.71(\mathrm{dd}, \mathrm{J}=8.2,6.6 \mathrm{~Hz}$, $1 \mathrm{H}), 2.78$ (qdd, J = 7.0, 6.6, $1.9 \mathrm{~Hz}, 1 \mathrm{H}), 1.00(\mathrm{~d}, \mathrm{~J}=7.0 \mathrm{~Hz}, 3 \mathrm{H}), 0.98(\mathrm{t}, \mathrm{J}=7.5 \mathrm{~Hz}, 3 \mathrm{H}) \mathrm{ppm} ;{ }^{13} \mathrm{C}$ NMR (75 MHz, $\left.\mathrm{CDCl}_{3}\right): \delta 204.0,155.0,141.5,136.8,125.9,110.1,106.3,49.7,43.8,25.4,13.5,11.4$ ppm; MS (EI, $70 \mathrm{eV}): \mathrm{m} / z, 192\left(\mathrm{M}^{+}\right), 163,135,117$; HRMS $m / z$ calcd for $\mathrm{C}_{12} \mathrm{H}_{16} \mathrm{O}_{2}\left(\mathrm{M}^{+}\right): 192.1150$; 
found 192.1146 .

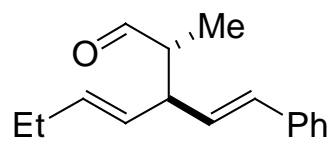

(2R,3R)-(4E)-2-Methyl-3-[(E)-styryl]hept-4-enal (7c): General Procedure C was followed employing di(allyl) ether $6 \mathbf{c}(228 \mathrm{mg}, 1.0 \mathrm{mmol})$ in $\mathrm{CH}_{2} \mathrm{Cl}_{2}$ solvent and an initial reaction time of $10 \mathrm{~min}$. The reaction was heated at reflux for 18 $\mathrm{h} ;(2 R, 3 R):(2 S, 3 S)=95: 5$. Purification by flash chromatograph on Iatrobeads neutral ( $\mathrm{pH} 7)$ silica gel ( $2 \%$ EtOAc in hexanes) yielded $176 \mathrm{mg}(77 \%)$ of the title compound as a colorless oil. Separating the enantiomers by chiral HPLC (Daicel Chiracel ${ }^{\mathrm{TM}} \mathrm{OD}-\mathrm{H}$ column, flow rate $0.8 \mathrm{ml} / \mathrm{min}, 1.0 \% i$-PrOH, 99.0\% hexanes, $\mathrm{T}_{\mathrm{r}} 14.03(2 S, 3 S)$ and $20.67(2 R, 3 R)$ provided the enantiomer ratio: $(2 S, 3 S):(2 R, 3 R)=$ 3.7:96.3 (93\% ee). $\quad[\alpha]_{\mathrm{D}}=54.8\left(\mathrm{c} 3.48, \mathrm{CHCl}_{3}\right) ;{ }^{1} \mathrm{H} \mathrm{NMR}\left(300 \mathrm{MHz}, \mathrm{CDCl}_{3}\right): \delta 9.69(\mathrm{~d}, \mathrm{~J}=2.3 \mathrm{~Hz}$, $1 \mathrm{H}), 6.44(\mathrm{~d}, \mathrm{~J}=15.9 \mathrm{~Hz}, 1 \mathrm{H}), 6.12(\mathrm{dd}, \mathrm{J}=15.8,8.1 \mathrm{~Hz}, 1 \mathrm{H}), 5.60$ (dt, J = 15.4, 6.1 Hz, 1H), 5.45 (dd, $\mathrm{J}=15.4,7.5 \mathrm{~Hz}, 1 \mathrm{H}), 3.22(\mathrm{q}, \mathrm{J}=7.5 \mathrm{~Hz}, 1 \mathrm{H}), 2.50(\mathrm{ddqd}, \mathrm{J}=8.1,7.5,7.0,2.3 \mathrm{~Hz}, 1 \mathrm{H}), 1.11(\mathrm{~d}, \mathrm{~J}=$ $7.0 \mathrm{~Hz}, 3 \mathrm{H}), 0.99$ (t, J = 7.4 Hz, 3H) ppm; ${ }^{13} \mathrm{C}$ NMR (75 MHz, $\left.\mathrm{CDCl}_{3}\right): \delta 204.7,137.1,134.7,131.4$, 129.5, 128.5, 128.5, 128.2, 127.3, 126.2, 50.4, 47.4, 25.6, 13.7, 11.4 ppm; MS (EI, $70 \mathrm{eV}): \mathrm{m} / \mathrm{z} 228$, 213, 199, 171, 143, 129, 115; HRMS m/z calcd for $\mathrm{C}_{16} \mathrm{H}_{20} \mathrm{O}\left(\mathrm{M}^{+}\right)$: 228.1514; found 228.1509.

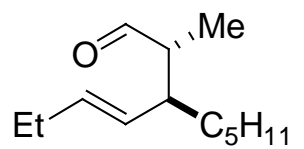

(2R,3S)-3-[(E)-But-1-enyl]-2-methyloctanal (7d): General Procedure C was followed employing di(allyl) ether $\mathbf{6 d}$ (392 $\mathrm{mg}, 2 \mathrm{mmol}$ ) in 1,2-DCE solvent and an initial reaction time of $10 \mathrm{~min}$. The reaction was heated at reflux for $18 \mathrm{~h}$; $(2 R, 3 S):(2 S, 3 R)=97: 3$. Purification by flash chromatograph on Iatrobeads neutral (pH 7) silica gel ( $2 \%$ EtOAc in hexanes) to yield $274 \mathrm{mg}(70 \%)$ of the title compound as a colorless oil. Separating the enantiomers by chiral GC (Chiracel G-TA column $(30 \mathrm{~m} \times 0.25 \mathrm{~mm}$ ), flow rate $0.6 \mathrm{~mL} / \mathrm{min}$, method: $50{ }^{\circ} \mathrm{C}$ for $2.00 \mathrm{~min}$, ramp @ $2.00{ }^{\circ} \mathrm{C} / \mathrm{min}$ to $160{ }^{\circ} \mathrm{C}$, hold for $20.00 \mathrm{~min}, \mathrm{~T}_{\mathrm{r}} 7.17(2 S, 3 R)$ and $9.22(2 R, 3 S)$ provided the enantiomer ratio: $(2 R, 3 S):(2 S, 3 R)=93.5: 6.5(87 \%$ ee $) . \quad[\alpha]_{\mathrm{D}}=10.6(\mathrm{c}$ 0.71, $\left.\mathrm{CHCl}_{3}\right) ;{ }^{1} \mathrm{H}$ NMR $\left(300 \mathrm{MHz}, \mathrm{CDCl}_{3}\right): \delta 9.64(\mathrm{~d}, \mathrm{~J}=2.2 \mathrm{~Hz}, 1 \mathrm{H}), 5.50(\mathrm{dt}, \mathrm{J}=15.3,6.4 \mathrm{~Hz}, 1 \mathrm{H})$, 5.21 (ddt, $\mathrm{J}=15.3,8.9,1.4 \mathrm{~Hz}, 1 \mathrm{H}), 2.21-2.38(\mathrm{~m}, 2 \mathrm{H}), 1.97-2.07(\mathrm{~m}, 2 \mathrm{H}), 1.11-1.40(\mathrm{~m}, 9 \mathrm{H}), 1.04(\mathrm{~d}$, $\mathrm{J}=5.9 \mathrm{~Hz}, 3 \mathrm{H}), 0.97(\mathrm{t}, \mathrm{J}=7.4 \mathrm{~Hz}, 3 \mathrm{H}), 0.88(\mathrm{t}, \mathrm{J}=7.4 \mathrm{~Hz}, 3 \mathrm{H}) \mathrm{ppm} ; ;{ }^{13} \mathrm{C} \mathrm{NMR}\left(75 \mathrm{MHz}, \mathrm{CDCl}_{3}\right): \delta$ 205.7, 134.4, 129.8, 50.7, 44.3, 31.7, 31.6, 26.8, 25.9, 22.5, 14.0, 13.9, 11.0 ppm; MS (EI, $70 \mathrm{eV}): \mathrm{m} / \mathrm{z}$ $196\left(\mathrm{M}^{+}\right), 167,153,139,125,97,83,73$; HRMS $\mathrm{m} / z$ calcd for $\mathrm{C}_{13} \mathrm{H}_{24} \mathrm{O}\left(\mathrm{M}^{+}\right)$: 196.1827; found 196.1825 .

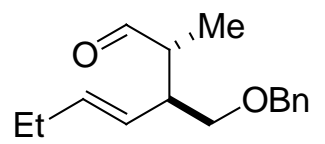

(2R,3S)-(E)-3-Benzyloxymethyl-2-methylhept-4-enal (7e): General Procedure C was followed employing di(allyl) ether $\mathbf{6 e}(123 \mathrm{mg}, 0.5 \mathrm{mmol})$ in 1,2-DCE solvent and an initial reaction time of $10 \mathrm{~min}$. The reaction was heated at reflux for $16 \mathrm{~h}$; $(2 R, 3 S):(2 S, 3 R)=96: 4$. Purification by flash chromatograph on Iatrobeads neutral (pH 7) silica gel $\left(2.5 \% \mathrm{Et}_{2} \mathrm{O}\right.$ in hexanes) yielded $74 \mathrm{mg}(60 \%)$ of the title compound as a colorless oil. Separating the enantiomers by chiral HPLC (Daicel Chiracel ${ }^{\mathrm{TM}} \mathrm{OD}-\mathrm{H}$ column, flow rate $0.8 \mathrm{ml} / \mathrm{min}, 0.8 \% i$-PrOH, 99.2\% hexanes, $\mathrm{T}_{\mathrm{r}} 20.27(2 S, 3 R)$ and $24.71(2 R, 3 S)$ provided the enantiomer ratio: $(2 S: 3 R):(2 R, 3 S)=$ 4.1:95.9 (92\% ee). $[\alpha]_{\mathrm{D}}=53.6\left(\mathrm{c} 1.51, \mathrm{CHCl}_{3}\right) ;{ }^{1} \mathrm{H}$ NMR $\left(300 \mathrm{MHz}, \mathrm{CDCl}_{3}\right): \delta 9.70(\mathrm{~d}, \mathrm{~J}=1.2 \mathrm{~Hz}$, $1 \mathrm{H}), 7.27-7.61(\mathrm{~m}, 5 \mathrm{H}), 5.60(\mathrm{dt}, \mathrm{J}=15.4,6.3 \mathrm{~Hz}, 1 \mathrm{H}), 5.35(\mathrm{dd}, \mathrm{J}=15.4,8.3 \mathrm{~Hz}, 1 \mathrm{H}), 4.48(\mathrm{~s}, 2 \mathrm{H})$, $3.50(\mathrm{dd}, \mathrm{J}=9.3,5.8 \mathrm{~Hz}, 1 \mathrm{H}), 3.45(\mathrm{dd}, \mathrm{J}=9.3,7.9 \mathrm{~Hz}, 1 \mathrm{H}), 2.79-2.87(\mathrm{~m}, 1 \mathrm{H}), 2.48-2.56(\mathrm{~m}, 1 \mathrm{H})$, 
$2.03(\mathrm{qd}, \mathrm{J}=7.4,6.3 \mathrm{~Hz}, 2 \mathrm{H}), 1.06(\mathrm{~d}, \mathrm{~J}=6.9 \mathrm{~Hz}, 3 \mathrm{H}), 0.98(\mathrm{t}, \mathrm{J}=7.4 \mathrm{~Hz}, 3 \mathrm{H}) \mathrm{ppm} ;{ }^{13} \mathrm{C}$ NMR $(75$ $\mathrm{MHz}, \mathrm{CDCl}_{3}$ ): $\delta$ 204.7, 138.2, 135.3, 128.4, 127.6 (3C, overlap), 126.6, 73.2, 70.7, 47.9, 44.2, 25.7, 13.7, $9.9 \mathrm{ppm}$; MS (EI, $70 \mathrm{eV}): \mathrm{m} / z 246\left(\mathrm{M}^{+}\right), 188,158,125,105,91,81,65$; HRMS $\mathrm{m} / \mathrm{z}$ calcd for $\mathrm{C}_{16} \mathrm{H}_{22} \mathrm{O}_{2}\left(\mathrm{M}^{+}\right)$: 246.1620; found 246.1622 .

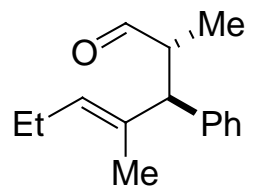

(2R,3R)-(E)-2,4-Dimethyl-3-phenylhept-4-enal (7f): General Procedure $\mathrm{C}$ was followed employing di(allyl) ether $\mathbf{6 f}(432 \mathrm{mg}, 2.0 \mathrm{mmol})$ in $\mathrm{CH}_{2} \mathrm{Cl}_{2}$ solvent and an initial reaction time of $10 \mathrm{~min}$. After the initial reaction time, triphenylphosphine (3 mol\%) was added, the $\mathrm{CH}_{2} \mathrm{Cl}_{2}$ solvent was evaporated, and the residue was dissolved in toluene $(5 \mathrm{~mL})$. The resulting solution was heated at reflux for $18 \mathrm{~h} ;\left(2 R_{E}, 3 R_{E}\right):\left(2 S_{E}, 3 S_{E}\right): \mathrm{Z}_{\mathrm{C} 4-5}=\mathrm{dr}$ $=76.4: 6.6: 17.0$. Purification by flash chromatograph on Iatrobeads neutral $(\mathrm{pH} 7)$ silica gel $\left(2 \% \mathrm{Et}_{2} \mathrm{O}\right.$ in hexanes) yielded $350 \mathrm{mg}(81 \%)$ of the title compound as a colorless oil. Separating the enantiomers by chiral HPLC (Daicel Chiracel ${ }^{\mathrm{TM}}$ OD-H column, flow rate $0.8 \mathrm{ml} / \mathrm{min}, 0.8 \% i$-PrOH, 99.2\% hexanes, $\mathrm{T}_{\mathrm{r}} 7.90(2 S, 3 S)$ and $11.4(2 R, 3 R)$ provided the enantiomer ratio: $(2 R, 3 R):(2 S, 3 S)=$ 98.5:1.5 (97\% ee). $\quad[\alpha]_{\mathrm{D}}=-36.5\left(\mathrm{c} 6.23, \mathrm{CHCl}_{3}\right) .{ }^{1} \mathrm{H}$ NMR $\left(300 \mathrm{MHz}, \mathrm{CDCl}_{3}\right): \delta 9.58(\mathrm{~d}, \mathrm{~J}=3.9 \mathrm{~Hz}$, $1 \mathrm{H}), 7.18-7.34(\mathrm{~m}, 5 \mathrm{H}), 5.43(\mathrm{t}, \mathrm{J}=7.0 \mathrm{~Hz}, 1 \mathrm{H}), 3.34(\mathrm{~d}, \mathrm{~J}=11.4 \mathrm{~Hz}, 1 \mathrm{H}), 2.90-3.01(\mathrm{~m}, 1 \mathrm{H})$, 2.05-2.18 (m, 2H), 1.59 (s, 3H), $1.04(\mathrm{t}, \mathrm{J}=7.4 \mathrm{~Hz}, 2 \mathrm{H}), 0.98(\mathrm{~d}, \mathrm{~J}=6.8 \mathrm{~Hz}, 3 \mathrm{H}) \mathrm{ppm} ;{ }^{13} \mathrm{C}$ NMR $(75$ $\left.\mathrm{MHz}, \mathrm{CDCl}_{3}\right): \delta 204.3,140.6,135.1,129.5,128.6,128.4,128.3,128.1,126.6,56.1,47.7,21.1,15.0$, 14.0, 13.4 ppm; MS (EI, $70 \mathrm{eV}): \mathrm{m} / z 216\left(\mathrm{M}^{+}\right), 201,187,173,159,143,131,117,105,91$; HRMS $\mathrm{m} / z$ calcd for $\mathrm{C}_{15} \mathrm{H}_{20} \mathrm{O}\left(\mathrm{M}^{+}\right)$: 216.1514; found 216.1502.

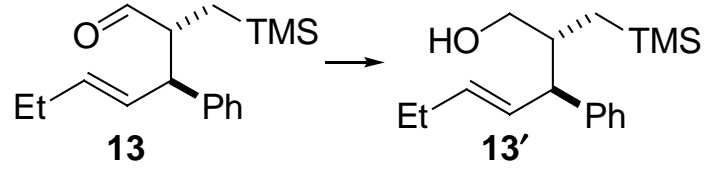

$(2 R, 3 S)-(E)$-2-(Trimethylsilylmethyl)-3-phenylhept-4-en al (9): General Procedure $\mathbf{C}$ was followed employing di(allyl) ether 8 (137 mg, $0.5 \mathrm{mmol})$ in 1,2-DCE solvent and an initial reaction time of $20 \mathrm{~h}$. The reaction was heated at reflux for $12 \mathrm{~h} ;(2 R, 3 S):(2 S, 3 R)=95: 5$. Due to the sensitivity of aldehyde 13 toward epimerization, the Claisen product was reduced to the corresponding primary alcohol that was characterized fully. Thus, the crude product mixture was dissolved in anhydrous $\mathrm{CH}_{2} \mathrm{Cl}_{2}$ and cooled to $-78{ }^{\circ} \mathrm{C}$ and a $1 \mathrm{M}$ hexanes solution of ${ }^{i} \mathrm{Bu}_{2} \mathrm{AlH}(0.6 \mathrm{ml}, 0.6 \mathrm{mmol})$ was added dropwise via syringe and the resulting solution was stirred for $5 \mathrm{~min}$ at $-78{ }^{\circ} \mathrm{C}$. The reaction mixture was warmed to ambient temperature and poured into $\mathrm{H}_{2} \mathrm{O}$. This mixture was diluted with EtOAc and $1 \mathrm{M} \mathrm{HCl}$, the layers were shaken and separated and the aqueous portion was extracted with EtOAc (3x). The combined organic extracts were dried $\left(\mathrm{MgSO}_{4}\right)$, filtered and the solvent was evaporated. The crude product was purified by flash chromatograph on silica gel (16\% EtOAc in hexanes) to yield $70 \mathrm{mg}$ $(50 \%)$ of $(2 R, 3 S)-(E)-2$-(trimethylsilylmethyl)-3-phenyl-4-hepten-1-ol $\left(\mathbf{9}^{\prime}\right)$ as a colorless oil. Separating the enantiomers by chiral HPLC (Daicel Chiracel ${ }^{\mathrm{TM}}$ OD-H column, flow rate $0.5 \mathrm{ml} / \mathrm{min}$, 5.0\% $i$ - $\mathrm{PrOH}, 95.0 \%$ hexanes, $\mathrm{T}_{\mathrm{r}} 7.08(2 S, 3 R)$ and $8.68(2 R, 3 S)$ provided the enantiomer ratio: $(2 R, 3 S):(2 S, 3 R)=95.5: 4.5(91 \%$ ee $) . \quad[\alpha]_{\mathrm{D}}=39.1\left(\mathrm{c} 2.20, \mathrm{CHCl}_{3}\right) ;{ }^{1} \mathrm{H}$ NMR $\left(300 \mathrm{MHz}, \mathrm{CDCl}_{3}\right): \delta$ 7.19-7.34 (m, 5H), $5.71(\mathrm{dd}, \mathrm{J}=15.3,8.6 \mathrm{~Hz}, 1 \mathrm{H}), 5.62(\mathrm{dt}, \mathrm{J}=15.3,5.7 \mathrm{~Hz}, 1 \mathrm{H}), 3.73(\mathrm{dd}, \mathrm{J}=11.1$, $3.7 \mathrm{~Hz}, 1 \mathrm{H}), 3.55(\mathrm{dd}, \mathrm{J}=11.1,5.6 \mathrm{~Hz}, 1 \mathrm{H}), 3.29(\mathrm{t}, \mathrm{J}=8.4 \mathrm{~Hz}, 1 \mathrm{H}), 2.03-2.08(\mathrm{~m}, 3 \mathrm{H}), 1.60$ (brs, $1 \mathrm{H})$, $0.43-0.52(\mathrm{~m}, 2 \mathrm{H}),-0.03$ (s, 9H) ppm; ${ }^{13} \mathrm{C} \mathrm{NMR}\left(75 \mathrm{MHz}, \mathrm{CDCl}_{3}\right): \delta 144.0,133.1,131.7,128.4,128.2$, 
$126.1,65.5,54.3,42.3,25.5,16.1,13.7,-0.8 \mathrm{ppm}$. Satisfactory mass spectral data was not obtained for the alcohol $\mathbf{1 3}^{\prime}$; copies of the ${ }^{1} \mathrm{H}$ and ${ }^{13} \mathrm{C}$ spectra are provided as evidence of compound composition and purity.

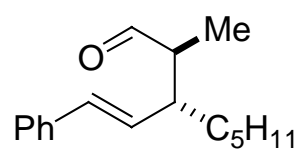

(2S,3R)-2-Methyl-3-(E)-styryloctanal (12a): General Procedure $\mathrm{C}$ was followed employing di(allyl) ether 11a $(244 \mathrm{mg}, 1.0 \mathrm{mmol})$ in dichloromethane solvent and an initial reaction time of $10 \mathrm{~min}$. The reaction was heated at reflux for $16 \mathrm{~h}$; $(2 S, 3 R):(2 R, 3 S)=95: 5$. Purification by flash chromatography on Iatrobeads neutral $(\mathrm{pH} 7)$ silica gel $\left(2.5 \% \mathrm{Et}_{2} \mathrm{O}\right.$ in hexanes) yielded $195 \mathrm{mg}(80 \%)$ of the title compound as a colorless oil. Separating the enantiomers by chiral HPLC (Daicel Chiracel ${ }^{\mathrm{TM}}$ OD-H column, flow rate $1.0 \mathrm{ml} / \mathrm{min}, 0.8 \% i$-PrOH, 99.2\% hexanes, $\mathrm{T}_{\mathrm{r}} 10.1(2 R, 3 S)$ and $15.1(2 S, 3 R)$ provided the enantiomer ratio: $(2 R, 3 S):(2 S, 3 R)=6.3$ : $92.7(86 \%$ ee $) . \quad[\alpha]_{\mathrm{D}}=-27.6\left(\mathrm{c} 2.54, \mathrm{CHCl}_{3}\right) ;{ }^{1} \mathrm{H} \mathrm{NMR}\left(300 \mathrm{MHz}, \mathrm{CDCl}_{3}\right): \delta 9.73(\mathrm{~d}, \mathrm{~J}=1.9 \mathrm{~Hz}, 1 \mathrm{H})$, 7.23-7.50 (m, 5H), $6.44(\mathrm{~d}, \mathrm{~J}=15.8,1 \mathrm{H}), 6.06(\mathrm{dd}, \mathrm{J}=15.8,9.0 \mathrm{~Hz}, 1 \mathrm{H}), 2.43-2.60(\mathrm{~m}, 2 \mathrm{H}), 1.25-1.49$ $(\mathrm{m}, 6 \mathrm{H}), 1.14(\mathrm{~d}, \mathrm{~J}=6.8 \mathrm{~Hz}, 3 \mathrm{H}), 0.90(\mathrm{t}, \mathrm{J}=7.4 \mathrm{~Hz}, 3 \mathrm{H}) ;{ }^{13} \mathrm{C} \mathrm{NMR}\left(75 \mathrm{MHz}, \mathrm{CDCl}_{3}\right): \delta 204.8,137.1$, 131.8, 131.2, 128.5, 127.2, 126.1, 50.7, 44.7, 31.7, 31.6, 27.0, 22.4, 14.0, 11.0 ppm; MS (EI, 70 eV): $\mathrm{m} / \mathrm{z} 244\left(\mathrm{M}^{+}\right), 187,173,145,129,115,104,91$; HRMS $\mathrm{m} / \mathrm{z}$ calcd for $\mathrm{C}_{17} \mathrm{H}_{24} \mathrm{O}: 244.1827$; found 244.1826 .

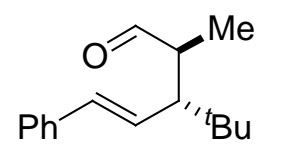

(2S,3R)-(E)-3-tert-Butyl-2-methyl-5-phenylpent-4-enal (12b): General Procedure C was followed employing di(allyl) ether $11 \mathbf{b}(125 \mathrm{mg}, 0.5 \mathrm{mmol})$ in 1,2-DCE solvent and an initial reaction time of $30 \mathrm{~min}$. The reaction was heated at reflux; $(2 \mathrm{~S}, 3 \mathrm{R}):(2 \mathrm{R}, 3 \mathrm{~S})=92: 8$. Purification by flash chromatograph on Iatrobeads neutral $(\mathrm{pH} 7)$ silica gel $\left(2.5 \% \mathrm{Et}_{2} \mathrm{O}\right.$ in hexanes) yielded $100 \mathrm{mg}(80 \%)$ of the title compound as a colorless oil. Separating the enantiomers by chiral HPLC (Daicel Chiracel ${ }^{\mathrm{TM}} \mathrm{OD}-\mathrm{H}$ column, flow rate $0.8 \mathrm{ml} / \mathrm{min}, 0.8 \% i$-PrOH, 99.2\% hexanes, $\mathrm{T}_{\mathrm{r}} 12.7(2 R, 3 S)$ and $18.3(2 S, 3 R)$ provided the enantiomer ratio: $(2 R, 3 S):(2 S, 3 R)=3.6$ : $96.4\left(93 \%\right.$ ee). $\quad[\alpha]_{\mathrm{D}}=-19.1\left(\mathrm{c} 1.02, \mathrm{CHCl}_{3}\right) ;{ }^{1} \mathrm{H} \mathrm{NMR}\left(300 \mathrm{MHz}, \mathrm{CDCl}_{3}\right): \delta 9.87(\mathrm{~d}, \mathrm{~J}=3.9 \mathrm{~Hz}, 1 \mathrm{H})$, 7.30-7.48 (m, 5H), $6.53(\mathrm{~d}, \mathrm{~J}=15.6 \mathrm{~Hz}, 1 \mathrm{H}), 6.33(\mathrm{dd}, \mathrm{J}=15.6,10.7 \mathrm{~Hz}, 1 \mathrm{H}), 2.90$ (qdd, J = 7.0, 3.9, $2.6 \mathrm{~Hz}, 1 \mathrm{H}), 2.15(\mathrm{dd}, \mathrm{J}=10.7,2.6 \mathrm{~Hz}, 1 \mathrm{H}), 1.22(\mathrm{~d}, \mathrm{~J}=7.0 \mathrm{~Hz}, 3 \mathrm{H}), 1.09(\mathrm{~s}, 9 \mathrm{H}) ;{ }^{13} \mathrm{C} \mathrm{NMR}(75 \mathrm{MHz}$, $\mathrm{CDCl}_{3}$ ): $\delta 205.4,137.2,133.8,128.6,127.4,127.2,126.3,59.2,47.2,34.2,28.6,15.6$ ppm; MS (EI, 70 eV): $m / z 215\left(\mathrm{M}^{+}-\mathrm{Me}\right), 188,173,147,105,77$; HRMS $m / z$ calcd for $\mathrm{C}_{15} \mathrm{H}_{19} \mathrm{O}\left(\mathrm{M}^{+}-\mathrm{Me}\right): 215.1436$; found 215.1439 .

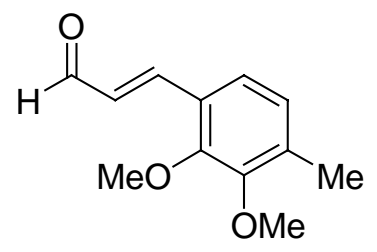

(E)-3-(2,3-Dimethoxy-4-methylphenyl)acrylaldehyde (14): ${ }^{6}$ Solution of $n$-butyllithium (1.6 $\mathrm{M}$ in hexanes, $20 \mathrm{ml}, 32 \mathrm{mmol})$ was added to a $-78{ }^{\circ} \mathrm{C}$ solution of diisopropylamine $(4.5 \mathrm{ml}, 32 \mathrm{mmol})$ in $100 \mathrm{ml}$ THF. The solution was stirred for $10 \mathrm{~min}$ then $(E)$ - $N$-ethylidene-2-methylpropan-2-amine $(2.1 \mathrm{ml}$, $16 \mathrm{mmol}$ ) was added and the reaction was stirred for $30 \mathrm{~min}$. Diethyl chlorophosphate $(2.3 \mathrm{ml}, 16 \mathrm{mmol})$ was added and the solution was stirred at $-78{ }^{\circ} \mathrm{C}$ for $2 \mathrm{~h}$

[6] For synthesis of $\alpha, \beta$-unsaturated aldehyde, see Mann, S.; Carillon, S.; Breyne, O.; Marquet, A. Chem. Eur. J. 2002, 8, 439. 
whereupon the reactions was allowed to warm to $-10{ }^{\circ} \mathrm{C}$ over $3 \mathrm{~h}$. The resulting solution was recooled to $-78{ }^{\circ} \mathrm{C}$ and a solution of 2,3-dimethoxy-4-methylbenzaldehyde $(1.93 \mathrm{~g}, 10.7 \mathrm{mmol})^{7}$ in 10 $\mathrm{ml}$ of THF was added and the reaction was allowed to warm slowly to ambient temperature overnight. A mixture of oxalic acid $(2.88 \mathrm{~g}, 32 \mathrm{mmol})$ in $100 \mathrm{ml}$ water and $100 \mathrm{ml}$ benzene was added and the resulting mixture was stirred $3 \mathrm{~h}$. The resulting mixture was extracted with ethyl ether (2 X $100 \mathrm{ml})$ and the combined organic portions wee washed successively with $5 \%$ oxalic acid, saturated $\mathrm{NaHCO}_{3}$ and brine. The extracts were concentrated and the crude product mixture was purified by column chromatography $\left(\mathrm{SiO}_{2}, 15 \%\right.$ ethyl acetate in hexanes) to afford $1.44 \mathrm{~g}(65 \%)$ of the title compound as a yellow solid. ${ }^{1} \mathrm{H}$ NMR $\left(300 \mathrm{MHz}, \mathrm{CDCl}_{3}\right): \delta 9.70(\mathrm{~d}, \mathrm{~J}=7.8 \mathrm{~Hz}, 1 \mathrm{H}), 7.77(\mathrm{~d}, \mathrm{~J}=16.1 \mathrm{~Hz}, 1 \mathrm{H}), 7.24$ $(\mathrm{d}, \mathrm{J}=8.0 \mathrm{~Hz}, 1 \mathrm{H}), 6.96(\mathrm{~d}, \mathrm{~J}=8.0 \mathrm{~Hz}, 1 \mathrm{H}), 6.72(\mathrm{dd}, \mathrm{J}=16.1,7.8 \mathrm{~Hz}, 1 \mathrm{H}), 3.92(\mathrm{~s}, 3 \mathrm{H}), 3.85(\mathrm{~s}, 3 \mathrm{H})$, 2.30 (s, 3H) ppm; $\left.{ }^{13} \mathrm{C} \mathrm{NMR} \mathrm{(75} \mathrm{MHz,} \mathrm{CDCl}_{3}\right): \delta 193.9,152.2,151.5,141.4,136.4,128.5,126.1,126.0$, 122.3, 60.9, 59.8, 16.0 ppm; IR (KBr): 2937, 2813, 2738, 1671, 1490, 1409, 1270, 1176, 1075, 1022, 822, 787, $768 \mathrm{~cm}^{-1}$; MS (EI, $\left.70 \mathrm{eV}\right): \mathrm{m} / z 206\left(\mathrm{M}^{+}\right), 191,175,160,147,135,91$; HRMS $m / z$ calcd for $\mathrm{C}_{12} \mathrm{H}_{14} \mathrm{O}_{3}\left(\mathrm{M}^{+}\right)$: 206.0943; found 206.0936.

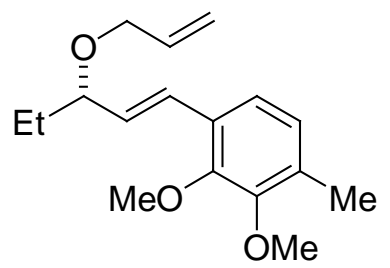

(S,E)-1-(3-(Allyloxy)pent-1-enyl)-2,3-dimethoxy-4-methylbenzene

(15):

General Procedure A was applied to

(E)-3-(2,3-dimethoxy-4-methylphenyl)acrylaldehyde (14, $1.53 \mathrm{~g}, 7.4 \mathrm{mmol})$. The crude product mixture was purified by column chromatography on silica gel (2\% ethyl acetate in hexanes) to provide $1.86 \mathrm{~g}(90 \%)$ of the title compound as light yellow oil. $[\alpha]_{\mathrm{D}}=-53.7\left(\mathrm{c} 10.4, \mathrm{CHCl}_{3}\right) ;{ }^{1} \mathrm{H} \mathrm{NMR}\left(300 \mathrm{MHz}, \mathrm{CDCl}_{3}\right): \delta 7.17(\mathrm{~d}$, $\mathrm{J}=8.0 \mathrm{~Hz}, 1 \mathrm{H}), 6.89(\mathrm{~d}, \mathrm{~J}=8.0 \mathrm{~Hz}, 1 \mathrm{H}), 6.80(\mathrm{~d}, \mathrm{~J}=16.1 \mathrm{~Hz}, 1 \mathrm{H}), 6.06(\mathrm{dd}, \mathrm{J}=16.1,8.1 \mathrm{~Hz}, 1 \mathrm{H})$, 5.90-6.02 (m, 1H), $5.30(\mathrm{~d}, \mathrm{~J}=17.2 \mathrm{~Hz}, 1 \mathrm{H}), 5.19(\mathrm{~d}, \mathrm{~J}=10.4 \mathrm{~Hz}, 1 \mathrm{H}), 4.13(\mathrm{dd}, \mathrm{J}=12.8,5.1 \mathrm{~Hz}, 1 \mathrm{H})$, $3.92(\mathrm{dd}, \mathrm{J}=12.8,5.9 \mathrm{~Hz}, 1 \mathrm{H}), 3.91(\mathrm{q}, \mathrm{J}=7.0 \mathrm{~Hz}, 1 \mathrm{H}), 3.86(\mathrm{~s}, 6 \mathrm{H}), 2.28(\mathrm{~s}, 3 \mathrm{H}), 1.59-1.82(\mathrm{~m}, 2 \mathrm{H})$, $0.98(\mathrm{t}, \mathrm{J}=7.4 \mathrm{~Hz}, 3 \mathrm{H}) \mathrm{ppm} ;{ }^{13} \mathrm{C} \mathrm{NMR}\left(75 \mathrm{MHz}, \mathrm{CDCl}_{3}\right): \delta 151.6,150.6,135.2,131.7,130.8,128.9$, 126.5, 125.7, 120.9, 116.4, 81.9, 69.1, 60.6, 60.0, 28.6, 15.7, 9.8 ppm; IR (liquid film): 2963, 2933, 2859, 1460, 1407, 1277, 1074, 1029, 916, 805, $734 \mathrm{~cm}^{-1}$; MS (EI, $\left.70 \mathrm{eV}\right): \mathrm{m} / \mathrm{z} 276\left(\mathrm{M}^{+}\right), 247,235,219$, 205, 191, 175, 165; HRMS m/z calcd for $\mathrm{C}_{17} \mathrm{H}_{24} \mathrm{O}_{3}\left(\mathrm{M}^{+}\right)$276.1725; found 276.1722 .

The enantiomeric excess of $\mathbf{1 5}$ was determined by quenching the diethylzinc addition and subjecting the resulting allylic alcohol ((S,E)-1-(2,3-dimethoxy-4-methylphenyl)pent-1-en-3-ol) to column chromatography $\left(\mathrm{SiO}_{2}, 16 \%\right.$ EtOAc in hexanes) and chiral HPLC analysis. $[\alpha]_{\mathrm{D}}=-2.6(\mathrm{c}$ 6.0, $\left.\mathrm{CHCl}_{3}\right) ;{ }^{1} \mathrm{H}$ NMR $\left(300 \mathrm{MHz}, \mathrm{CDCl}_{3}\right): \delta 7.12(\mathrm{~d}, \mathrm{~J}=8.0 \mathrm{~Hz}, 1 \mathrm{H}), 6.87(\mathrm{~d}, \mathrm{~J}=8.0 \mathrm{~Hz}, 1 \mathrm{H}), 6.81(\mathrm{~d}$, $\mathrm{J}=16.1 \mathrm{~Hz}, 1 \mathrm{H}), 6.19(\mathrm{dd}, \mathrm{J}=16.1,7.0 \mathrm{~Hz}, 1 \mathrm{H}), 4.21(\mathrm{q}, \mathrm{J}=6.5 \mathrm{~Hz}, 1 \mathrm{H}), 3.84(\mathrm{~s}, 3 \mathrm{H}), 3.83(\mathrm{~s}, 3 \mathrm{H})$, $2.25(\mathrm{~s}, 3 \mathrm{H}), 1.61-1.76(\mathrm{~m}, 2 \mathrm{H}), 0.97(\mathrm{t}, \mathrm{J}=7.4 \mathrm{~Hz}, 3 \mathrm{H}) \mathrm{ppm} ;{ }^{13} \mathrm{C} \mathrm{NMR}\left(75 \mathrm{MHz}, \mathrm{CDCl}_{3}\right): \delta 151.50$, 150.56, 132.77, 131.64, 128.97, 125.73, 124.66, 120.95, 74.65, 60.64, 60.03, 30.13, 15.76, 9.71 ppm; IR (liquid film): 3401, 2963, 2933, 1489, 1406, 1278, 1073, 1028, 973, $912 \mathrm{~cm}^{-1}$; MS (EI, $70 \mathrm{eV}$ ): $\mathrm{m} / \mathrm{z}$ $236\left(\mathrm{M}^{+}\right), 207,179,165,152$; HRMS $\mathrm{m} / z$ calcd for $\mathrm{C}_{14} \mathrm{H}_{20} \mathrm{O}_{3}\left(\mathrm{M}^{+}\right)$236.1412; found 236.1410. Separating the enantiomers by chiral HPLC [Daicel Chiracel ${ }^{\mathrm{TM}}$ OD-H column, flow rate $1.0 \mathrm{ml} / \mathrm{min}, 5$ $\% i$-PrOH, $95 \%$ hexanes, $\mathrm{T}_{\mathrm{r}} 8.84(R)$ and $9.76(S)$ ] provided the enantiomer ratio: $\mathbf{1 5}^{\prime}(R): \mathbf{1 5}^{\prime}(S)=5.2$ :

[7] Comins, D. L.; Brown, J. D. J. Org. Chem. 1984, 49, 1078. 
93.7 (90\% ee).<smiles>CC/C=C/[C@H](c1ccc(C)c(OC)c1OC)C(C)CO</smiles>

(2S,3R,E)-3-(2,3-Dimethoxy-4-methylphenyl)-2-methylhept-4-en-1-ol (16): General Procedure $\mathbf{C}$ was followed employing $1.93 \mathrm{~g}$ of di(allyl) ether 15 (7.0 $\mathrm{mmol}$ ) in 1,2-dichloroethane solvent and $30 \mathrm{~min}$ isomerization reaction time. Triphenylphsophine (55 $\mathrm{mg}, 0.21 \mathrm{mmol}$ ) was added and the resulting solution was heated at reflux for $12 \mathrm{~h} ; 2 S, 3 R: 2 R, 3 R=94: 6$. The reaction was allowed to cool to ambient temperature then it was cooled to $-78{ }^{\circ} \mathrm{C}$ and ${ }^{i} \mathrm{Bu}_{2} \mathrm{AlH}(1.0 \mathrm{M}$ in hexanes, $10.5 \mathrm{ml}, 10.5 \mathrm{mmol})$ was added dropwise. The reaction was stirred $30 \mathrm{~min}$ at $-78{ }^{\circ} \mathrm{C}$ then warmed to ambient temperature whereupon $30 \mathrm{~mL}$ of a saturated solution of sodium tartrate $30 \mathrm{ml}$ was added. The resulting mixture was extracted with ethyl ether $(3 \times 30 \mathrm{ml})$ and the combined extracts were concentrated and the crude product mixture was purified by flash chromatograph on silica gel (16\% ethyl acetate in hexanes) to yield $1.55 \mathrm{~g}(80 \%)$ of the title compound as colorless oil. $[\alpha]_{\mathrm{D}}=-9.9\left(\mathrm{c} 11.9, \mathrm{CHCl}_{3}\right) ;{ }^{1} \mathrm{H} \mathrm{NMR}\left(300 \mathrm{MHz}, \mathrm{CDCl}_{3}\right): \delta 6.89$ $(\mathrm{d}, \mathrm{J}=8.0 \mathrm{~Hz}, 1 \mathrm{H}), 6.82(\mathrm{~d}, \mathrm{~J}=8.0 \mathrm{~Hz}, 1 \mathrm{H}), 5.70(\mathrm{dd}, \mathrm{J}=15.2,8.2 \mathrm{~Hz}, 1 \mathrm{H}), 5.60(\mathrm{dt}, \mathrm{J}=15.2,5.5 \mathrm{~Hz}$, $1 \mathrm{H}), 3.85(\mathrm{~s}, 3 \mathrm{H}), 3.83(\mathrm{~s}, 3 \mathrm{H}), 3.62-3.77(\mathrm{~m}, 2 \mathrm{H}), 3.37-3.52(\mathrm{~m}, 1 \mathrm{H}), 2.37(\mathrm{~s}, 1 \mathrm{H}), 2.25(\mathrm{~s}, 3 \mathrm{H}), 0.97(\mathrm{t}$, $\mathrm{J}=7.4 \mathrm{~Hz}, 3 \mathrm{H}), 0.73(\mathrm{t}, \mathrm{J}=6.9 \mathrm{~Hz}, 3 \mathrm{H}) \mathrm{ppm} ;{ }^{13} \mathrm{C} \mathrm{NMR}\left(75 \mathrm{MHz}, \mathrm{CDCl}_{3}\right): \delta 151.27,150.70,134.40$, 132.82, 131.38, 129.72, 125.60, 123.23, 66.57, 60.57, 59.82, 43.47, 40.28, 25.46, 15.50, 13.89, 13.60; IR (liquid film): 3444, 2962, 2933, 2874, 2246, 1461, 1408, 1277, 1066, 1027, 910, 805, $734 \mathrm{~cm}^{-1}$; MS (EI, $70 \mathrm{eV}): \mathrm{m} / z 278\left(\mathrm{M}^{+}\right), 219,204,188,175$; HRMS $\mathrm{m} / z$ calcd for $\mathrm{C}_{17} \mathrm{H}_{26} \mathrm{O}_{3}\left(\mathrm{M}^{+}\right)$278.1882; found 278.1883.

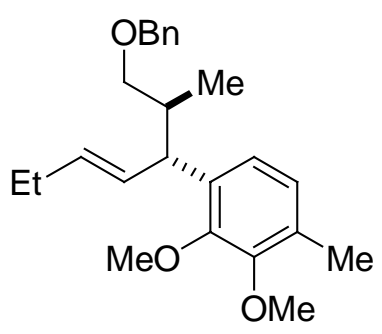

\section{1-((2S,3R,E)-1-(Benzyloxy)-2-methylhept-4-en-3-yl)-2,3-dimethoxy-4-} methylbenzene: To a suspension of sodium hydride $(360 \mathrm{mg}, 15 \mathrm{mmol})$ in $10 \mathrm{ml}$ of THF was added a solution of alcohol 16 (1.39 g, $5.0 \mathrm{mmol})$ in THF and the reaction was stirred $30 \mathrm{~min}$. Benzyl bromide $(1.71 \mathrm{~g}, 10.0 \mathrm{mmol})$ was added and the resulting mixture was warmed to $60{ }^{\circ} \mathrm{C}$ and stirred for 12 h. After cooling the reaction mixture to ambient temperature, saturated $\mathrm{NH}_{4} \mathrm{Cl}$ was added and the mixture was extracted with ethyl ether $(3 \times 30 \mathrm{ml})$.

The combined organic extracts were concentrated and the crude product mixture was purified by column chromatography on silica gel (2\% ethyl acetate in hexanes) to afford the title product $1.66 \mathrm{~g}$ (90\%) as colorless oil. $[\alpha]_{\mathrm{D}}=+16.6\left(\mathrm{c} 14.3, \mathrm{CHCl}_{3}\right) ;{ }^{1} \mathrm{H} \mathrm{NMR}\left(300 \mathrm{MHz}, \mathrm{CDCl}_{3}\right)$ : $\delta$ 7.33-7.43 (m, $5 \mathrm{H}), 6.93(\mathrm{~d}, \mathrm{~J}=8.0 \mathrm{~Hz}, 1 \mathrm{H}), 6.87(\mathrm{~d}, \mathrm{~J}=8.0 \mathrm{~Hz}, 1 \mathrm{H}), 5.61(\mathrm{dd}, \mathrm{J}=15.9,8.2 \mathrm{~Hz}, 1 \mathrm{H}), 5.53(\mathrm{dt}, \mathrm{J}=$ 15.9, $5.7 \mathrm{~Hz}, 1 \mathrm{H}), 4.60$ (d, J = 12.1 Hz, 1H), 4.54 (d, J = 12.1 Hz, 1H), 3.89 (s, 3H), 3.88 (s, 3H), 3.69 $(\mathrm{dd}, \mathrm{J}=9.5,3.6 \mathrm{~Hz}, 1 \mathrm{H}), 3.58(\mathrm{dd}, \mathrm{J}=9.5,8.5 \mathrm{~Hz}, 1 \mathrm{H}), 3.39(\mathrm{dd}, \mathrm{J}=8.8,7.4 \mathrm{~Hz}, 1 \mathrm{H}), 2.30(\mathrm{~s}, 3 \mathrm{H})$, 2.10-2.20 (m, 1H), 1.99-2.08 (m, 2H), $0.99(\mathrm{t}, \mathrm{J}=7.4 \mathrm{~Hz}, 3 \mathrm{H}), 0.91(\mathrm{~d}, \mathrm{~J}=6.9 \mathrm{~Hz}, 3 \mathrm{H}) \mathrm{ppm} ;{ }^{13} \mathrm{C} \mathrm{NMR}$ $\left(75 \mathrm{MHz}, \mathrm{CDCl}_{3}\right): \delta 151.42,150.90,138.90,135.98,132.63,131.60,129.50,128.20,127.44,127.28$, 125.54, 122.70, 74.18, 72.98, 60.34, 59.79, 45.34, 37.84, 25.49, 16.20, 15.61, 13.71 ppm; IR(liquid film): 3029, 2961, 2931, 1461, 1408, 1278, 1070, 1028, 969, 910, 734, $698 \mathrm{~cm}^{-1}$; MS (EI, $\left.70 \mathrm{eV}\right): \mathrm{m} / \mathrm{z}$ $368\left(\mathrm{M}^{+}\right), 287,277,262,247,219,188,175,91$; HRMS $m / z$ calcd for $\mathrm{C}_{24} \mathrm{H}_{32} \mathrm{O}_{3}\left(\mathrm{M}^{+}\right)$: 368.2351 ; found 368.2348 . 


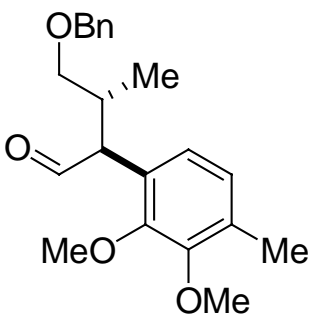

dissolved in $5 \mathrm{ml} \mathrm{MeCN}-\mathrm{H}_{2} \mathrm{O}(1: 1)$ and $\mathrm{NaIO}_{4}(214 \mathrm{mg}, 1.0 \mathrm{mmol})$ was added and the reaction was stirred $6 \mathrm{~h}$. The reaction mixture was extracted with ethyl ether and the combined organic extracts were dried and concentrated. The crude product mixture was purified by column chromatography on silica gel (5\% ethyl acetate in hexanes) to yield $267 \mathrm{mg}$ (78 \%) of the title compound as a light yellow oil; $2 S, 3 R: 2 R, 3 R=93: 7$ (assayed by $\left.{ }^{1} \mathrm{H} \mathrm{NMR}\right) . \quad[\alpha]_{\mathrm{D}}=-67 \quad\left(\mathrm{c} 6.8, \mathrm{CHCl}_{3}\right) ;{ }^{1} \mathrm{H} \mathrm{NMR}(300 \mathrm{MHz}$, $\left.\mathrm{CDCl}_{3}\right): \delta 9.71(\mathrm{~d}, \mathrm{~J}=2.8 \mathrm{~Hz}, 1 \mathrm{H}), 7.21-7.40(\mathrm{~m}, 5 \mathrm{H}), 6.90(\mathrm{~d}, \mathrm{~J}=7.9 \mathrm{~Hz}, 1 \mathrm{H}), 6.80(\mathrm{~d}, \mathrm{~J}=7.9 \mathrm{~Hz}$, $1 \mathrm{H}), 4.54(\mathrm{~d}, \mathrm{~J}=12.2 \mathrm{~Hz}, 1 \mathrm{H}), 4.48(\mathrm{~d}, \mathrm{~J}=12.2 \mathrm{~Hz}, 1 \mathrm{H}), 3.84(\mathrm{~s}, 3 \mathrm{H}), 3.81(\mathrm{~s}, 3 \mathrm{H} 0,3.77-3.81(\mathrm{~m}, 1 \mathrm{H})$, $3.52(\mathrm{dd}, \mathrm{J}=9.3,5.0 \mathrm{~Hz}, 1 \mathrm{H}), 3.39(\mathrm{dd}, \mathrm{J}=9.3,7.3 \mathrm{~Hz}, 1 \mathrm{H}), 2.66-2.77(\mathrm{~m}, 1 \mathrm{H}), 2.26(\mathrm{~s}, 3 \mathrm{H}), 0.76(\mathrm{~d}, \mathrm{~J}$ $=7.4 \mathrm{~Hz}, 3 \mathrm{H}) \mathrm{ppm} ;{ }^{13} \mathrm{C} \mathrm{NMR}\left(75 \mathrm{MHz}, \mathrm{CDCl}_{3}\right): \delta 200.5,151.7,151.5,138.2,131.8,128.3,127.6$, 127.5, 127.0, 125.7, 124.6, 74.1, 73.0, 60.5, 59.7, 56.3, 34.1, 15.7, 14.6 ppm; IR (liquid film): 2934, 2718, 1721, 1489, 1409, 1278, 1069, 1025, 916, 814, 737, $699 \mathrm{~cm}^{-1}$. MS (EI, $\left.70 \mathrm{eV}\right): \mathrm{m} / z 342\left(\mathrm{M}^{+}\right)$, 314, 206, 193, 165, 150, 135, 105; HRMS $\mathrm{m} / \mathrm{z}$ calcd for $\mathrm{C}_{20} \mathrm{H}_{26} \mathrm{O}_{3}\left(\mathrm{M}^{+}-\mathrm{CO}\right): 314.1882$; found 314.1876.

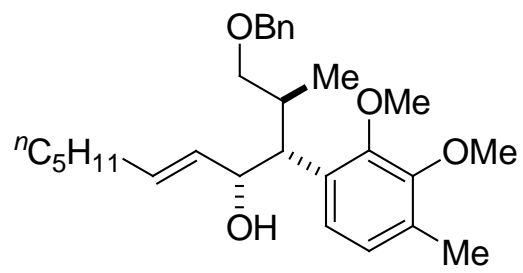

(2S,3R,4R,E)-1-(Benzyloxy)-3-(2,3-dimethoxy-4-methylphenyl)-2methylundec-5-en-4-ol (18): To a suspension of bis(cyclopentadienyl)zirconium(IV) chloride hydride (Schwartz' reagent, $260 \mathrm{mg}, 1.0 \mathrm{mmol}$ ) in $2 \mathrm{~mL}$ of $\mathrm{CH}_{2} \mathrm{Cl}_{2}$ was added 1-heptyne $(96 \mathrm{mg}, 1.0 \mathrm{mmol}$ ) and the mixture was stirred $30 \mathrm{~min}$. The reaction mixture was cooled to $-78{ }^{\circ} \mathrm{C}$ whereupon $\mathrm{Et}_{2} \mathrm{Zn}(1.0 \mathrm{M}$ in hexane, $1.0 \mathrm{ml}, 1.0 \mathrm{mmol})$ was added dropwise over $5 \mathrm{~min}$ and the reaction was stirred $30 \mathrm{~min}$ before warming to $0{ }^{\circ} \mathrm{C} .{ }^{8}$ A solution of aldehyde 17 (274 $\mathrm{mg}, 0.8 \mathrm{mmol})$ in $2 \mathrm{ml}$ toluene was added over $10 \mathrm{~min}$ and the reaction mixture was stirred $5 \mathrm{~h}$. Saturated $\mathrm{NH}_{4} \mathrm{Cl}$ was added and the resulting mixture was filtered through a plug of silica gel. The filtrate was concentrated and the resulting residue was purified by column chromatography on silica gel (20\% ethyl acetate in hexanes) to yield $236 \mathrm{mg}(67 \%)$ of the title compound as a light yellow oil. ${ }^{1} \mathrm{H}$ NMR $\left(300 \mathrm{MHz}, \mathrm{CDCl}_{3}\right): \delta 7.29-7.37(\mathrm{~m}, 5 \mathrm{H}), 6.83(\mathrm{~d}, \mathrm{~J}=8.0 \mathrm{~Hz}, 1 \mathrm{H}), 6.72(\mathrm{~d}, \mathrm{~J}=$ $8.0 \mathrm{~Hz}, 1 \mathrm{H}), 5.46(\mathrm{dt}, \mathrm{J}=15.3,6.6 \mathrm{~Hz}, 1 \mathrm{H}), 5.29(\mathrm{dd}, \mathrm{J}=15.3,7.0 \mathrm{~Hz}, 1 \mathrm{H}), 4.58(\mathrm{~d}, \mathrm{~J}=11.8 \mathrm{~Hz}, 1 \mathrm{H})$, $4.52(\mathrm{~d}, \mathrm{~J}=11.8 \mathrm{~Hz}, 1 \mathrm{H}), 4.40(\mathrm{t}, \mathrm{J}=7.2 \mathrm{~Hz}, 1 \mathrm{H}), 3.82(\mathrm{~s}, 3 \mathrm{H}), 3.79(\mathrm{~s}, 3 \mathrm{H}), 3.59(\mathrm{dd}, \mathrm{J}=9.4,5.8 \mathrm{~Hz}$, $1 \mathrm{H}), 3.45(\mathrm{dd}, \mathrm{J}=9.4,5.3 \mathrm{~Hz}, 1 \mathrm{H}), 3.19-3.25(\mathrm{~m}, 1 \mathrm{H}), 3.07(\mathrm{~s}, 1 \mathrm{H}), 2.25-2.35(\mathrm{~m}, 1 \mathrm{H}), 2.24(\mathrm{~s}, 3 \mathrm{H})$, 1.78-1.94 (m, 2H), 1.04-.129 (m, 6H), $0.86(\mathrm{t}, \mathrm{J}=6.9 \mathrm{~Hz}, 3 \mathrm{H}), 0.85(\mathrm{~d}, \mathrm{~J}=7.4 \mathrm{~Hz}, 3 \mathrm{H}) \mathrm{ppm} ;{ }^{13} \mathrm{C}$ NMR

[8] Wipf, P.; Xu, W. Tetrahedron. Lett. 1994, 35, 5197. 
(75 MHz, $\left.\mathrm{CDCl}_{3}\right): \delta 151.7,151.3,138.2,132.5,132.3,131.1,129.9,128.3,127.7,127.6,125.1,124.0$, 74.6, 74.1, 73.2, 60.2, 59.8, 35.3, 32.1, 31.1, 28.7, 22.4, 16.3, 15.6, 14.2, 13.9 ppm; IR (liquid film): 3423, 2929, 1491, 1408, 1278, 1070, 1027, 909, $734 \mathrm{~cm}^{-1}$; MS (ESI): $\mathrm{m} / z$ calcd for $\mathrm{C}_{28} \mathrm{H}_{40} \mathrm{O}_{4} \mathrm{Na}\left(\mathrm{M}^{+}+\mathrm{Na}\right)$ : 463.2824; found 463.2780.

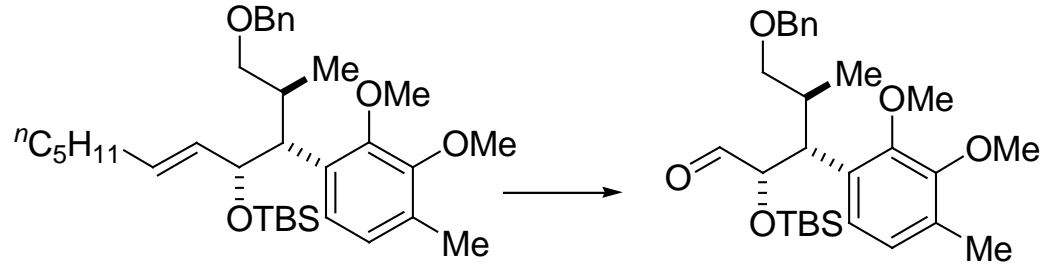

$(2 S, 3 R, 4 S)$-5-Benzyloxy)-2-(tert-buty Idimethylsilyloxy)-3-(2,3-dimethoxy4-methylphenyl)-4-methylpentanal (19): To a solution of alcohol 18 (220 $\mathrm{mg}, 0.5 \mathrm{mmol})$ in DMF (2 $\mathrm{ml})$ was added imidazole $(66 \mathrm{mg}, 1 \mathrm{mmol})$ and

TBSCl $\left(90 \mathrm{mg}, 0.6 \mathrm{mmol}\right.$ ) and the reaction was heated at $80{ }^{\circ} \mathrm{C}$ for $3 \mathrm{~h}$. The reaction mixture was cooled to ambient temperature, diluted with water, and extracted with ethyl ether. The combined organic extracts were concentrated and the resulting crude product mixture was dissolved in $20 \mathrm{ml}$ methanol and cooled to the resulting solution was cooled $-78{ }^{\circ} \mathrm{C}$. Ozone was bubbled into the solution until the blue color persisted. Dimethyl sulfide $(0.4 \mathrm{ml})$ was added and the reaction was allowed to warm to ambient temperature and stirred overnight. The solvent was evaporated and the oil was purified by column chromatography (5\% ethyl acetate in hexanes) to yield $194 \mathrm{mg}$ ( $72 \%$ for two steps) of the title compound as yellow oil. ${ }^{1} \mathrm{H} \mathrm{NMR}\left(300 \mathrm{MHz}, \mathrm{CDCl}_{3}\right): \delta 9.49$ (d, J = $1.2 \mathrm{~Hz}$, 1H), 7.42-7.23 (m, 5H), $6.89(\mathrm{~d}, \mathrm{~J}=8.0 \mathrm{~Hz}, 1 \mathrm{H}), 6.84(\mathrm{~d}, \mathrm{~J}=8.0 \mathrm{~Hz}, 1 \mathrm{H}), 4.47(\mathrm{~d}, \mathrm{~J}=11.9 \mathrm{~Hz}, 1 \mathrm{H})$, $4.39(\mathrm{~d}, \mathrm{~J}=11.9 \mathrm{~Hz}, 1 \mathrm{H}), 4.16(\mathrm{dd}, \mathrm{J}=4.7,1.2 \mathrm{~Hz}, 1 \mathrm{H}), 3.84(\mathrm{~s}, 3 \mathrm{H}), 3.80(\mathrm{~s}, 3 \mathrm{H}), 3.68(\mathrm{dd}, \mathrm{J}=9.0$, $4.6 \mathrm{~Hz}, 1 \mathrm{H}), 3.42(\mathrm{dd}, \mathrm{J}=9.2,4.6 \mathrm{~Hz}, 1 \mathrm{H}), 3.37(\mathrm{dd}, \mathrm{J}=9.2,5.5 \mathrm{~Hz}, 1 \mathrm{H}), 2.37-2.50(\mathrm{~m}, 1 \mathrm{H}), 2.48(\mathrm{~s}$, $3 \mathrm{H}), 0.92$ (s, 9H), 0.88 (d, J = $6.9 \mathrm{~Hz}, 3 \mathrm{H}),-0.01$ (s, 3H), -0.12 (s, 3H) ppm; IR(liquid film): 2930, 2857, 1736, 1462, 1408, 1279, 1254, 1159, 1071, 1027, 838, 778, $698 \mathrm{~cm}^{-1}$; MS (EI, $70 \mathrm{eV}$ ): $\mathrm{m} / \mathrm{z} 457$ ( $\left.\mathrm{M}^{+}-\mathrm{CHO}\right), 429,349$, 337321, 207; HRMS $\mathrm{m} / \mathrm{z}$ calcd for $\mathrm{C}_{27} \mathrm{H}_{41} \mathrm{O}_{4} \mathrm{Si}\left(\mathrm{M}^{+}-\mathrm{CHO}\right): 457.2774$, found: 457.2761.

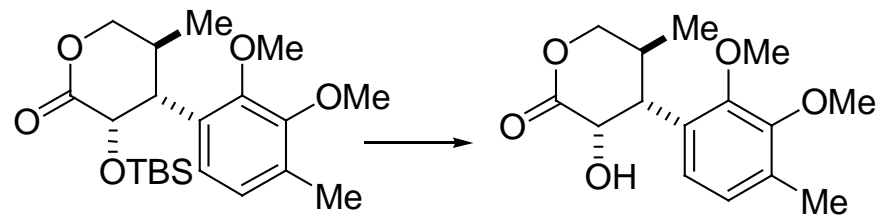

(3S,4R,5S)-4-(2,3-Dimethoxy-4-methylphenyl) -3-hydroxy-5-methyl-tetrahydropyran-2-one (13): ${ }^{9}$ A suspension of aldehyde 19 (100 mg, $0.2 \mathrm{mmol})$ and $5 \% \mathrm{Pd} / \mathrm{C}(10 \mathrm{mg})$ in ethyl acetate (10 ml) was placed under $1 \mathrm{~atm}$ of $\mathrm{H}_{2}$ (balloon) and strirred for $12 \mathrm{~h}$. The hydrogen gas was purged from the reaction flask with a stream of $\mathrm{N}_{2}$ and the reaction mixture was filtered through Celite using ethyl ether to wash the filter cake. The filtrate was concentrated and the resulting oil was dissolved in $10 \mathrm{ml}$ of $\mathrm{CH}_{2} \mathrm{Cl}_{2}$ containing 4-methylmorpholine $N$-oxide $(34 \mathrm{mg}, 0.3 \mathrm{mmol})$ and $4 \AA \mathrm{MS}(100 \mathrm{mg})$. Tetrapropylammonium perruthenate (TPAP, $3.5 \mathrm{mg}, 0.01 \mathrm{mmol}$ ) was added to the reaction mixture and the resulting mixture was stirred for $2 \mathrm{~h}$. The reaction mixture was filtered through a silica gel plug using $\mathrm{CH}_{2} \mathrm{Cl}_{2}$ as the eluent. The filtrate was concentrated to afford

[9] Luzung, M. R.; Toste, F. D. J. Am. Chem. Soc. 2003, 125, 15760. 
(3S,4R,5S)-4-(2,3-dimethoxy-4-methylphenyl)-3-tetr-butyldimethylsilyloxy-5-methyl-tetrahydropyran2 -one $(68 \mathrm{mg})$ that was used in the subsequent transformation without further purification. $[\alpha]_{\mathrm{D}}=$ $-36.5\left(\mathrm{c} 0.46, \mathrm{CHCl}_{3}\right) ;{ }^{1} \mathrm{H} \mathrm{NMR}\left(300 \mathrm{MHz}, \mathrm{CDCl}_{3}\right): \delta 6.84(\mathrm{~d}, \mathrm{~J}=7.9 \mathrm{~Hz}, 1 \mathrm{H}), 6.79(\mathrm{~d}, \mathrm{~J}=7.9 \mathrm{~Hz}$, $1 \mathrm{H}), 4.49(\mathrm{dd}, \mathrm{J}=11.1,5.1 \mathrm{~Hz}, 1 \mathrm{H}), 4.38(\mathrm{~d}, \mathrm{~J}=6.2 \mathrm{~Hz}, 1 \mathrm{H}), 4.04(\mathrm{dd}, \mathrm{J}=11.1,9.6 \mathrm{~Hz}, 1 \mathrm{H}), 3.85(\mathrm{~s}$, $3 \mathrm{H}), 3.78(\mathrm{~s}, 3 \mathrm{H}), 3.26(\mathrm{dd}, \mathrm{J}=9.6,6.2 \mathrm{~Hz}, 1 \mathrm{H}), 2.42-2.55(\mathrm{~m}, 1 \mathrm{H}), 2.25(\mathrm{~s}, 3 \mathrm{H}), 0.96(\mathrm{~d}, \mathrm{~J}=6.9 \mathrm{~Hz}$, $3 \mathrm{H}), 0.74(\mathrm{~s}, 9 \mathrm{H}), 0.00(\mathrm{~s}, 3 \mathrm{H}),-0.24(\mathrm{~s}, 3 \mathrm{H}) \mathrm{ppm}$.

The protected lactone obtained above $(38 \mathrm{mg}, 0.1 \mathrm{mmol})$ was dissolved in DMF (1 ml) and tris(dimethylamino)sulfonium difluorotrimethylsilicate (TASF, $55 \mathrm{mg}, 0.2 \mathrm{mmol})^{10}$ was added. The reaction mixture was stirred $3 \mathrm{~h}$ then diluted with ethyl ether $(10 \mathrm{ml})$ and the resulting mixture was washed with brine. The organic portion was dried, concentrated, and the resulting crude product mixture was purified by column chromatography (50\% ethyl acetate in hexanes) to afford $25 \mathrm{mg}(81 \%$ over three steps) of the title product as colorless oil. $\quad[\alpha]_{\mathrm{D}}=+42\left(\mathrm{c} 0.3, \mathrm{CHCl}_{3}\right) ;{ }^{1} \mathrm{H} \mathrm{NMR}(300 \mathrm{MHz}$, $\left.\mathrm{CDCl}_{3}\right): \delta 6.87(\mathrm{~d}, \mathrm{~J}=7.8 \mathrm{~Hz}, 1 \mathrm{H}), 6.70(\mathrm{~d}, \mathrm{~J}=7.8 \mathrm{~Hz}, 1 \mathrm{H}), 4.62(\mathrm{dd}, \mathrm{J}=10.0,5.1 \mathrm{~Hz}, 1 \mathrm{H}), 4.32(\mathrm{dd}, \mathrm{J}$ $=11.5,5.1 \mathrm{~Hz}, 1 \mathrm{H}), 4.12(\mathrm{t}, \mathrm{J}=11.5 \mathrm{~Hz}, 1 \mathrm{H}), 3.88(\mathrm{~s}, 3 \mathrm{H}), 3.80(\mathrm{~s}, 3 \mathrm{H}), 3.59(\mathrm{dd}, \mathrm{J}=9.7,7.9 \mathrm{~Hz}, 1 \mathrm{H})$, 2.27-2.32 (m, 1H), $2.25(\mathrm{~s}, 3 \mathrm{H}), 1.06(\mathrm{~d}, \mathrm{~J}=6.9 \mathrm{~Hz}, 3 \mathrm{H}) \mathrm{ppm} ;{ }^{13} \mathrm{C} \mathrm{NMR}\left(75 \mathrm{MHz}, \mathrm{CDCl}_{3}\right): \delta 175.03$, 151.47, 150.98, 131.97, 130.14, 125.47, 124.19, 77.20, 70.81, 66.72, 60.21, 59.76, 36.34, 16.40, 15.74 ppm; IR (liquid film): 3467, 2927, 1740, 1463, 1409, 1277, 1071, $1028 \mathrm{~cm}^{-1}$; MS (EI, $70 \mathrm{eV}$ ): $\mathrm{m} / \mathrm{z} 280$ $\left(\mathrm{M}^{+}\right), 251,205,165,91$; HRMS $\mathrm{m} / z$ calcd for $\mathrm{C}_{15} \mathrm{H}_{20} \mathrm{O}_{5}$ 280.1311; found 280.1311 .

Determination of Claisen adduct absolute stereochemistry. A comparison of the spectral and optical data for the calopin dimethyl ether (13) with that reported previously for this compound provides an unambiguous stereochemical proof for Claisen adduct 16. The absolute stereochemistry of the remaining Claisen adducts (7a-f and $\mathbf{9}$ ) was assigned by analogy to this determination.

[10] Scheidt, K. A.; Chen, H.; Follows, B. C.; Chemler, S. R.; Coffey, D. S.; Roush, W. R. J. Org. Chem. 1998, 63, 6436. 


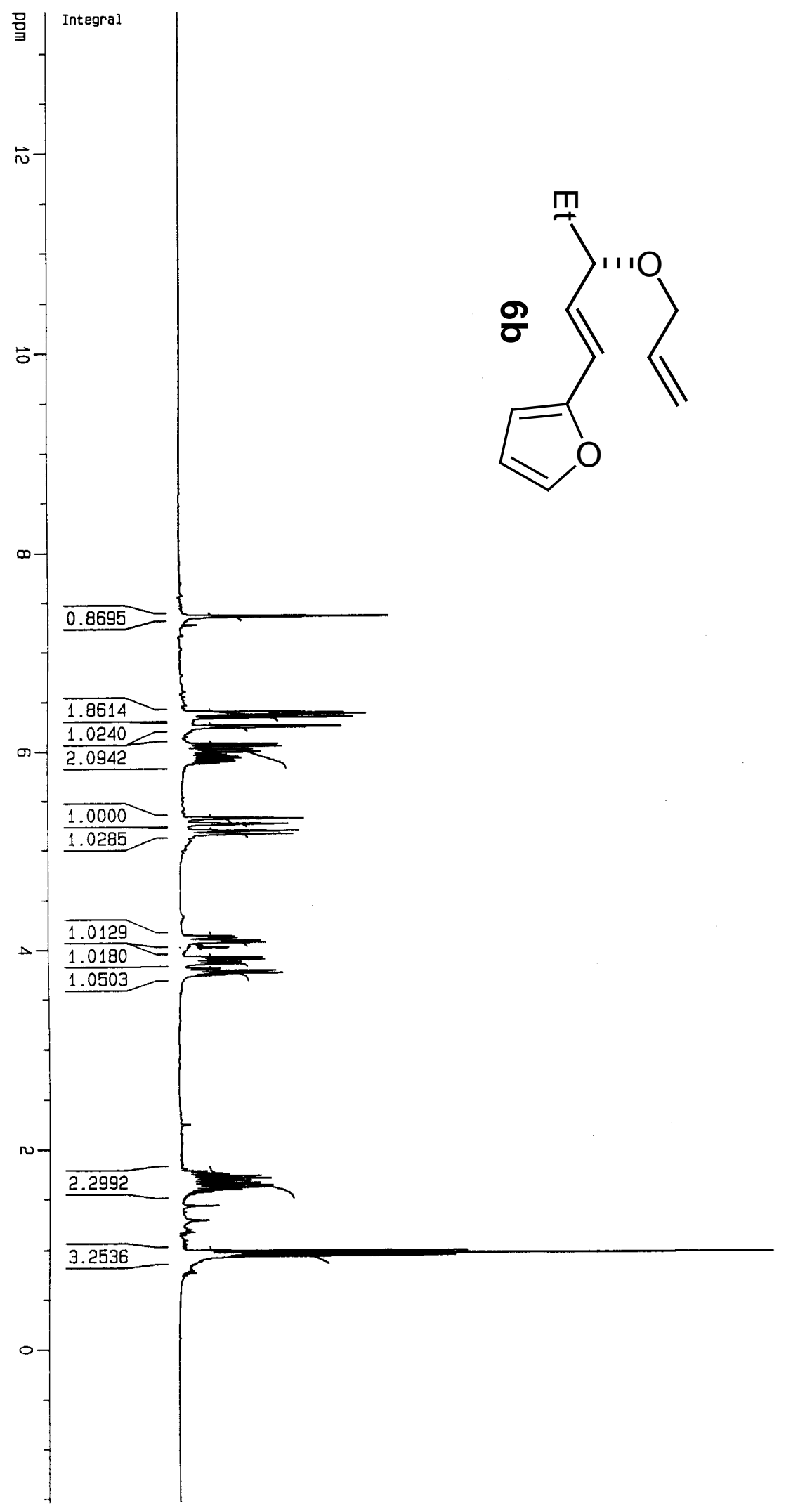

$-11.4776$

$-10.6518$

$-10.3179$

$-10.2307$

$\geq 10.2065$

$-9.8010$

$-9.2033$

$\int\left[\begin{array}{l}7.9080 \\ 7.6891\end{array}\right.$

F 7.6891

- 7.3719

$-7.3609$

- 7.3552

- 7.2707

$-6.3958$

- 6.3902

- 6.3841

$=-6.3792$

$-6.3732$

$-6.3427$

$-6.2579$

- 6.2472

$-6.0758$

$-6.0502$

$-5.3262$

$-5.3205$

ล 5.3152

$-5.2631$

$-5.1977$

$-5.1960$

ᄂ 5.1916

$7 \quad[5.1873$

$=\left[\begin{array}{l}5.1571 \\ 5.152 \theta\end{array}\right.$

L 5.1528

$-4.0743$

$-3.9239$

L 3.9040

L 3.7898

L 3.7652

1.7339

$-1.7129$

$\mathbb{M} 1.7093$

$-1.6853$

$7\lfloor-1.6621$

ㄴ 1.6375

$-1.6159$

L 1.5918

$\leftarrow 0.9856$

L 0.9611

$-0.9360$

$L 0.7625$

$[0.4742$

$-0.1111$ 

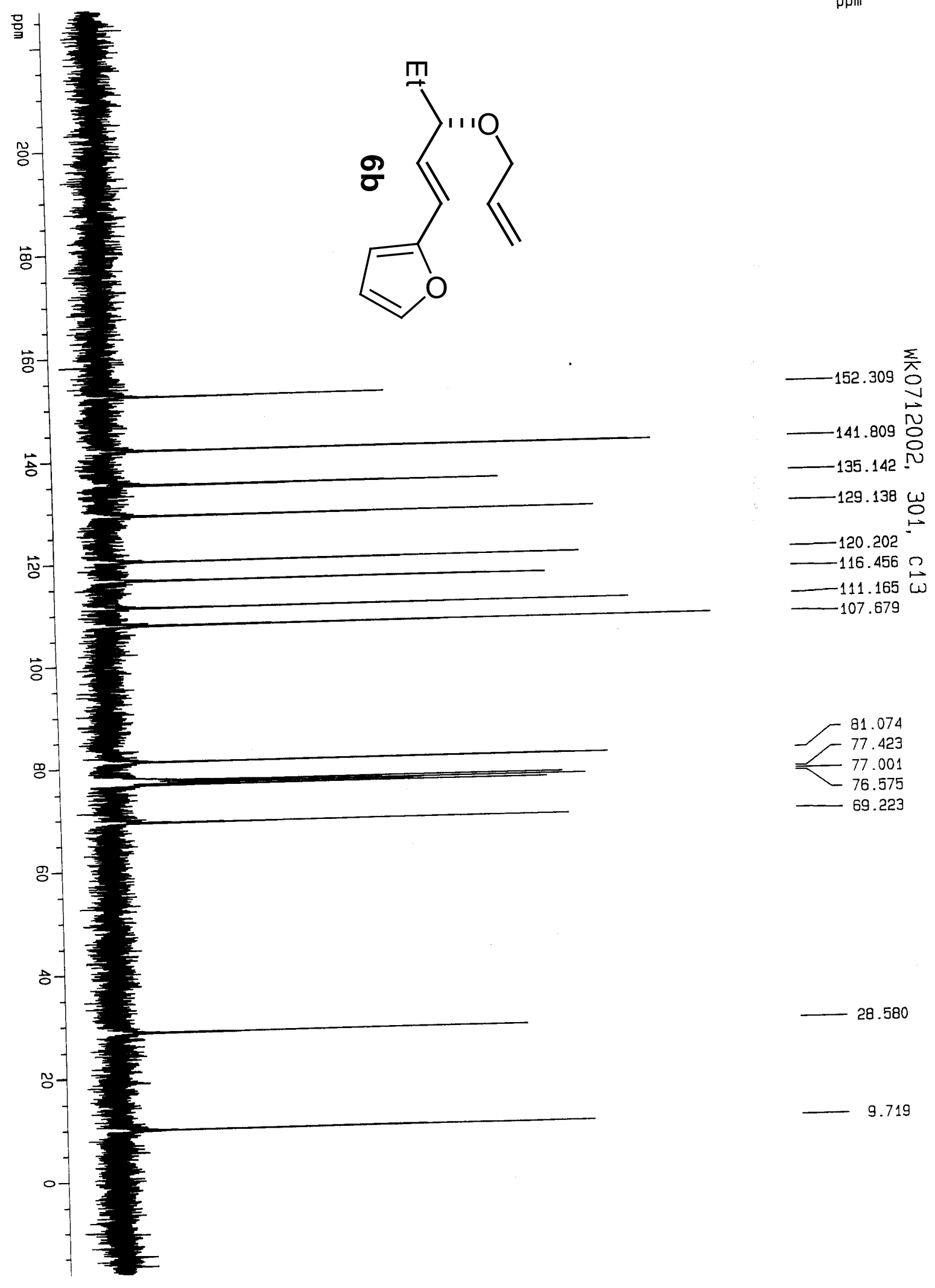

81.074

77.423

77.001

76.575

69.223

28.580

- S16 - 


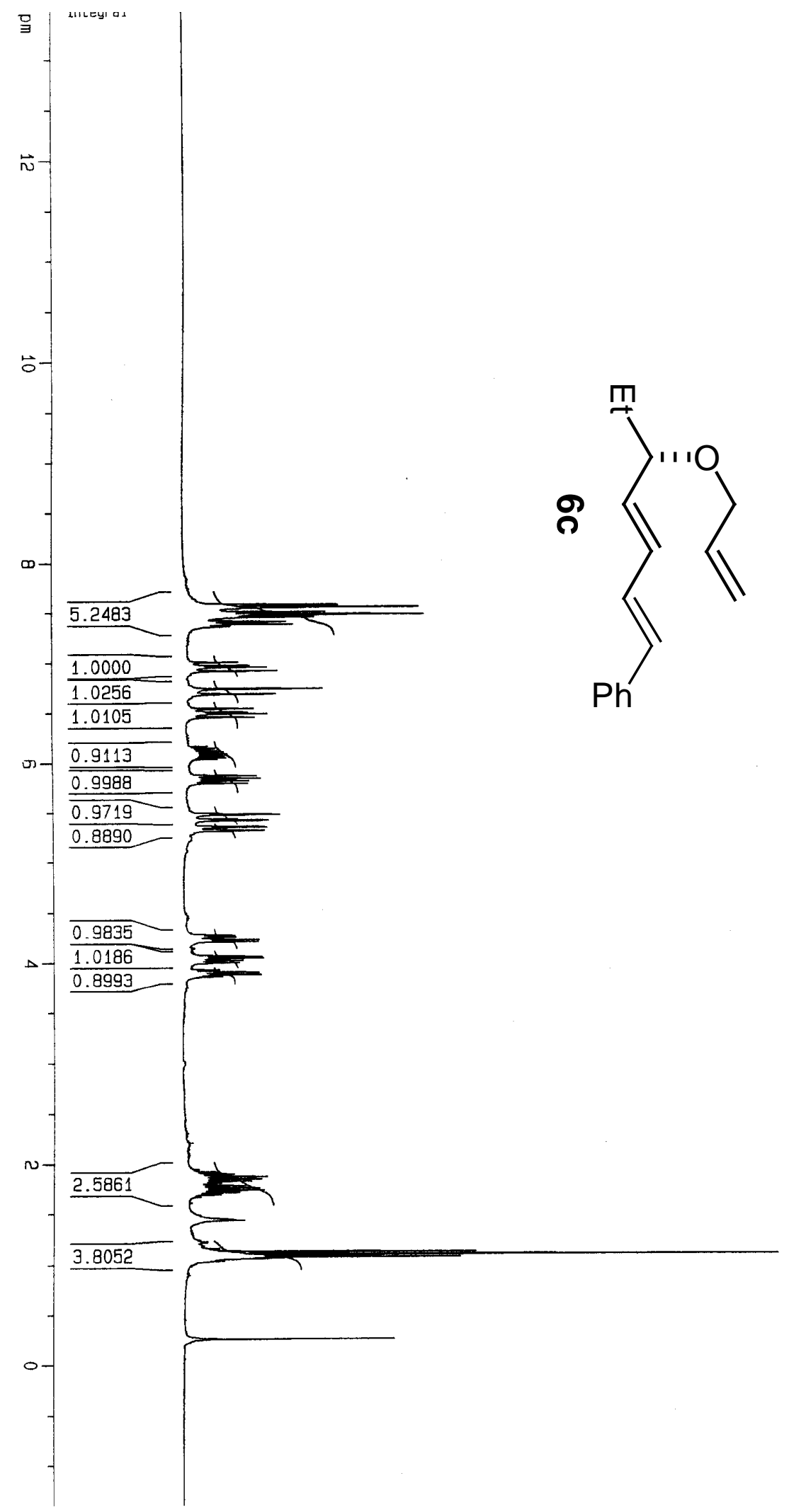

คri!

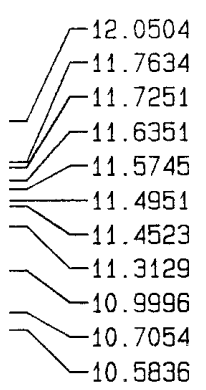

7.7452

$-7.5839$

- 7.5787

7.5552

Г 7.5527

- 7.5077

- 7.5046

$-7.4800$

$-7.4757$

$-7.4602$

$-7.4554$

] $\left[\begin{array}{l}7.4106 \\ -7.4071\end{array}\right.$

$\left[\begin{array}{l}7.4071 \\ 7.3865\end{array}\right.$

- 6.9509

$-6.9179$

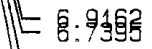

. $L 6.0073$

$\leftarrow 6.4925$

$-5.4831$

- 5.4773

$-5.4200$

] $\left[\begin{array}{l}5.3573 \\ 5.3511\end{array}\right.$

$7\lfloor-5.3511$

L 4.2285

L 4.2113

4.0641

4.0442

L 3.9058

- .8813

1.8721

1.87210
-1.8510

$-1.8474$

$-1.8262$

a -1.7795

$-1.7571$

$-1.7551$

$-1.7330$

] 1.1259

$\left[\begin{array}{l}1.1012 \\ 1.0764\end{array}\right.$

L 1.0764

$L_{1.0613}$

L 0.2703

$-0.2574$

L 0.2453 


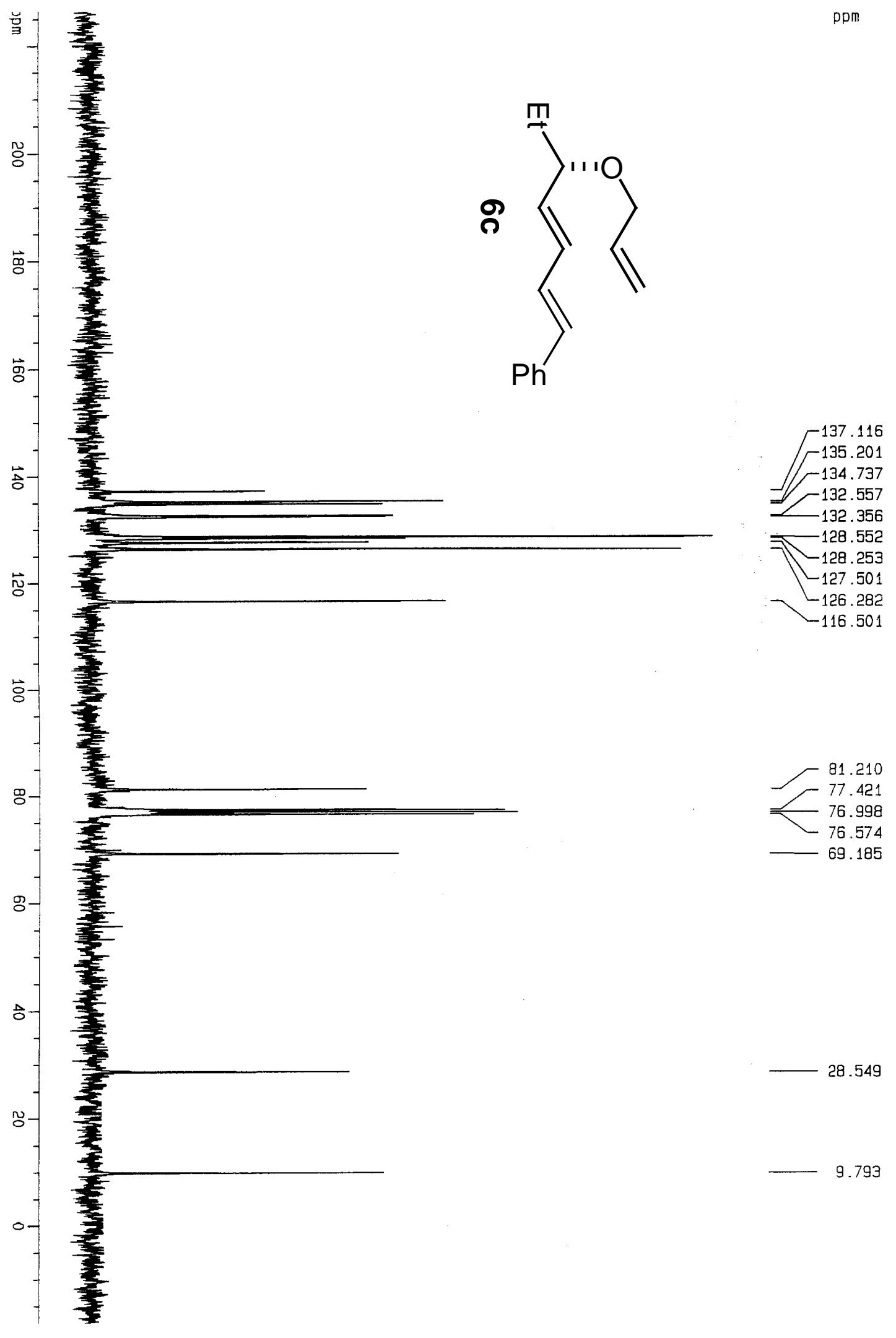




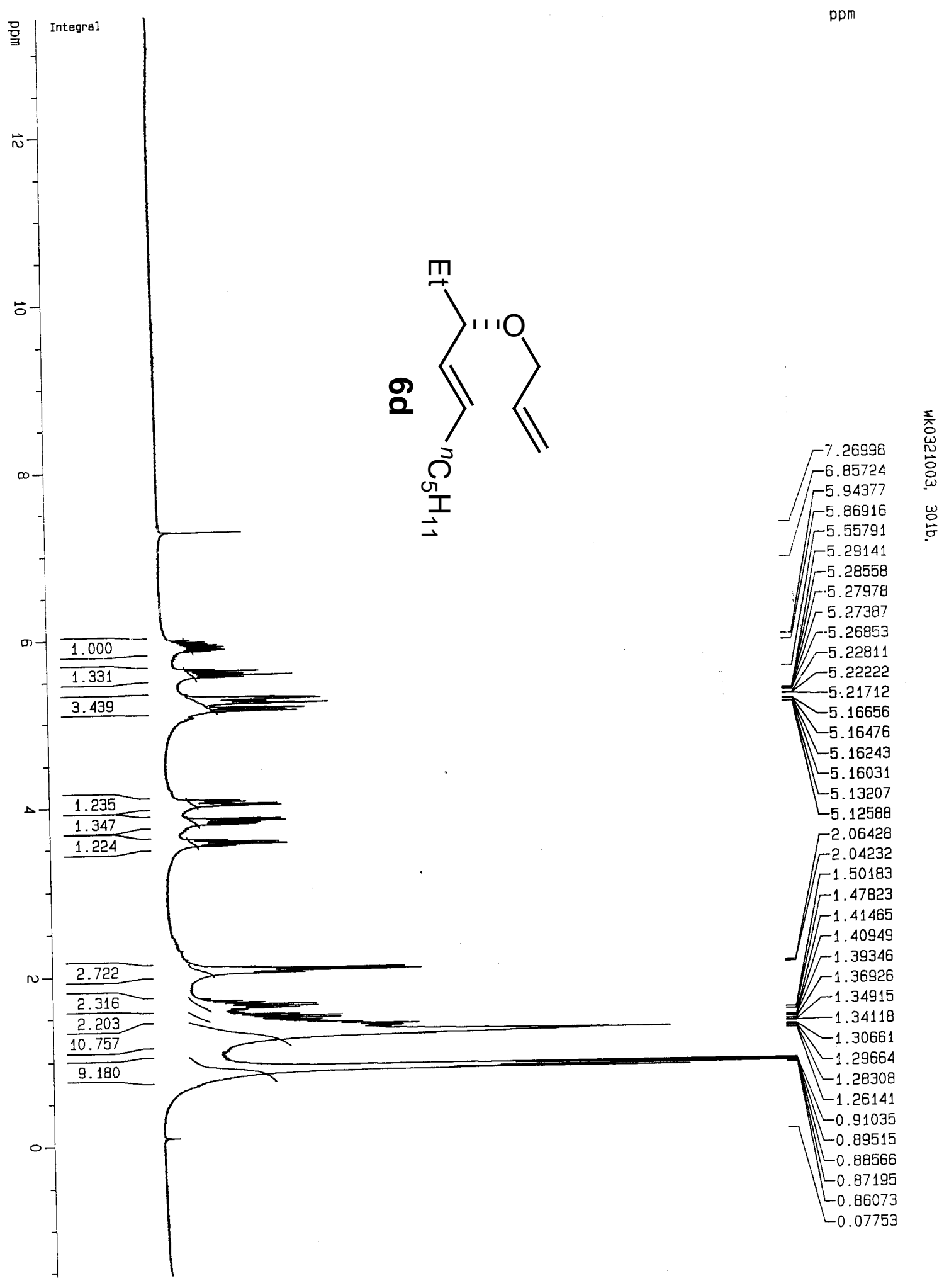




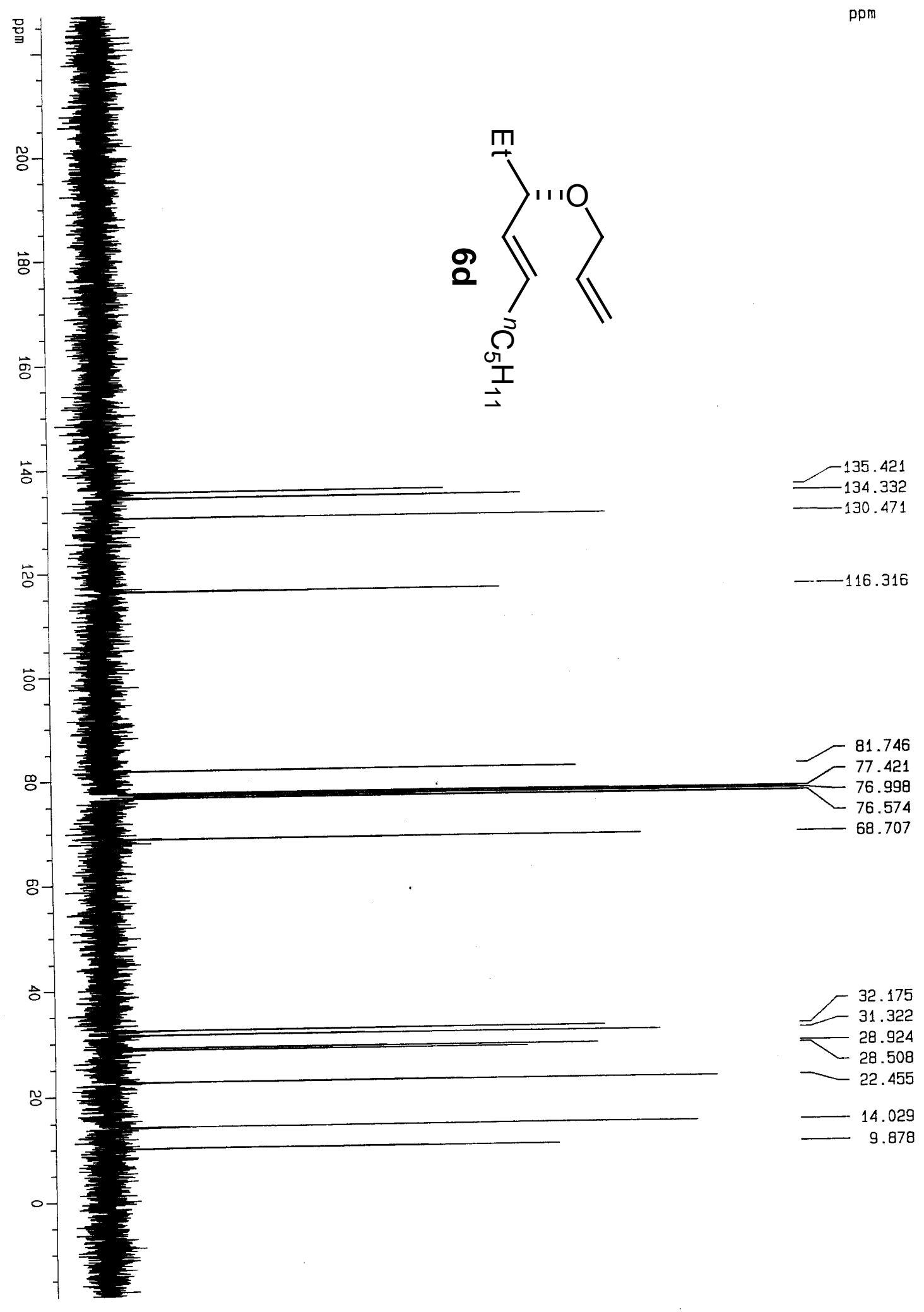




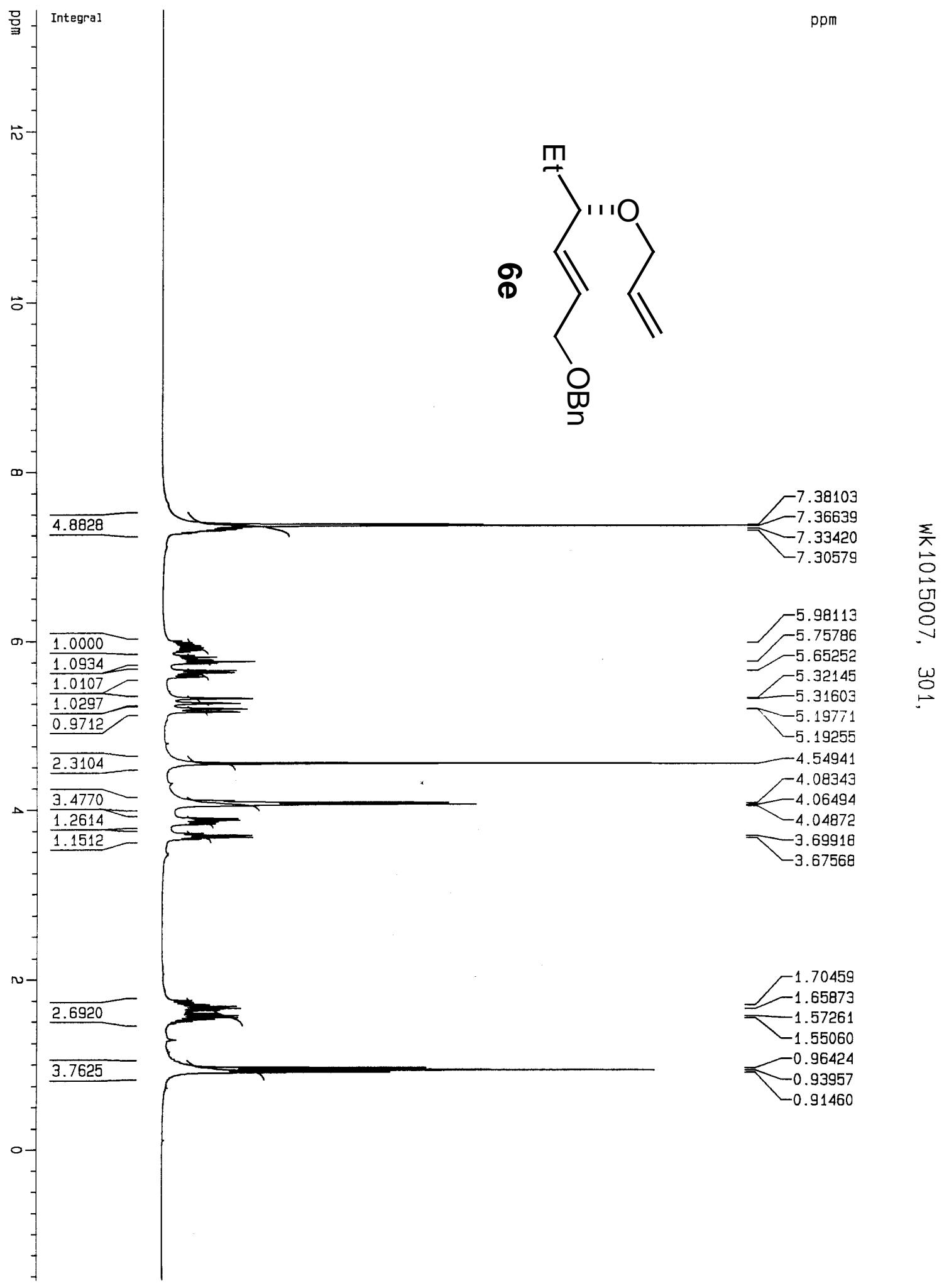




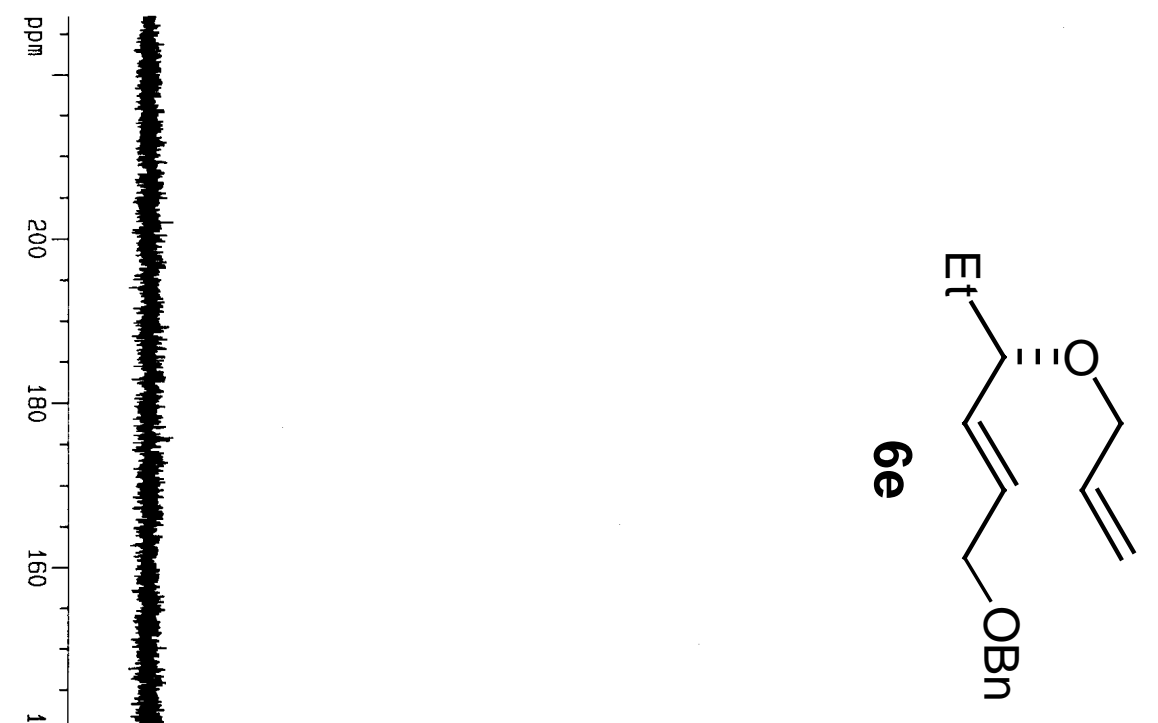

138.160

$\tau^{135.112}$

$-133.724$

$-129.241$

$-128.321$

$-128.252$

$-127.662$

$-127.592$

$-127.552$

$-116.458$

홍

\&

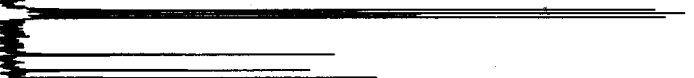

$\tau 77.422$

76.999
$=76.574$

$-71.980$

$-70.046$

$-69.128$

g-

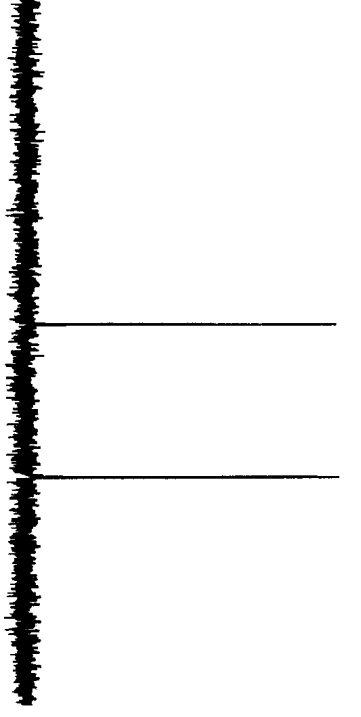

28.323

9.751 


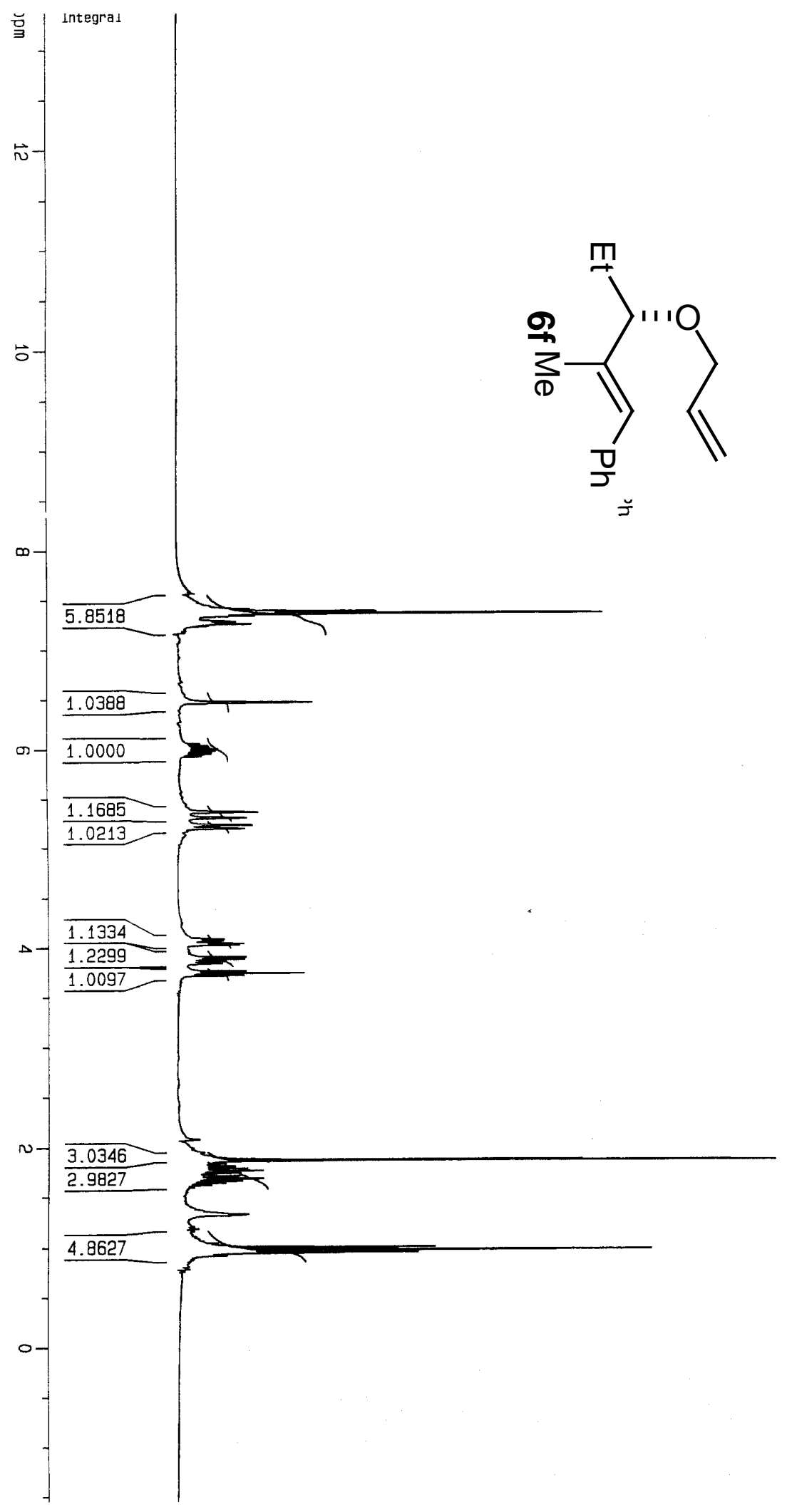

ผ)III

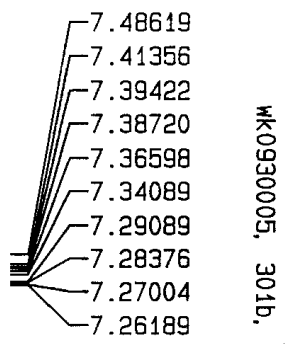

$-6.47119$

$-5.36634$

5.36088

$-5.30903$

-5.30353

$-5.23553$

D-5.23076

$-5.20108$

$-5.19630$

4.04450

$-4.02736$

$-3.90769$

$-3.88747$

$-3.76841$

$-3.74546$

$-3.72250$

1.87288

$-1.86867$

$r^{1.81332}$

$-1.79108$

$-1.76729$

$-1.74354$

$-1.71180$

-1.68743

$=\lfloor-1.66336$

$-1.32824$

$-1.31876$

$-1.01389$

$-1.00232$

$-0.97762$

$-0.95273$

$-0.94533$

$L_{0.69758}$ 

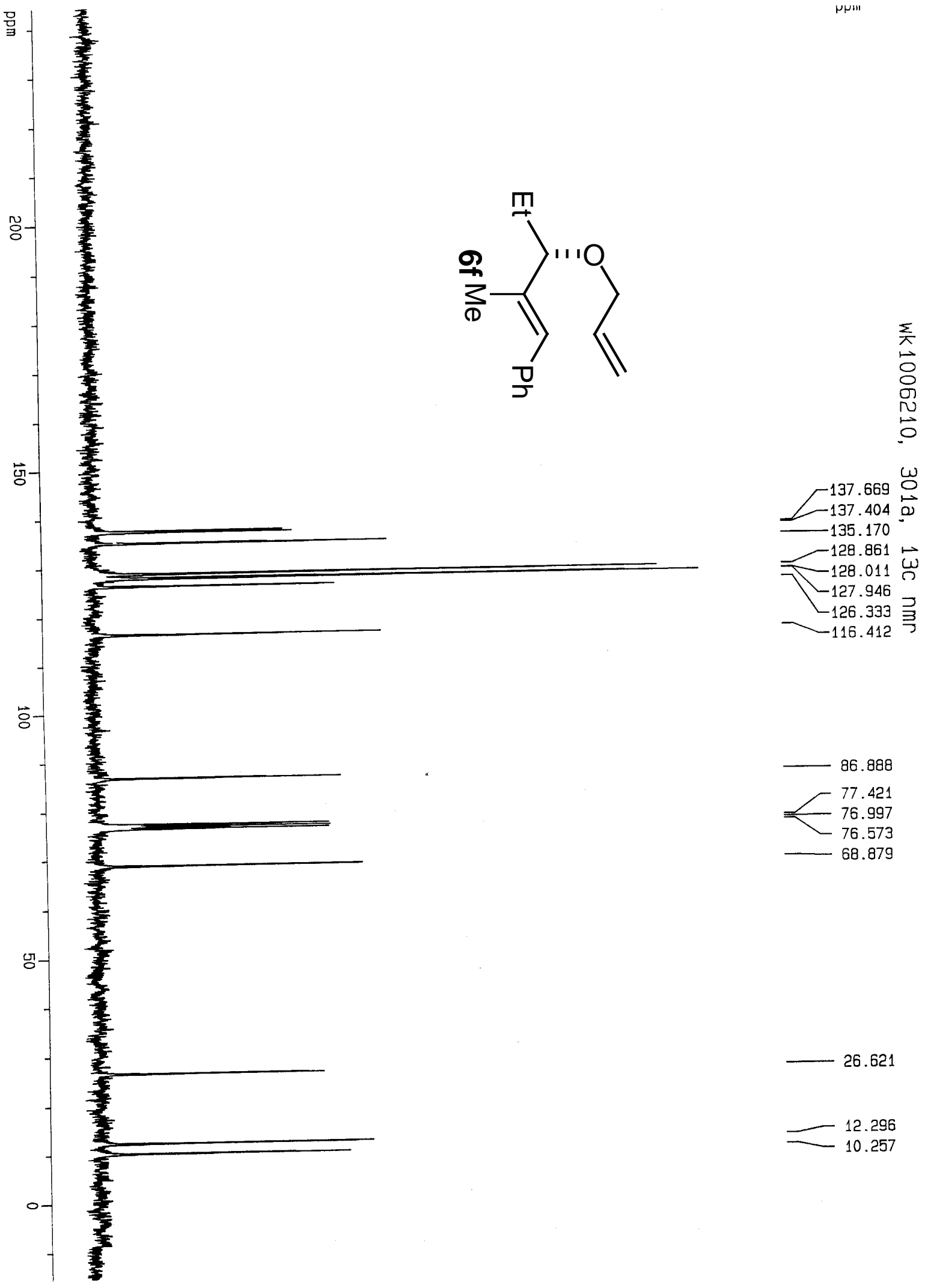

- 86.888

77.421

76.997

76.573

68.879

26.621

12. 296

$\begin{array}{r}=10.257 \\ \hline\end{array}$ 


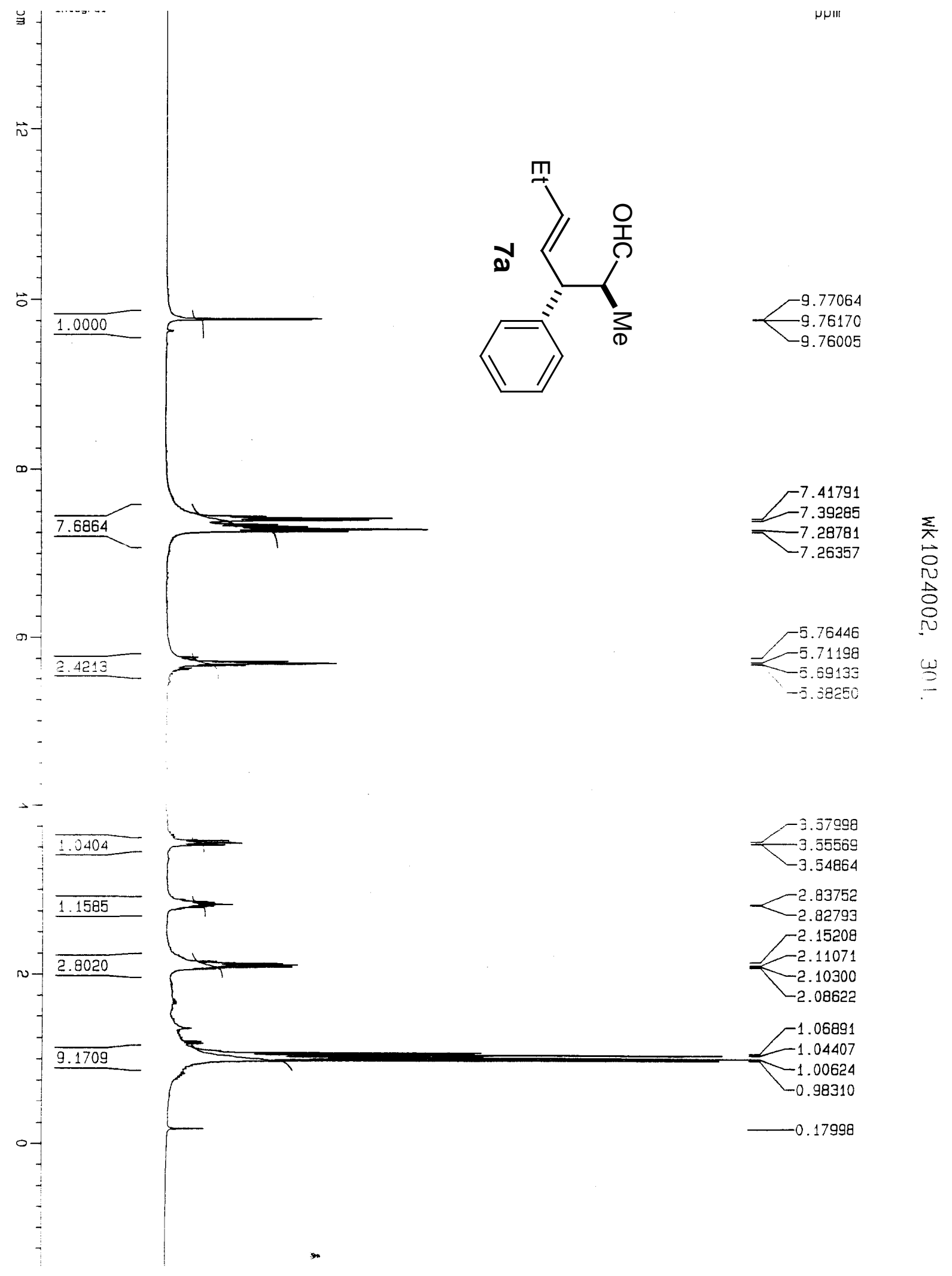




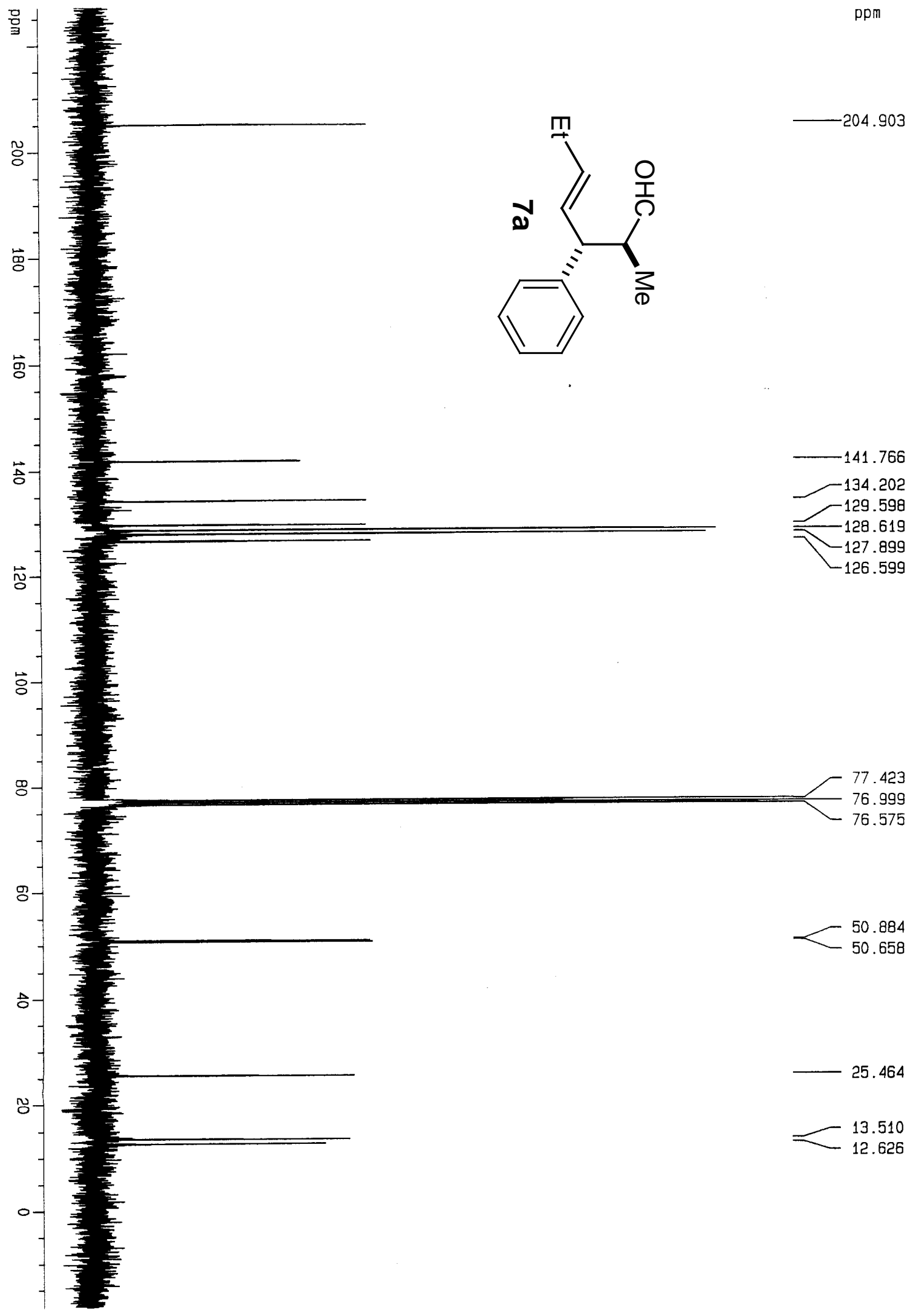




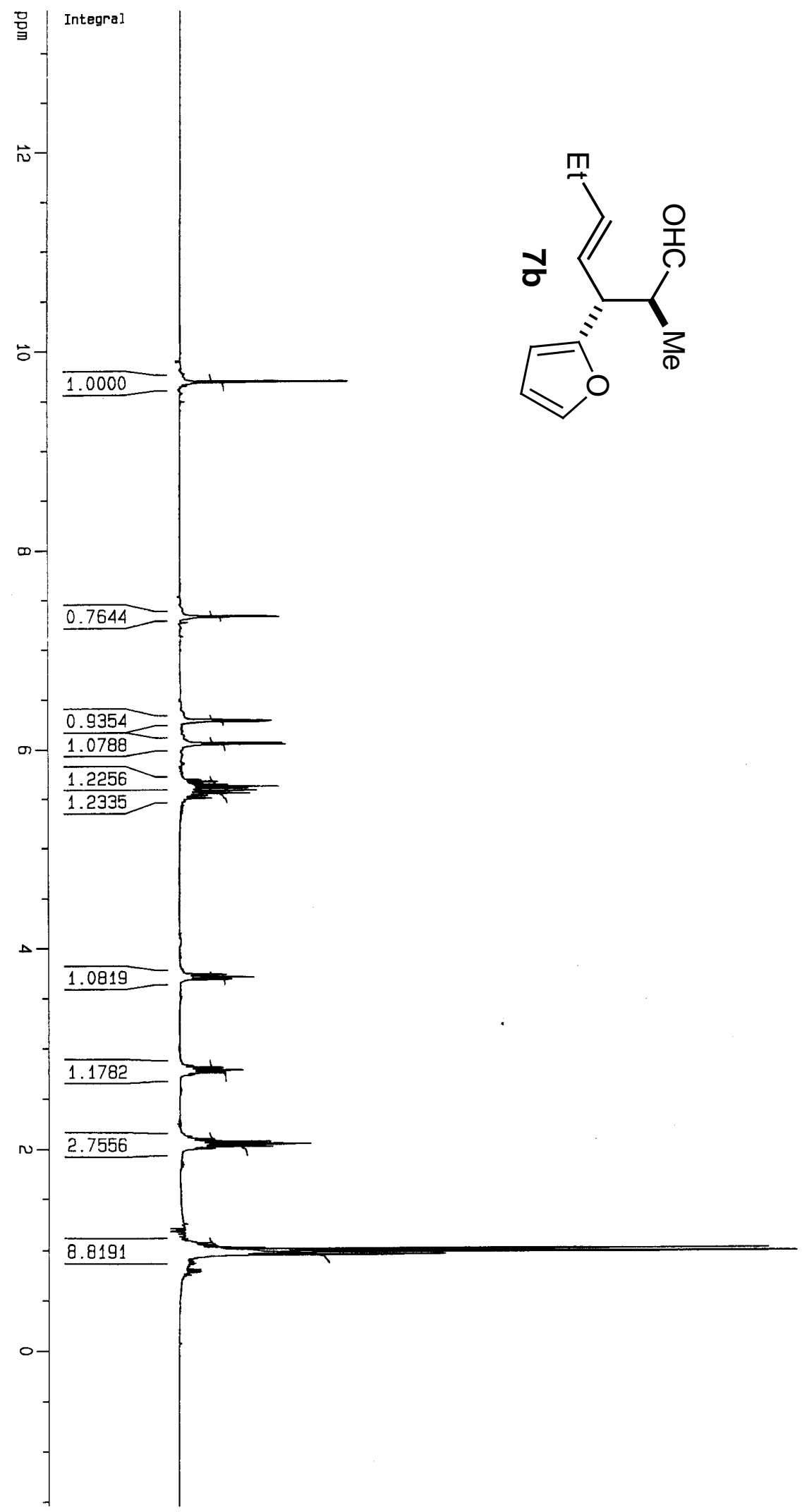

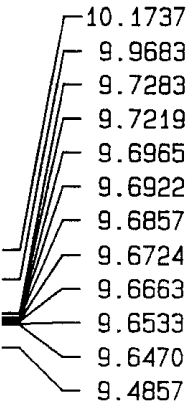

7.3374

$-7.3346$

- 7.3312

$\Gamma 7.3284$

- 6.2986

$-6.2923$

] 6.2890

- 6.2879

- 6.2826

- 6.2817

$\tau^{6.0658}$

6.0631

6.0604

$-6.0552$

- 6.0497

$-5.6447$

$-5.6251$

$-5.6062$

5.5804

5.5768

5.5530

$=3.7118$

$-3.6875$

$-2.7894$

$-2.7829$

2.0766

$-2.0742$

2.0730
-2.0521

2.0521

$-2.0313$

$-2.0276$

1.0238

1.0115

$\mathbb{- 1 . 0 0 5 7}$

$-1.0012$

0.9882

$\sqrt{-0.9807}$

0.9721

L 0.9558

L 0.9470

0.7200

$-0.4865$

0.0750 


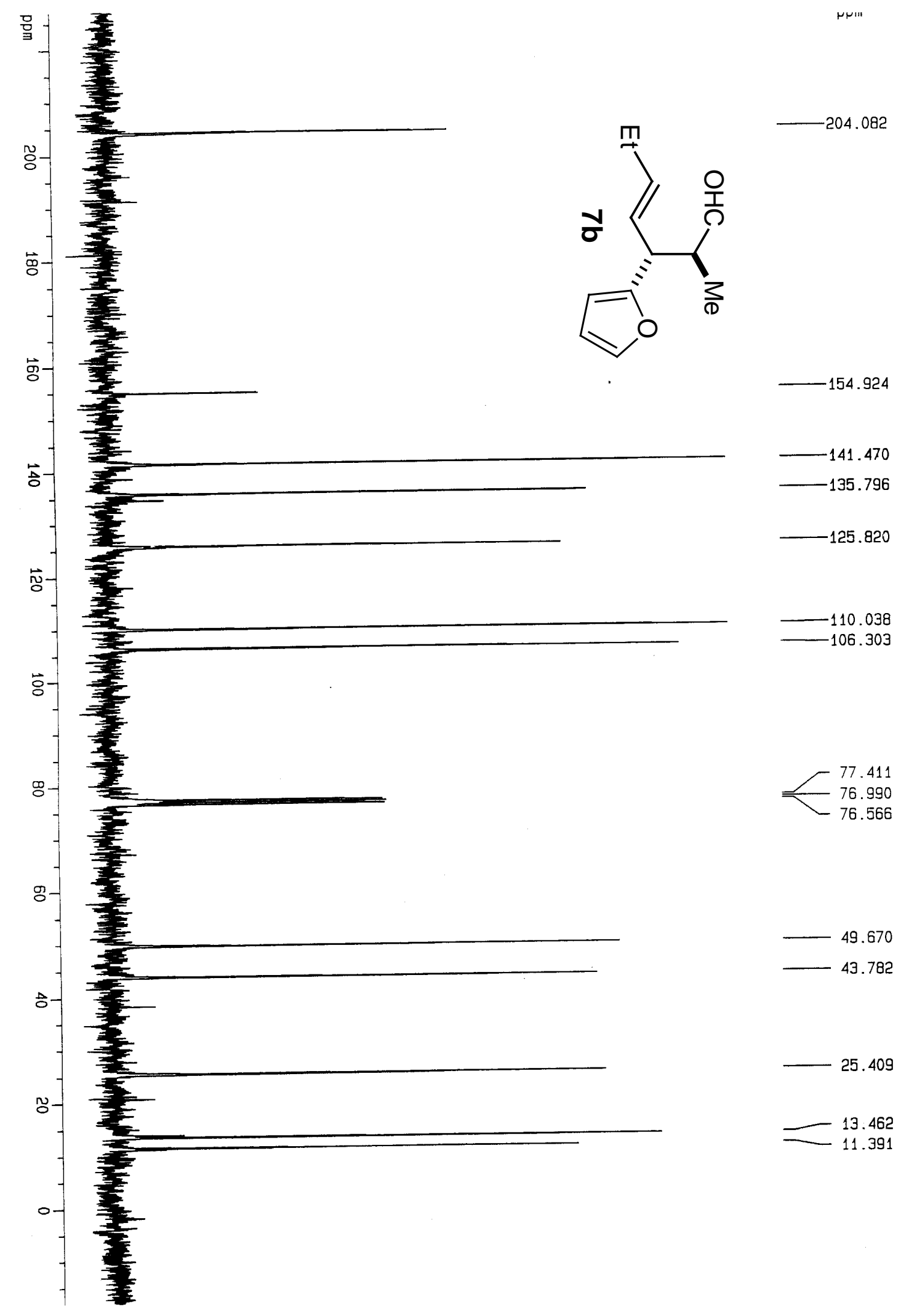




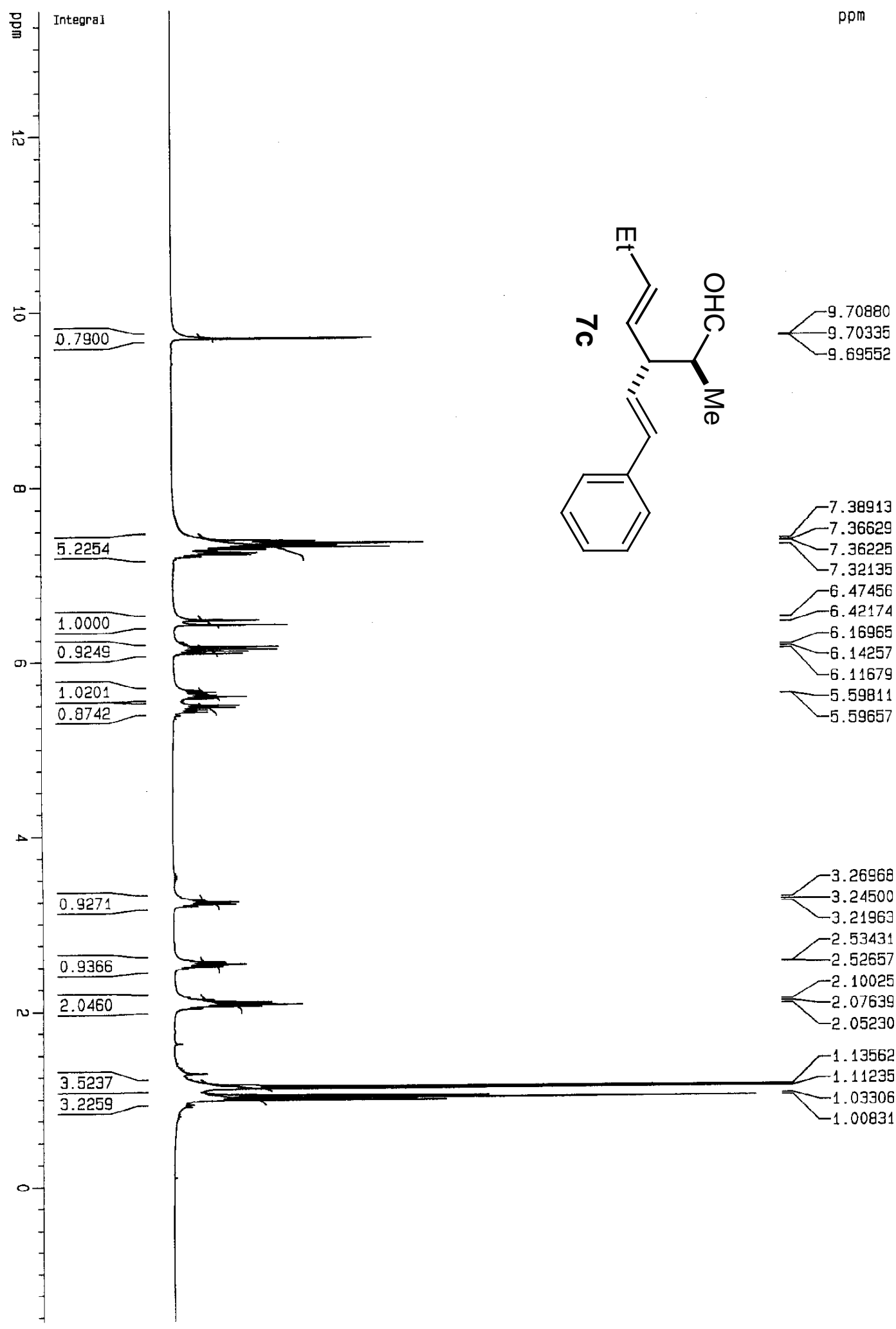

$\Sigma$
승
0
0
0
0
0
0
$\omega$
0
0 


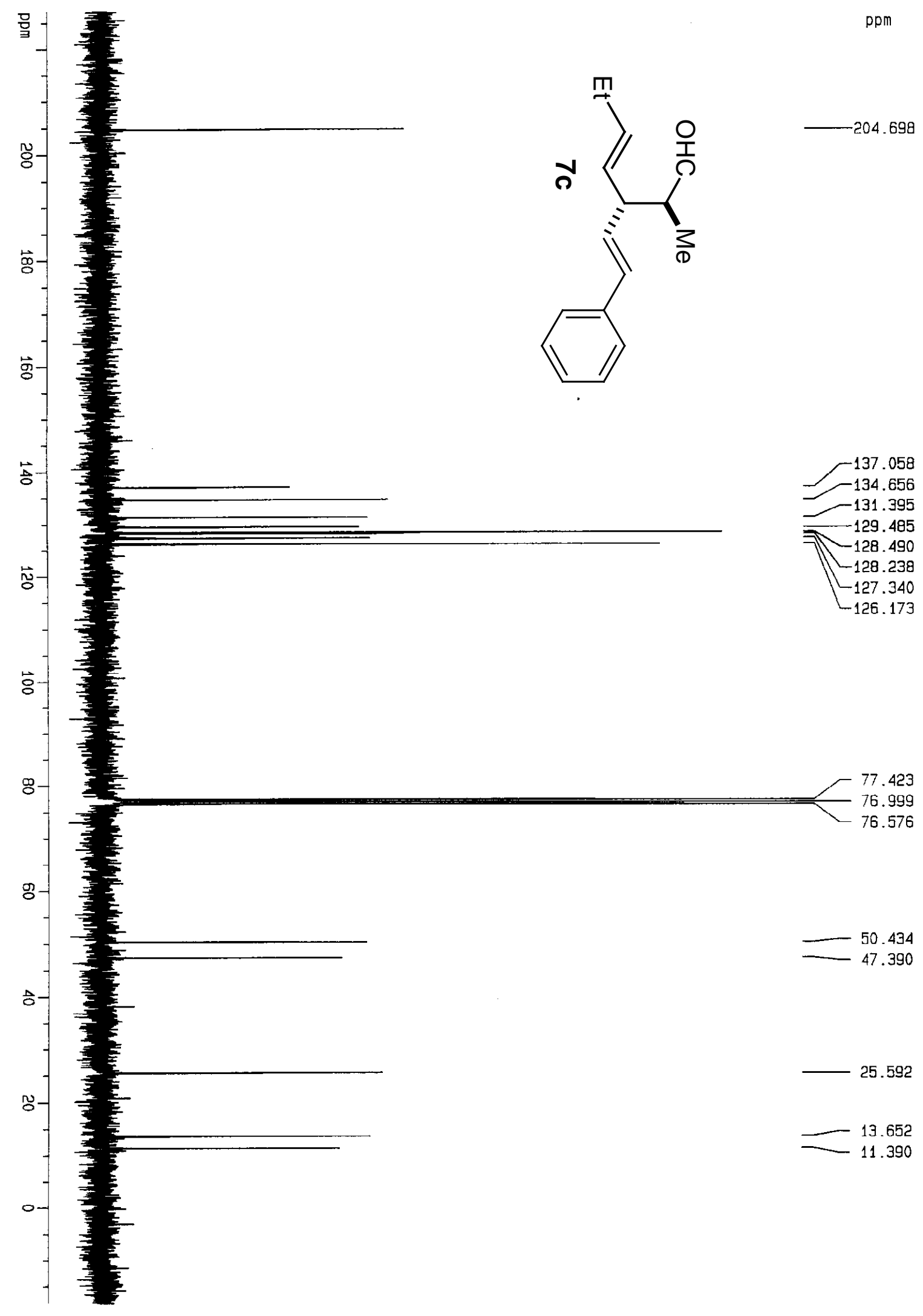



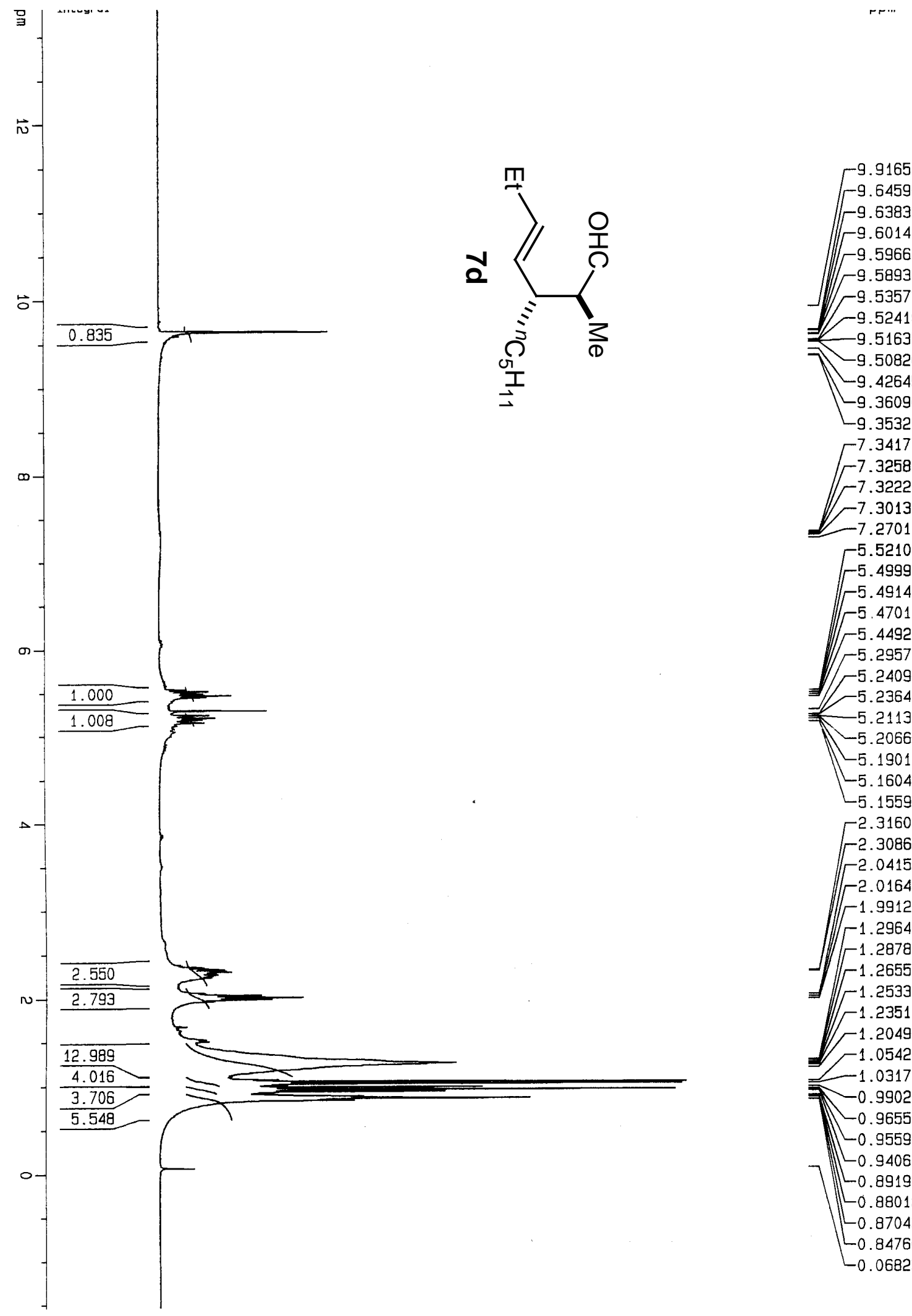

7.34177

$-7.32586$

$-7.32226$

$-7.30133$

$-7.27015$

5.52109

$-5.49998$

$-5.49142$

$-5.47016$

$-5.44920$

$-5.29571$

$-5.24096$

$-5.23642$

$-5.21131$

$-5.20662$

$-5.19019$

$-5.16048$

$-5.15595$

-2.31605

$-2.30863$

$-2.04156$

-2.01641

$-1.99127$

$-1.29648$

$-1.28784$

- 1.26550

$-1.25333$

$-1.23510$

$-1.20494$

$-1.05424$

$-1.03170$

$=0.99021$

$-0.96557$

$-0.95599$

$-0.94069$

$-0.89194$

$-0.88018$

$-0.87043$

$L_{0.84760}$

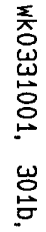

$L_{0.06823}$ 


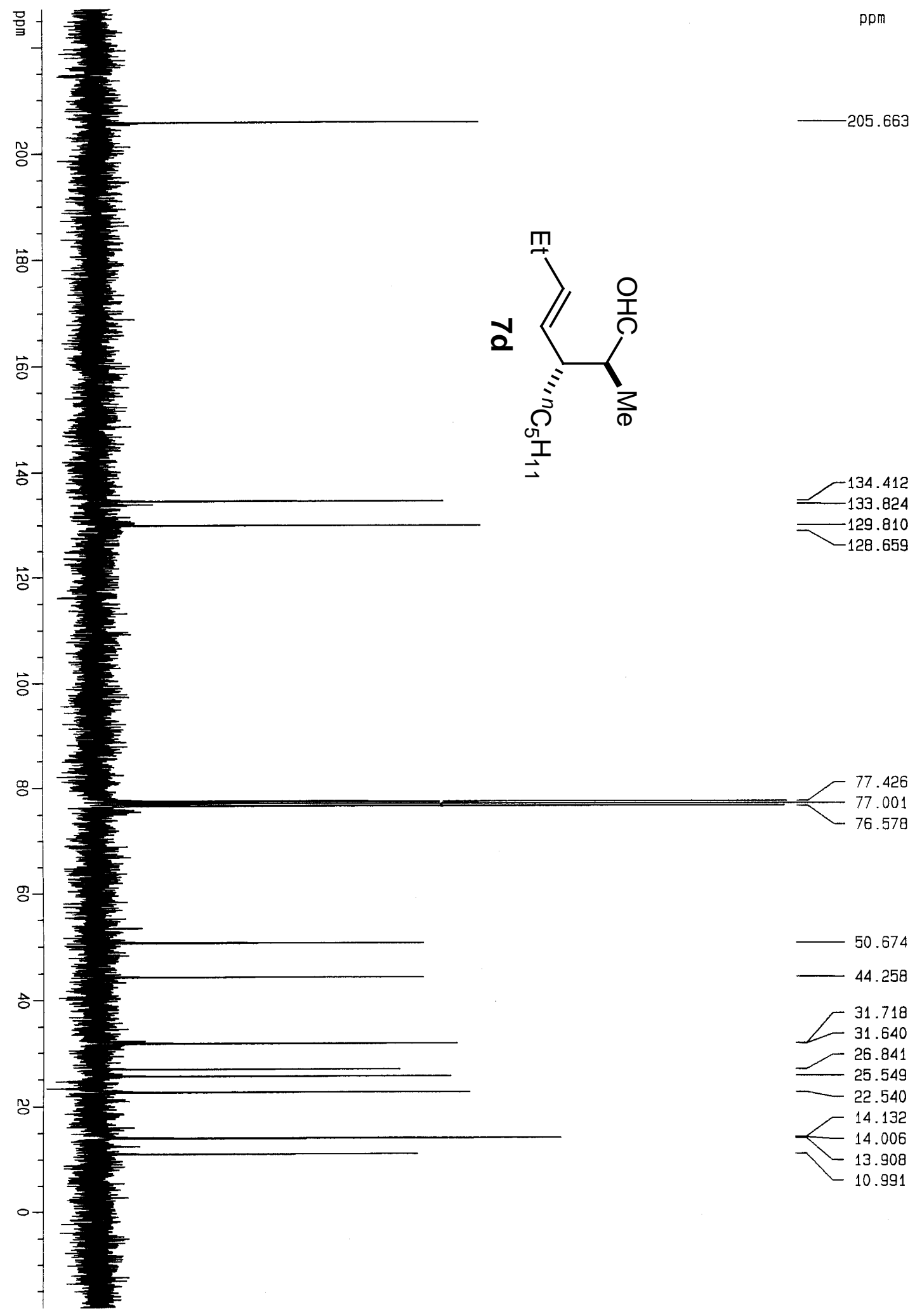




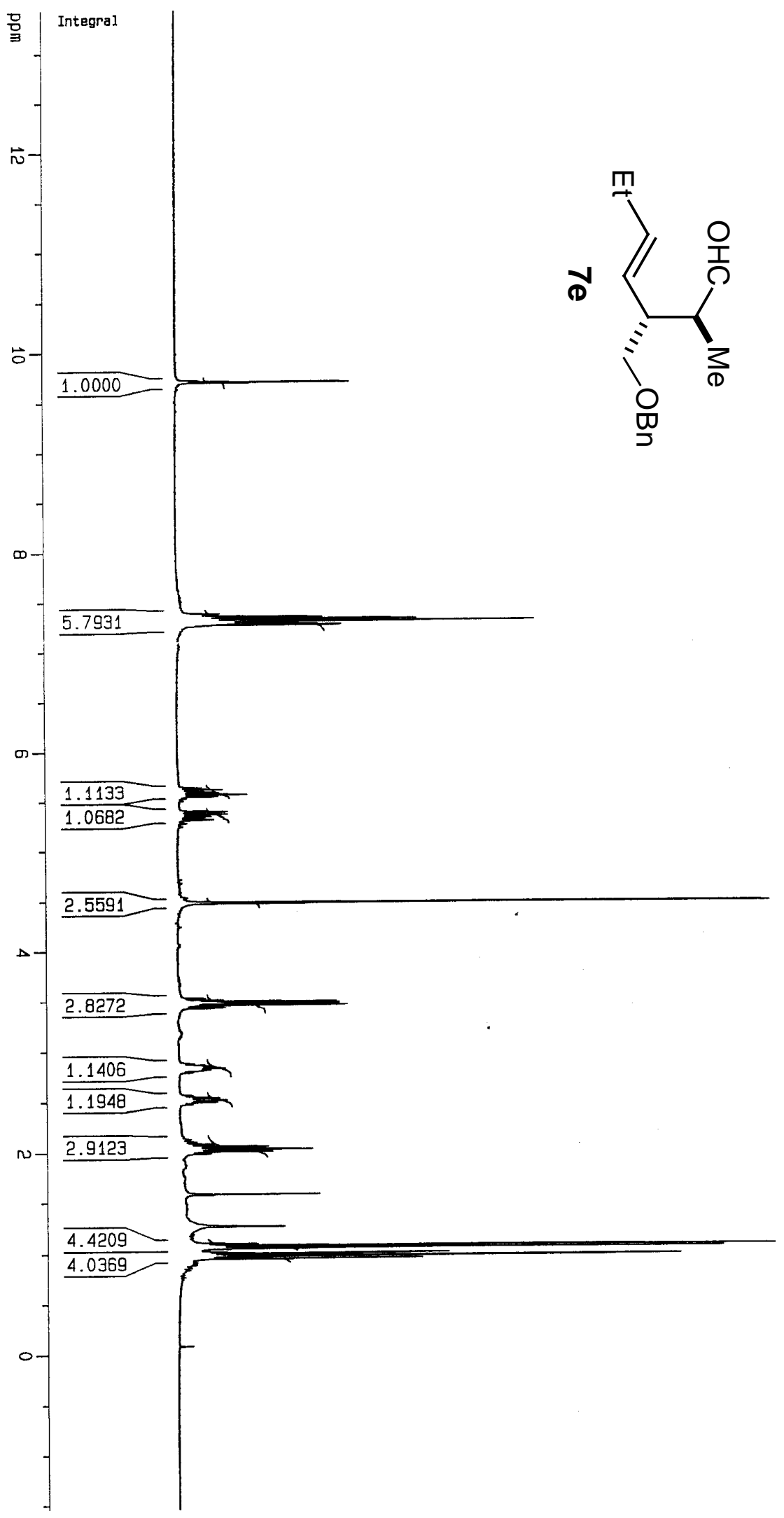

ppm

$\left[\begin{array}{r}9.80656 \\ -9.71836 \\ -9.71541 \\ -9.70602 \\ \Gamma^{-9.70462} \\ -9.70206 \\ -9.66722 \\ -9.63336 \\ -9.62567\end{array}\right.$

7.61462

$-7.37639$

$-7.34965$

$-7.34270$

$-7.32894$

$-7.30665$

$-7.28270$

$-7.26978$

$-7.26815$

$-5.61905$

5.58865

$-5.56762$

$-5.54686$

$-5.39415$

$-5.36662$

$-5.31520$

= 4.47954

$-3.50539$

$-3.49362$

$-3.48252$

$-3.47452$

-3.45622

$-3.43135$

$-3.42532$

-2.83551

$-2.54026$

$-2.51010$

$-2.51359$

$-2.05854$

$-2.03368$

2.00866

$-1.57937$

$-1.26330$

$-1.09373$

$-1.07184$

$7-1.04893$

$-1.00954$

$-1.00170$

$7 \quad-0.98453$

L 0.97694

- 0.96028

Lo.95208

Lo.83486

$-0.07969$ 


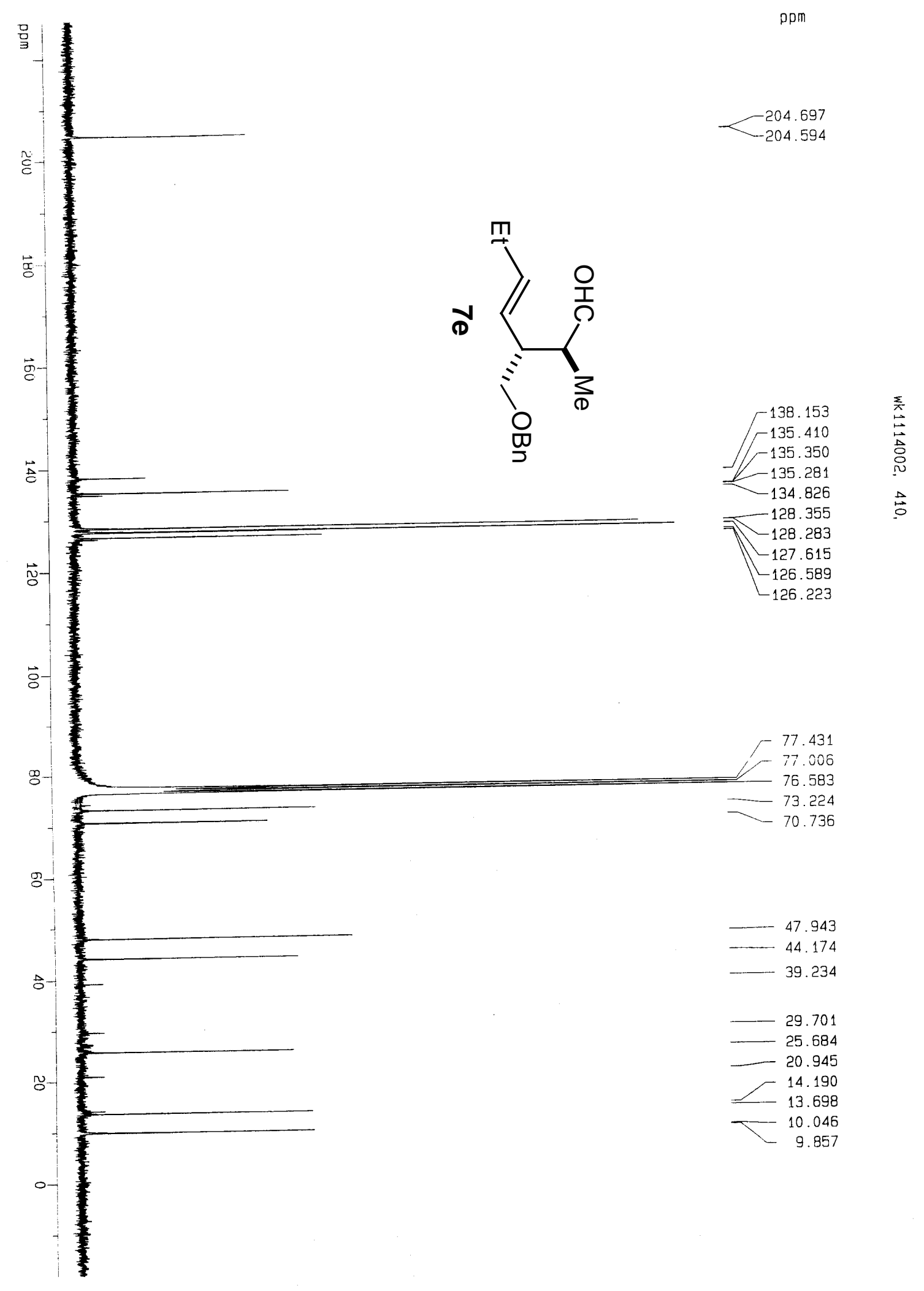




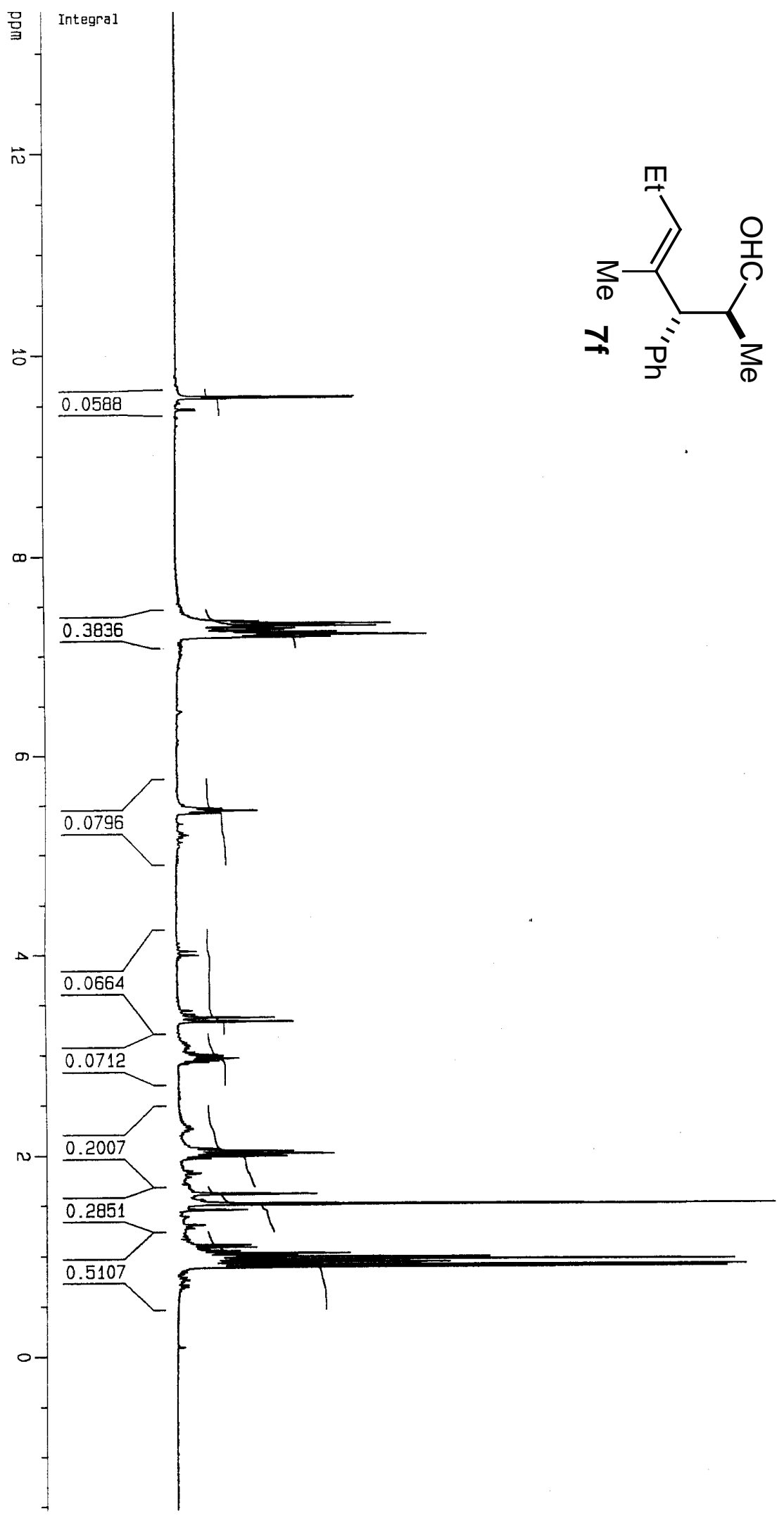

$-9.45216$

$-7.55780$

$-7.34434$

$-7.33757$

$-7.32135$

-7.29570

$-7.27171$

$-7.27010$

$-7.26592$

$-7.26064$

$-7.25598$

$-7.23660$

$-7.22555$

$-7.21496$

$-7.21037$

-7.20197

$L_{7.18722}$

$=5.46186$

$-5.43885$

$-5.41569$

-3.36810

-3.33021

$-2.98541$

$-2.97599$

$-2.96158$

$=-2.93851$

$-2.04006$

$-2.01590$

$-1.99111$

$-1.60965$

$r^{1.50688}$

$-1.45137$

$-1.10559$

$-1.08289$

$-1.04532$

1.02092

$-1.01513$

$-0.99958$

-0.98524

$7-0.96023$

0.93507

- 0.90911

L 0.88634

$L_{0.75979}$

$L_{0.10559}$

$L_{0.08657}$ 

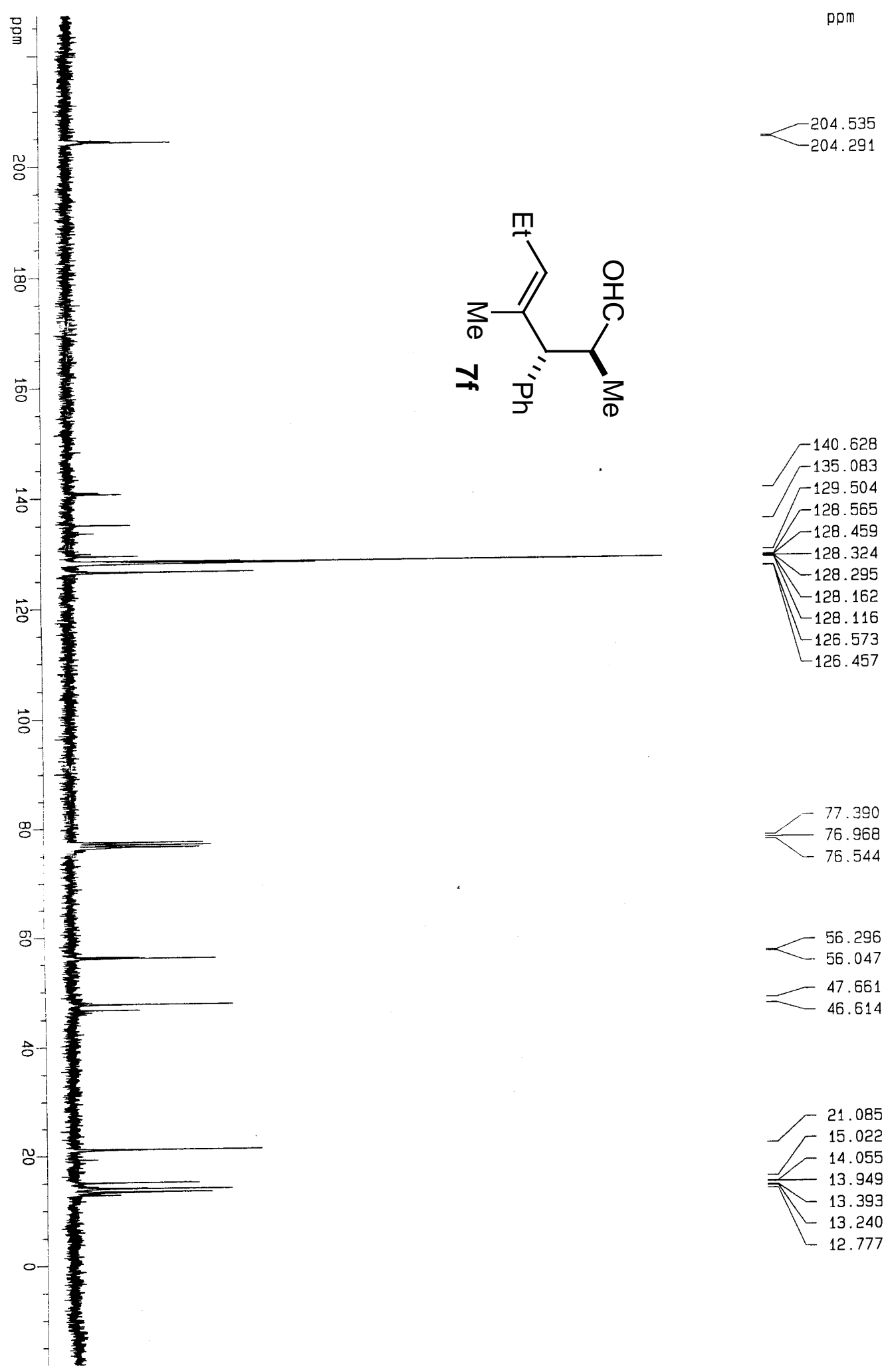

77.390

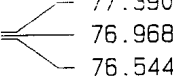

$-56.296$

$-56.047$

$-47.661$

$-46.614$

21.085

$\Gamma^{15.022}$

14.055

13.949

$-13.393$

$-13.240$

12.777 


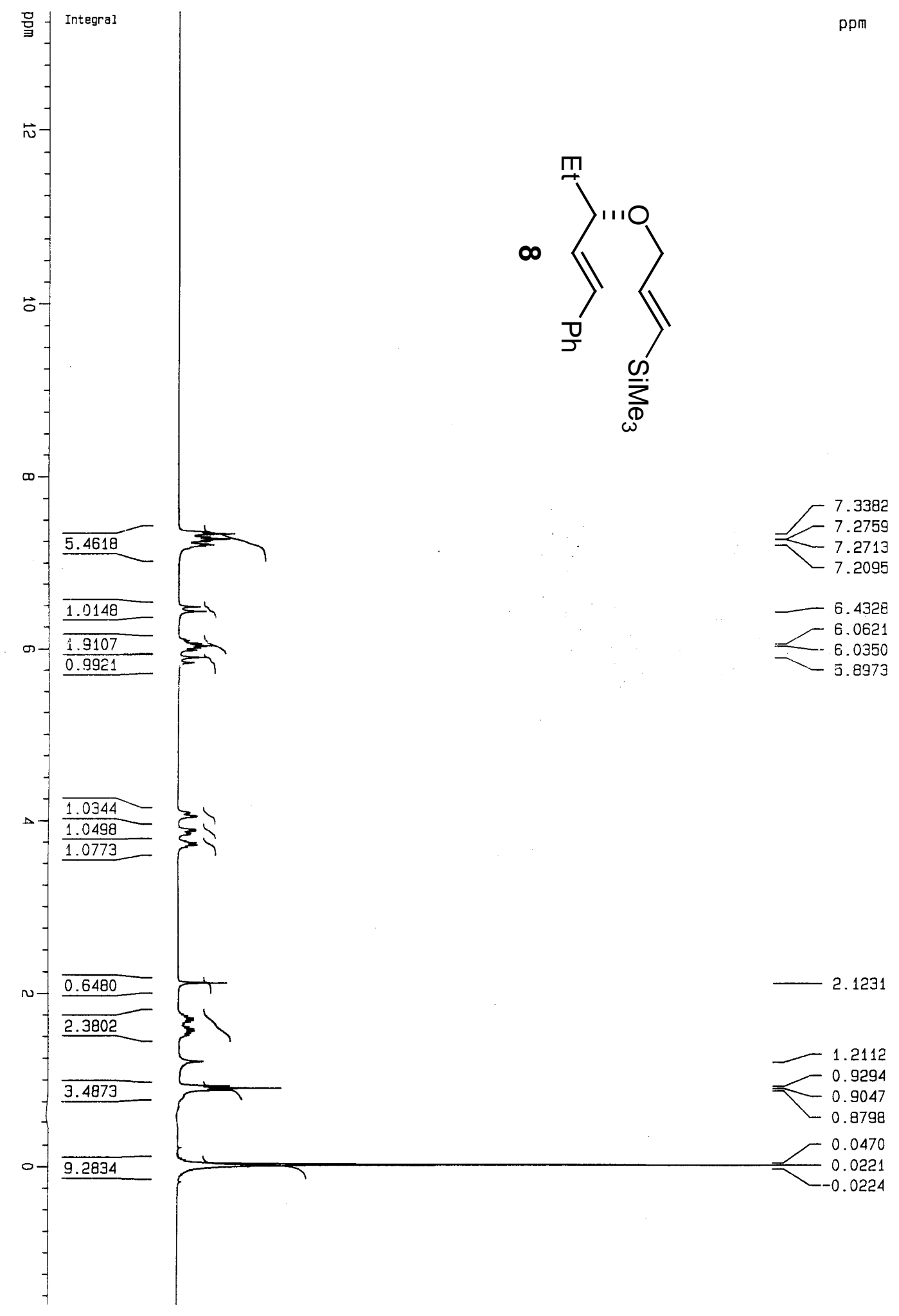




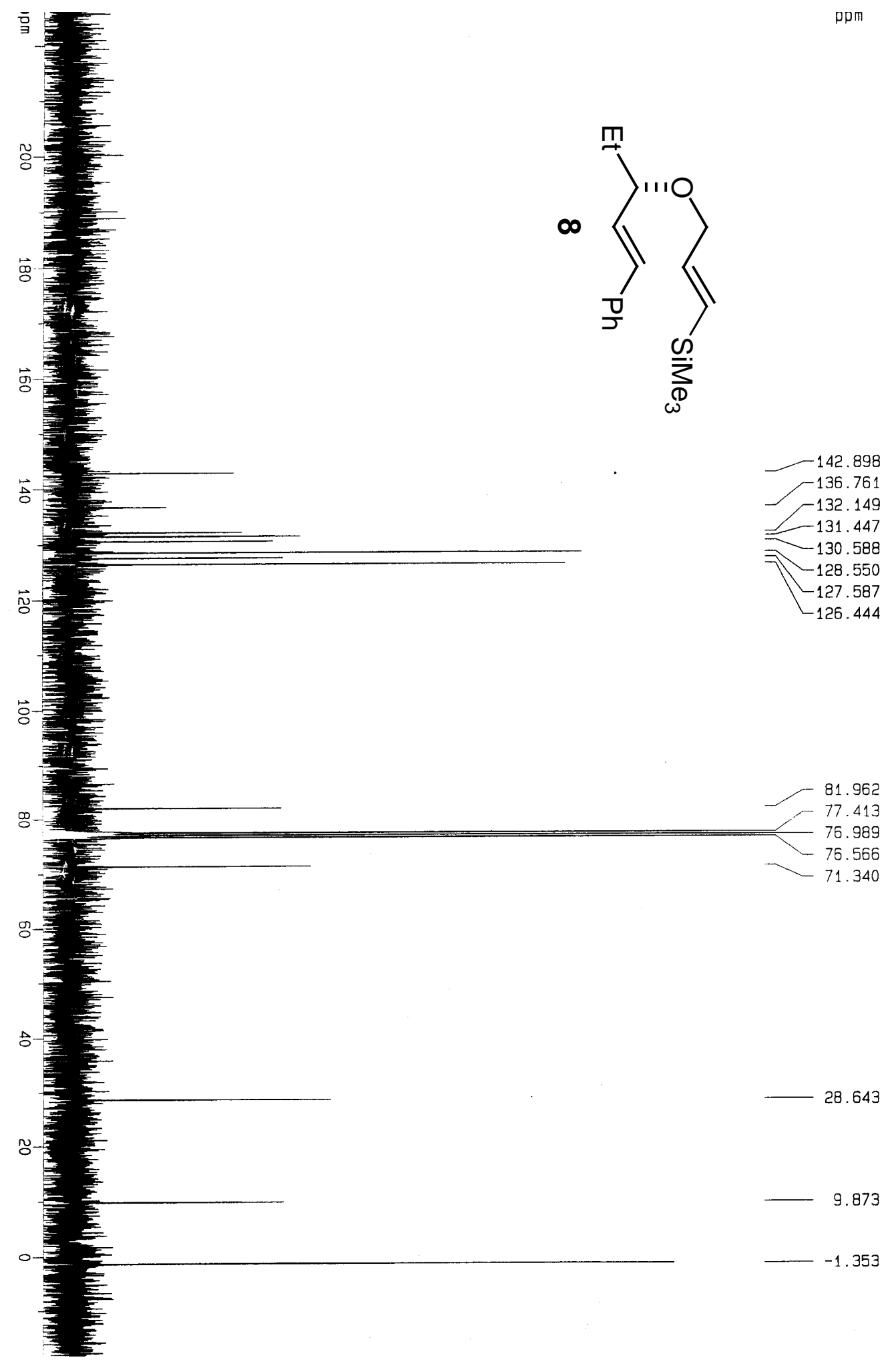




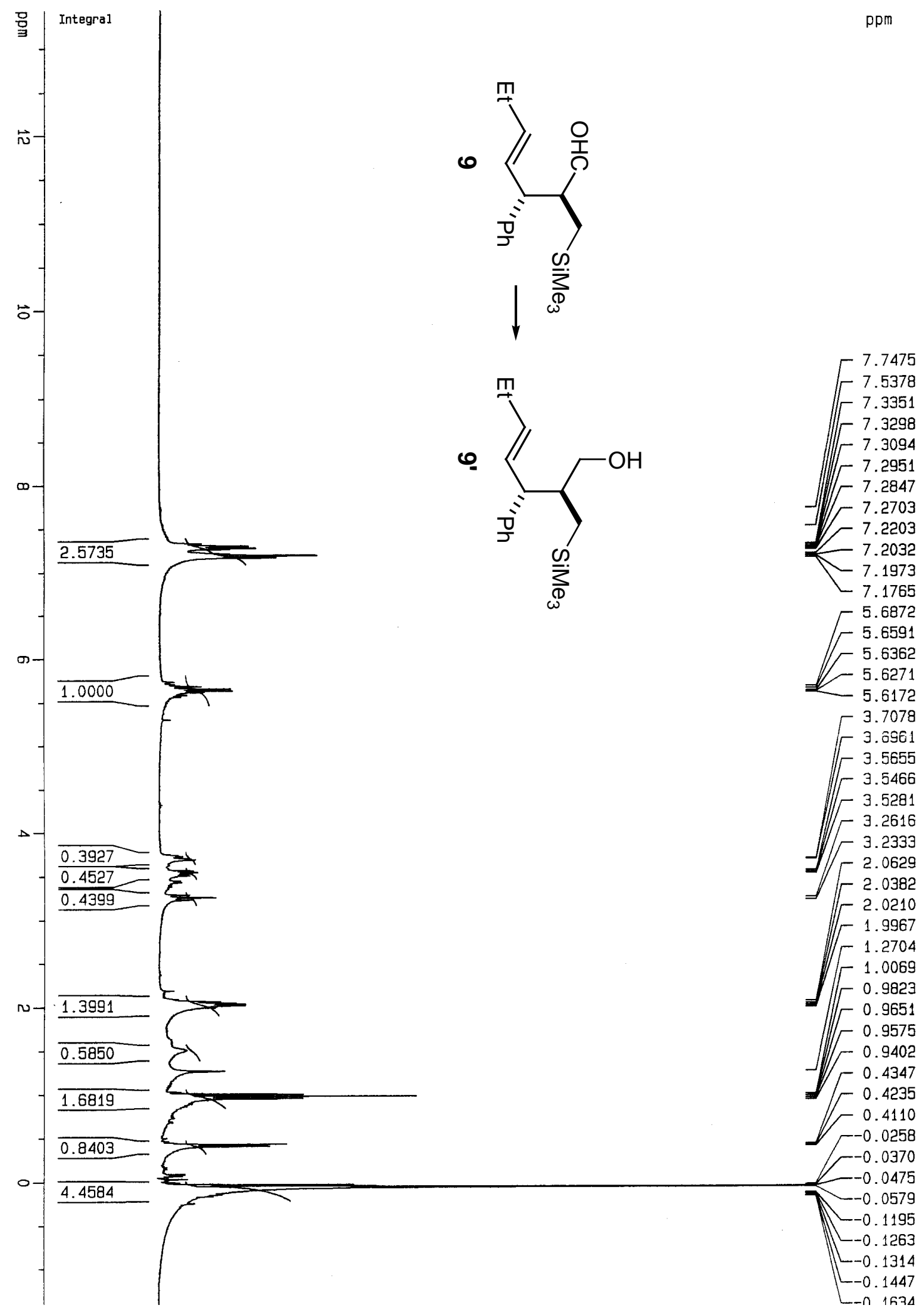



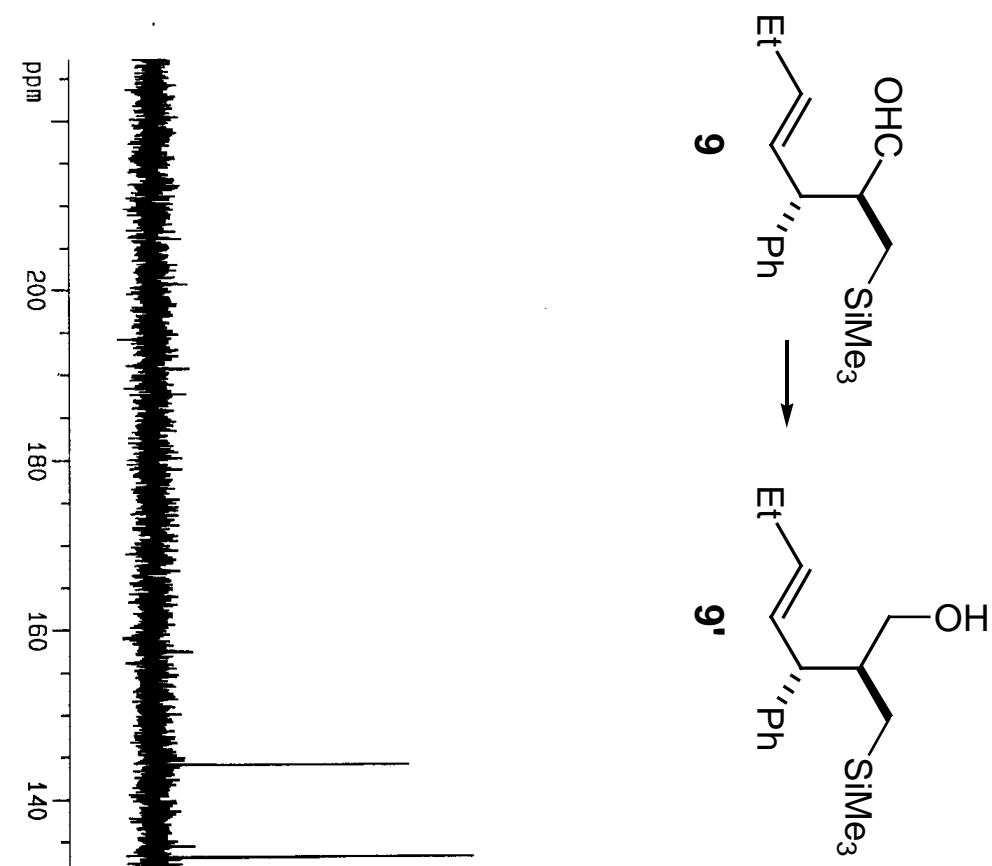


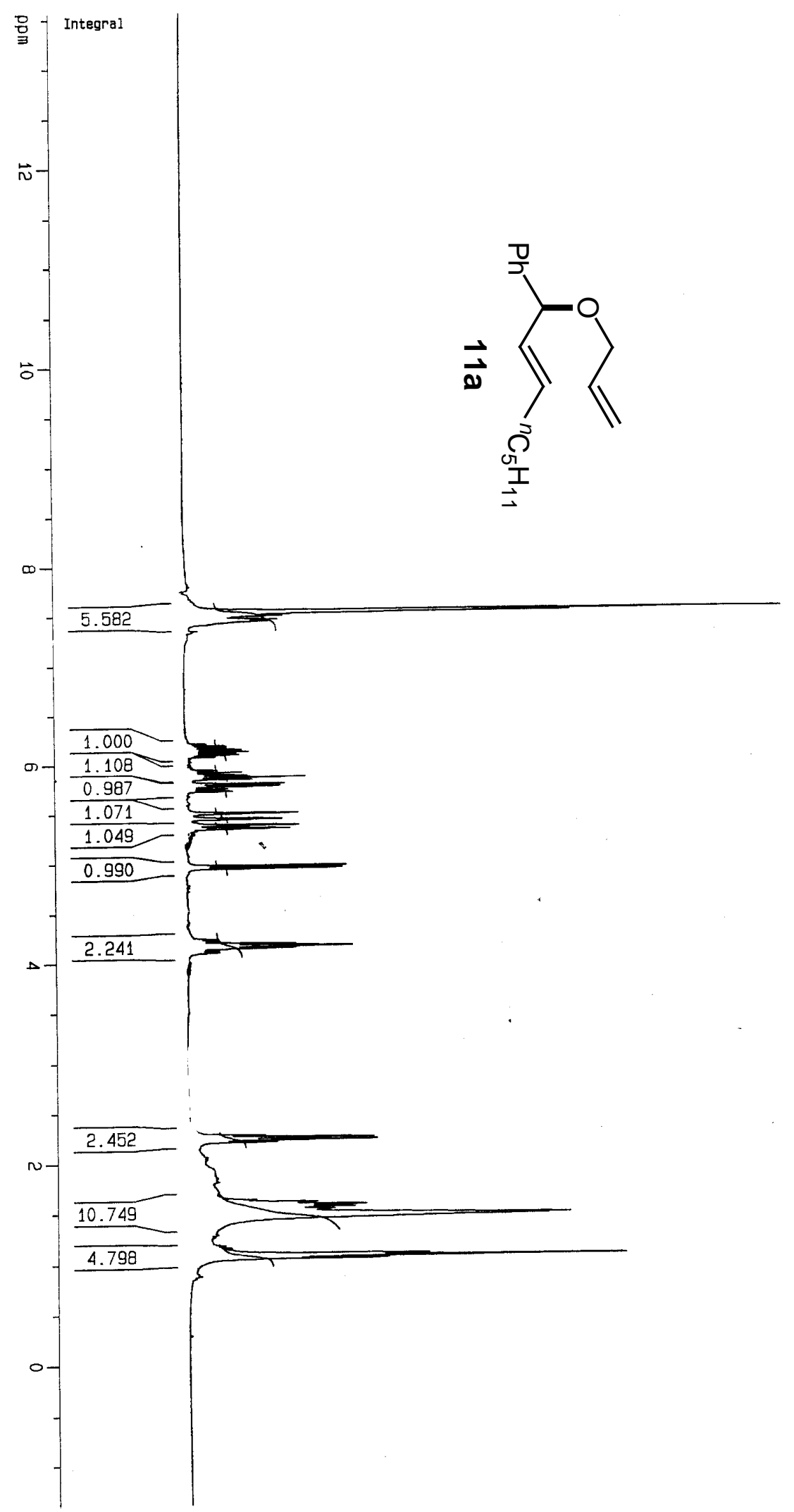

$-11.8224$

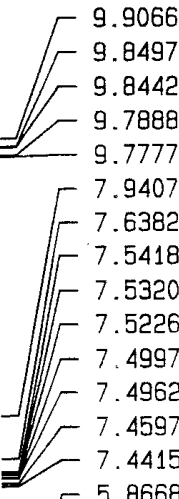

- 5.8668

5.7986

$-5.7956$

$-5.7750$

5.7719

5.5006

$J-5.4979$

$-5.4432$

5.3802

$-5.3785$

$-5.3456$

$-5.3440$

$-4.9714$

$-4.9479$

$-4.1905$

$-4.1867$

$-4.1676$

$-4.1652$

$-4.1464$

L 4.1435

$-2.2525$

$-2.2285$

$-2.2054$

1.6059

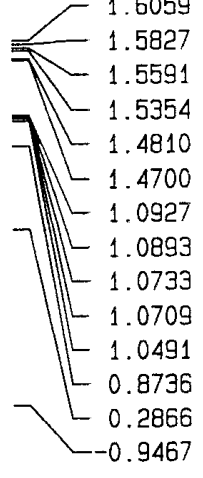



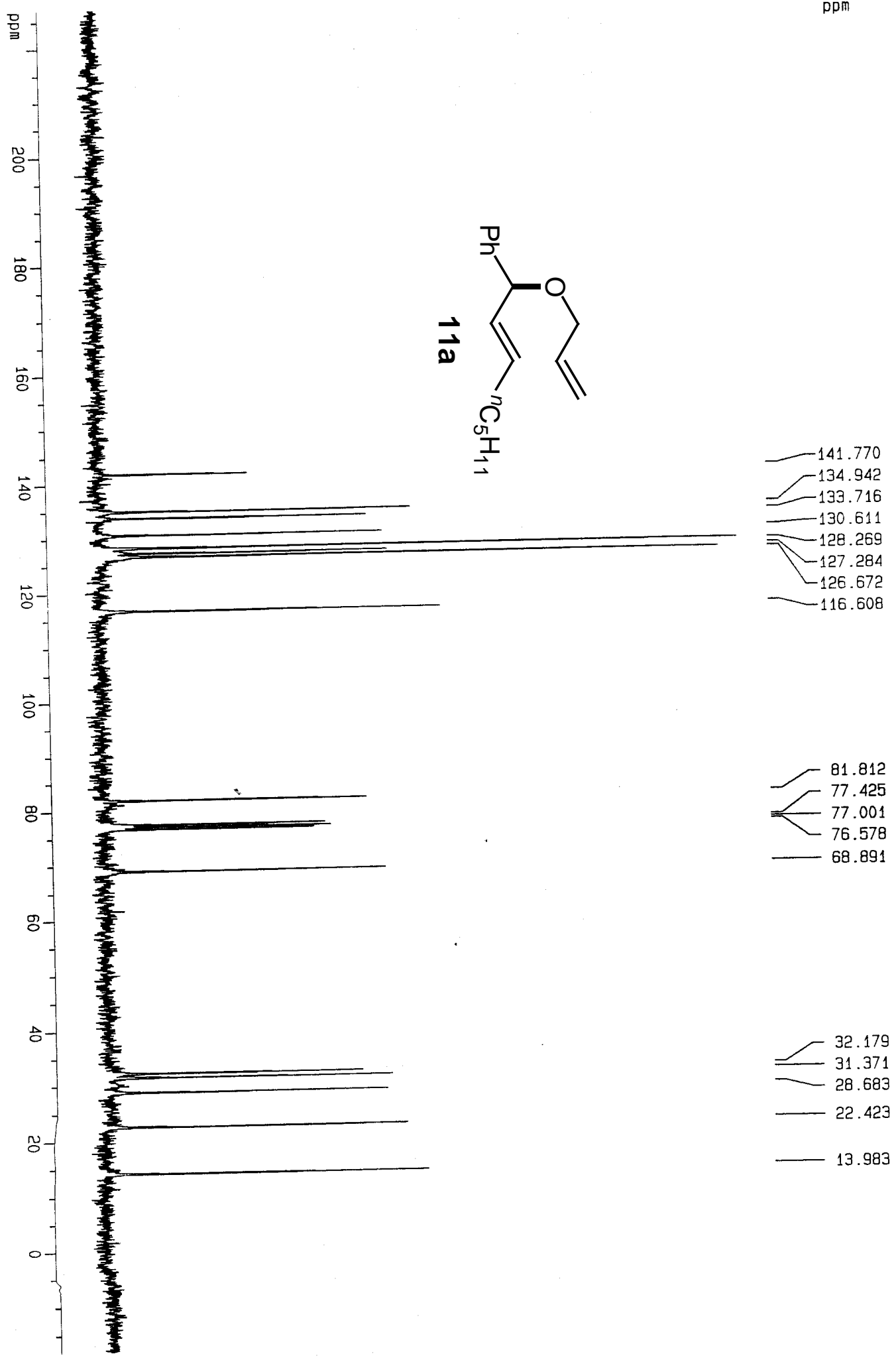

81.812

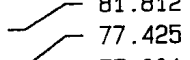

$-77.001$

76.578

68.891

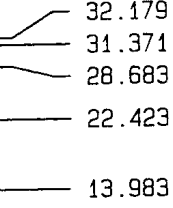




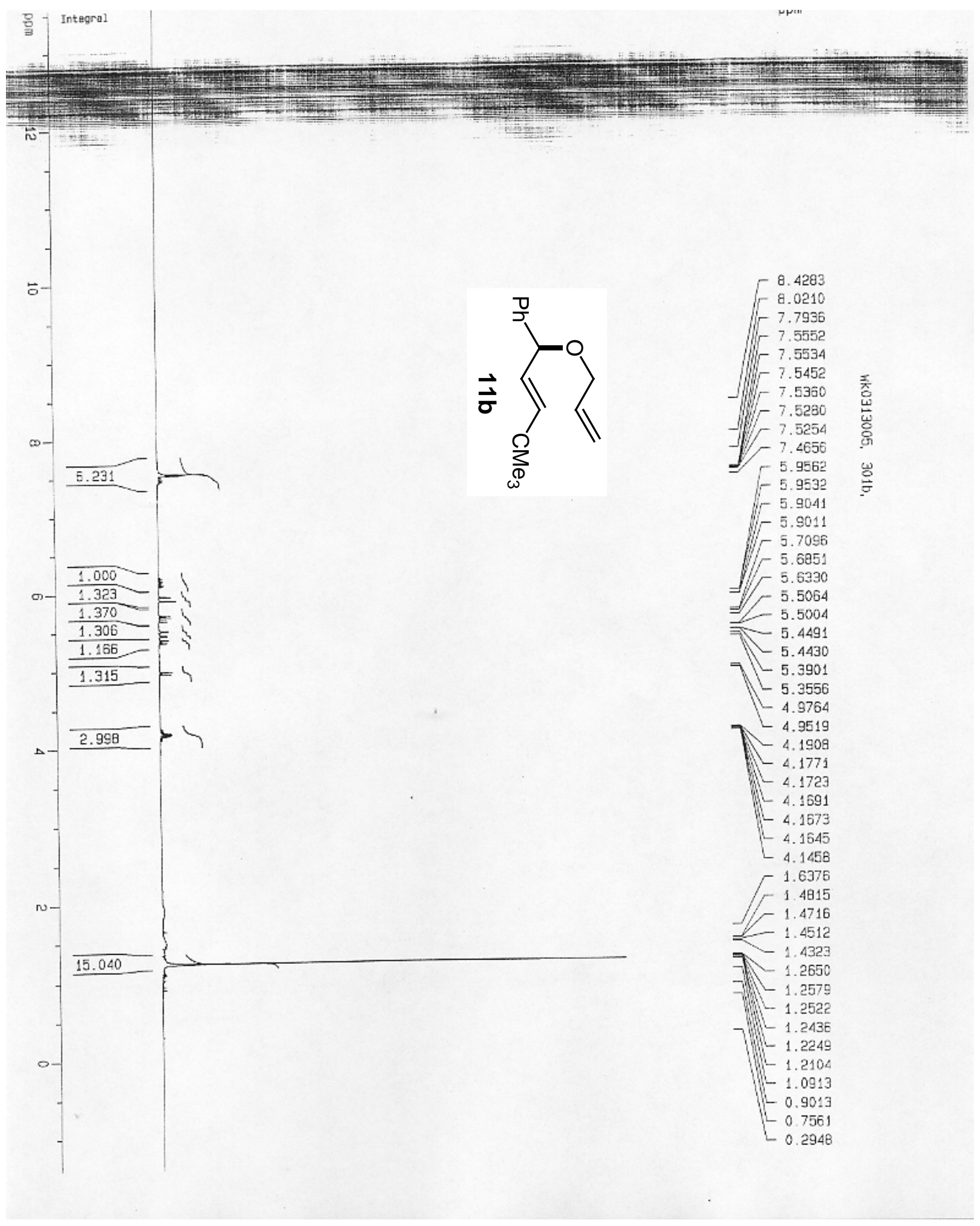




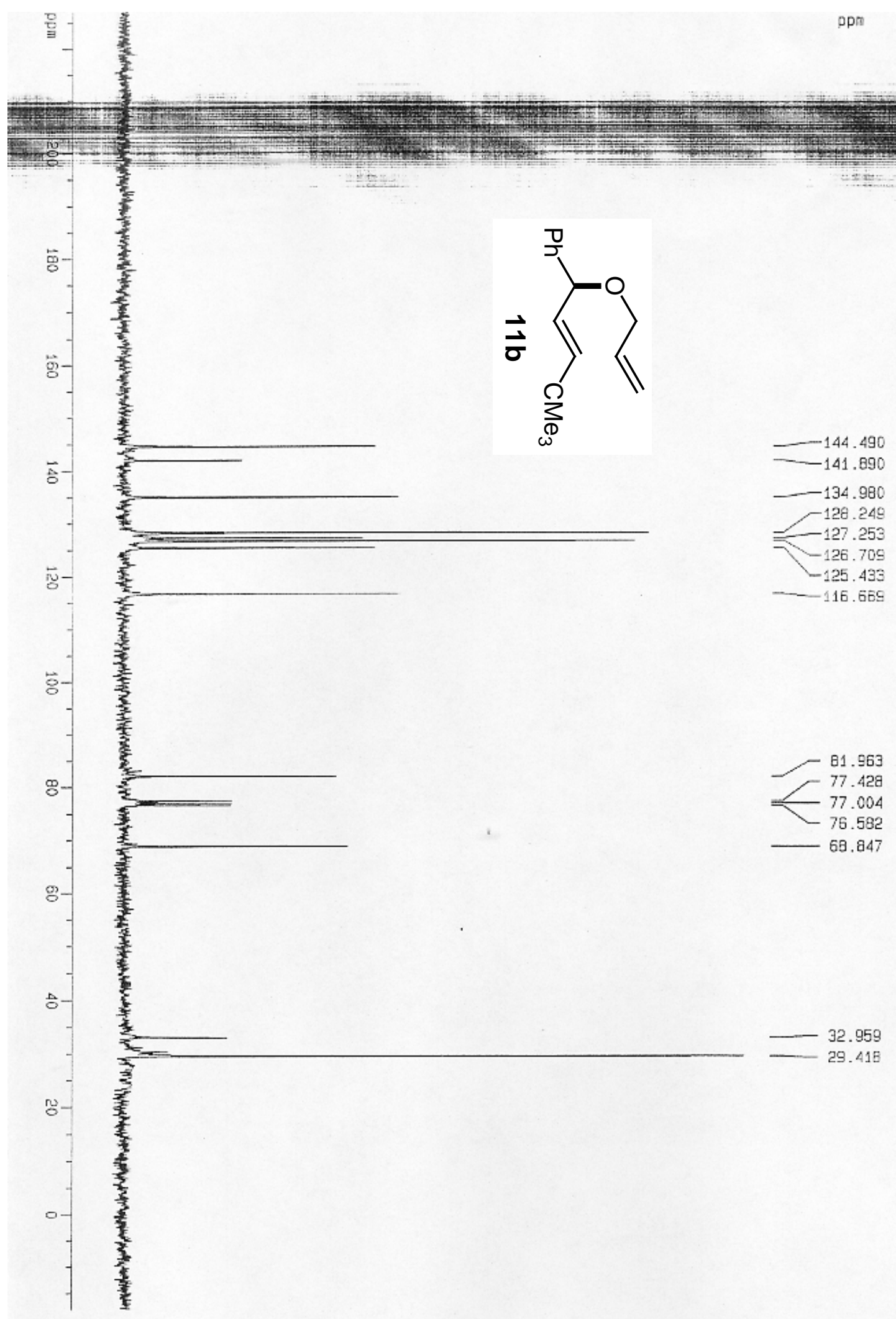




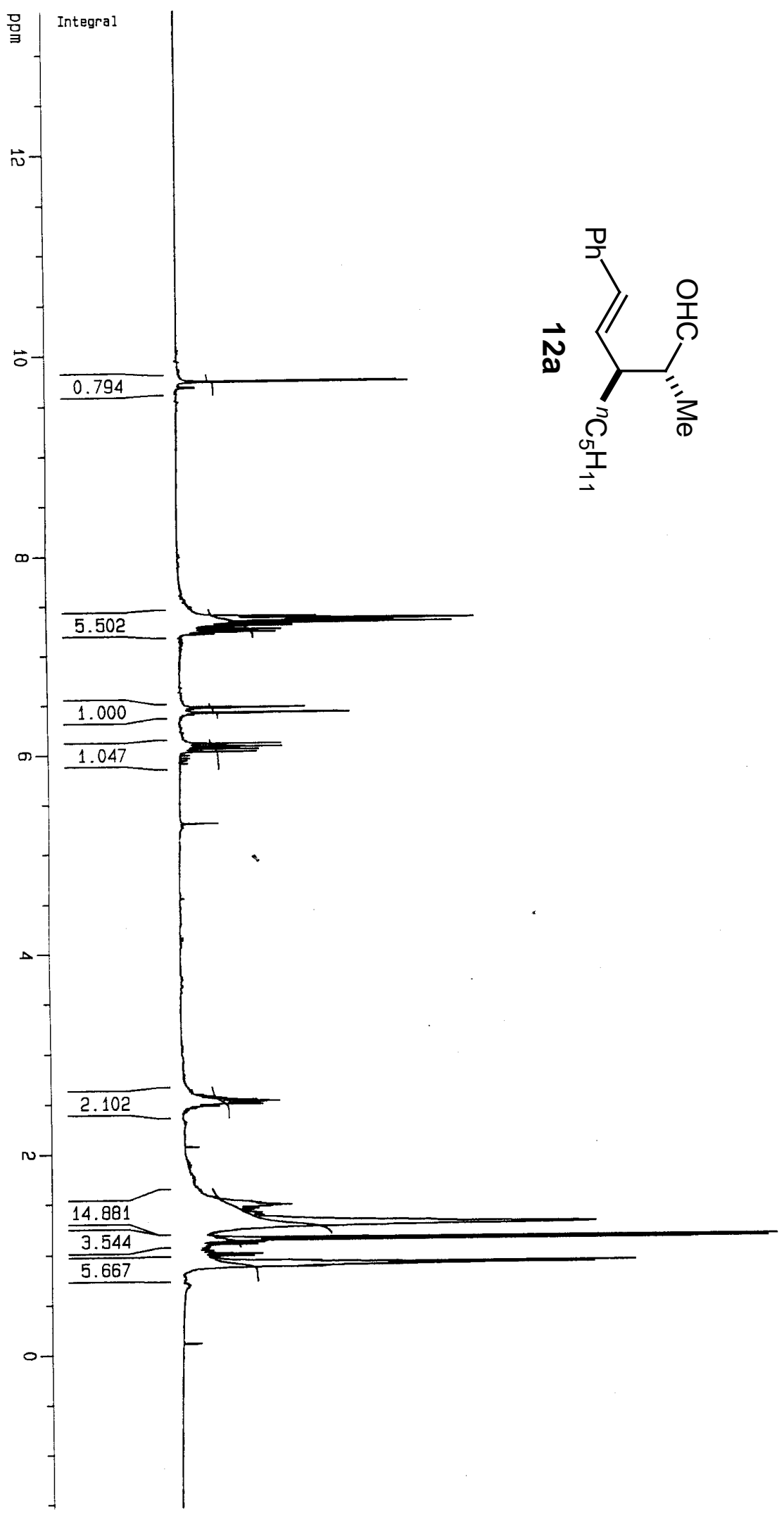

ppm

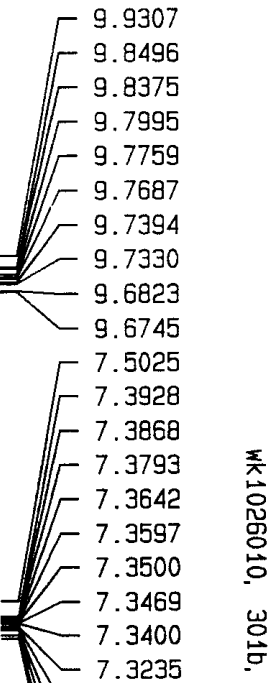

๑ 7.3033

$-7.2973$

皮 $\quad 7.2589$

L 7.2351

L 6.4716

L 6.4189

$-6.1088$

6.0789

$\leftarrow 6.0562$

$-6.0263$

$-2.5229$

$-2.5162$

- 2.5006

- 2.4940

$\int\left[\begin{array}{l}1.4824 \\ 1.3203 \\ 1.3167\end{array}\right.$

] 1.2974

I 1.2935

- 1.2840

$-1.2748$

1.2535

$-1.1535$

$-1.1310$

$-0.9308$

$-0.9262$

$-0.9157$

]$[-0.9092$

$\leftarrow 0.8938$

$-0.8863$

$-0.8717$

$[0.6843$

$\leftarrow 0.1149$

$-0.1025$ 


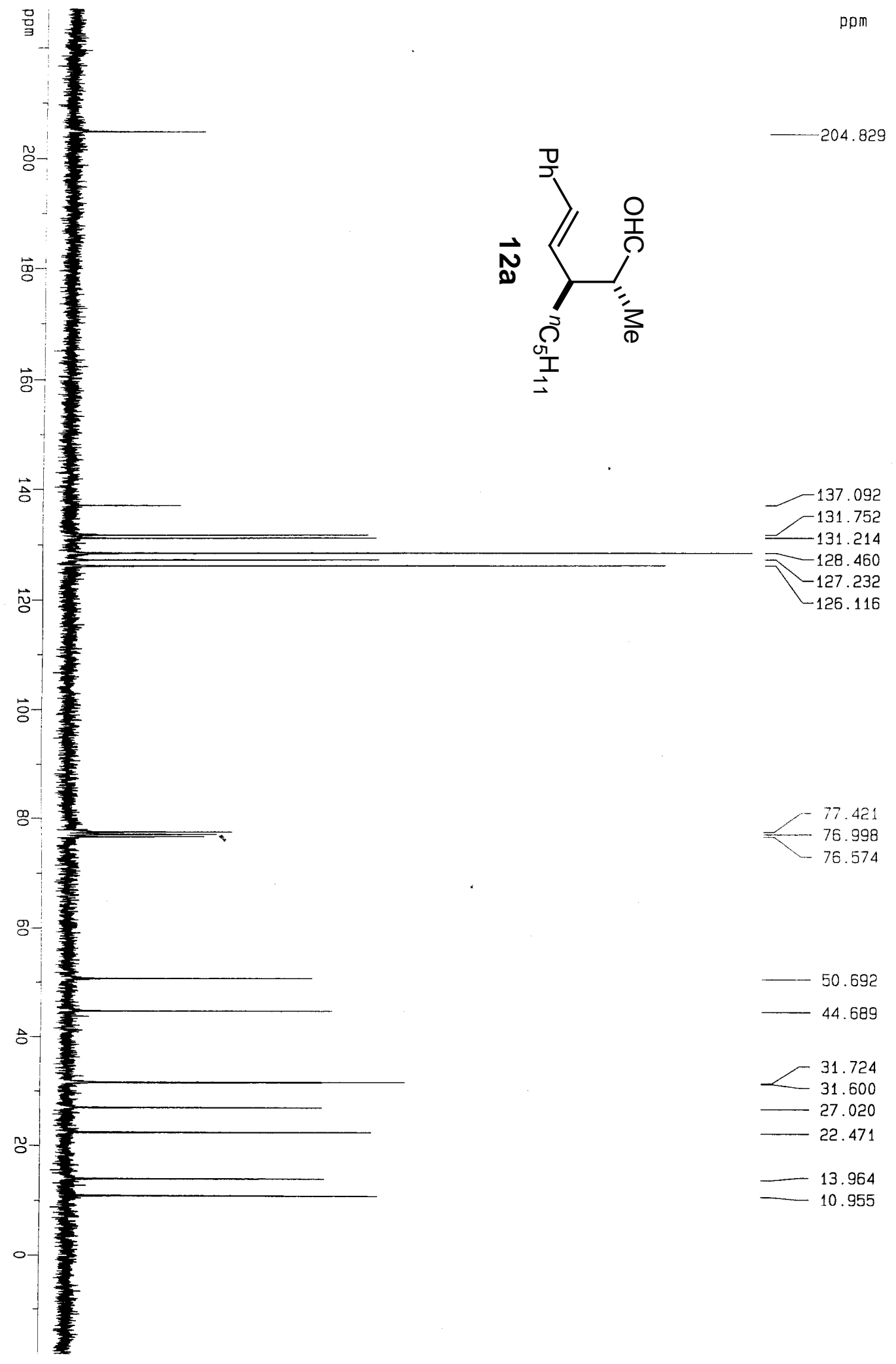



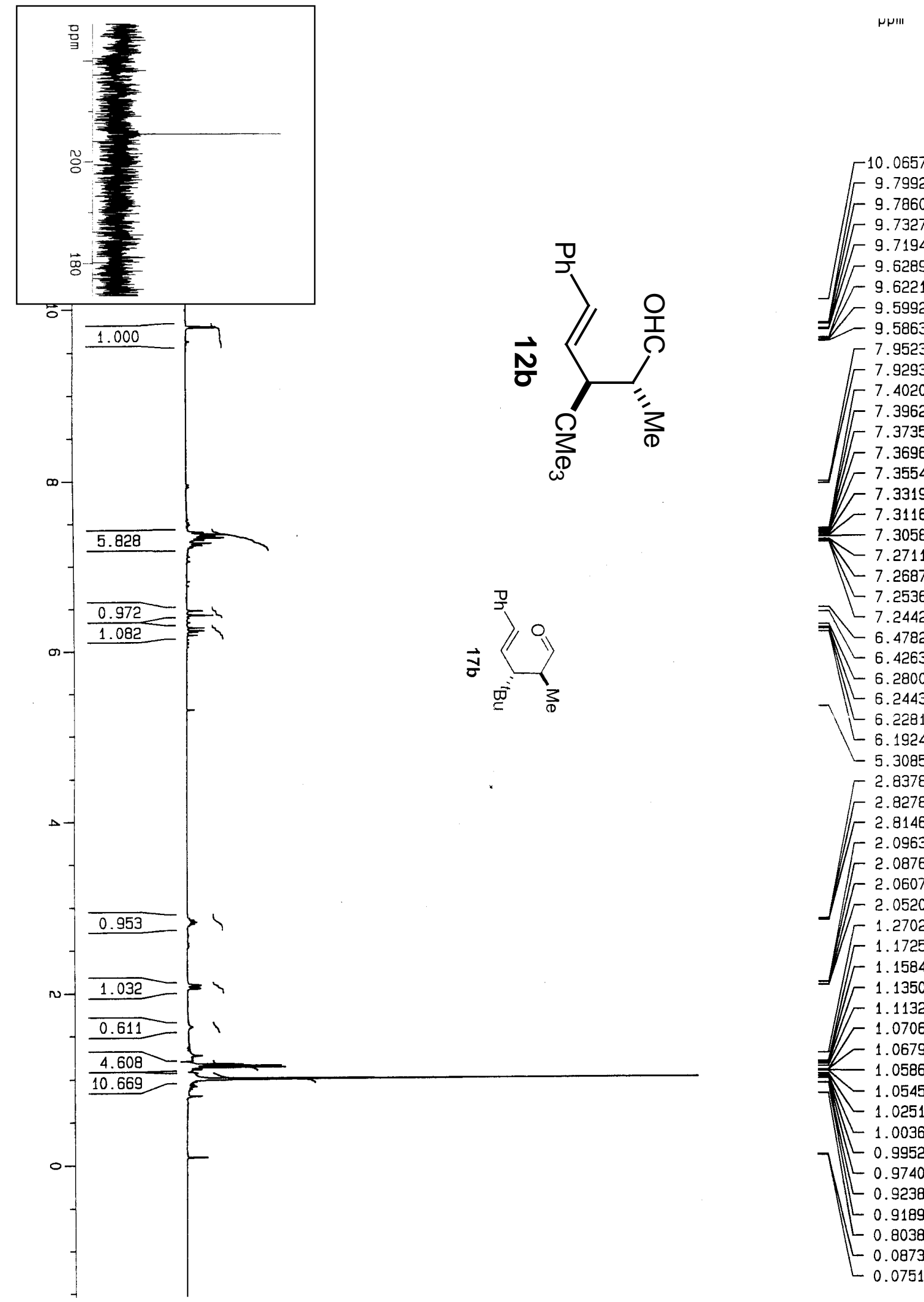

- 7.9523

- 7.9293

7.4020

7.3962

- 7.3735

- 7.3696

] -7.3554

7.3319

$-7.3116$

7.3058

$-7.2711$

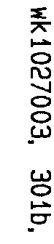

$-7.2536$

ר $\lfloor 7.2442$

$7-6.4782$

$-6.4263$

$-6.2800$

$-6.2443$

- 6.2281

L 6.1924

$-5.3085$

2.8378

$-2.8278$

- 2.8146

2. 0963

$\int\left[\begin{array}{r}2.0876 \\ 2.0607\end{array}\right.$

] $\begin{array}{r}2.0520 \\ 1.2702\end{array}$

$-1.2702$

- 1.1725

] 1.1584

F 1.1350

F 1.1132

F 1.0706

$-1.0679$

$-1.0586$

$7 \longleftarrow \begin{aligned} & 1.0545 \\ & 1.0251\end{aligned}$

- 1.0036

L 0.9952

L 0.9740

0.9238

L 0.9189

L 0.8038

0.0873

L 0.0751 


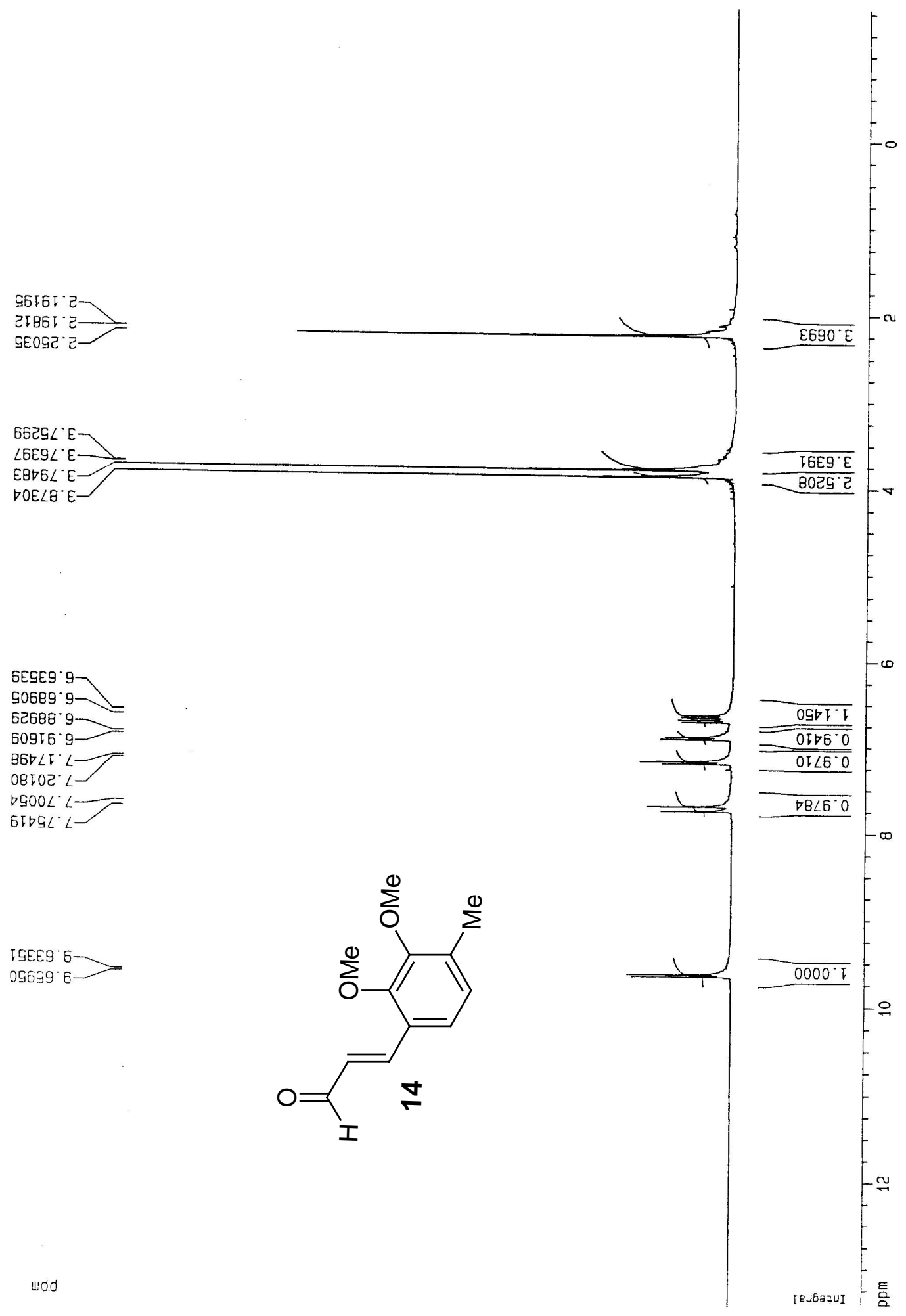


$200^{\circ} 91-$

G28.69
EB8.09

$G \angle 9^{\circ} 9 \angle$ $000^{\circ} L L \longrightarrow$
$\mathrm{GLD}^{\circ} \mathrm{LL}$

BOE. टट丁-

G66. $92 \mathrm{~T}-$

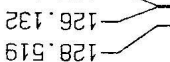

5IS'BटI-

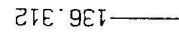

$\angle 9 E^{\circ} \angle \nabla I-$

EVG IST-
GLT.2GT

wdd

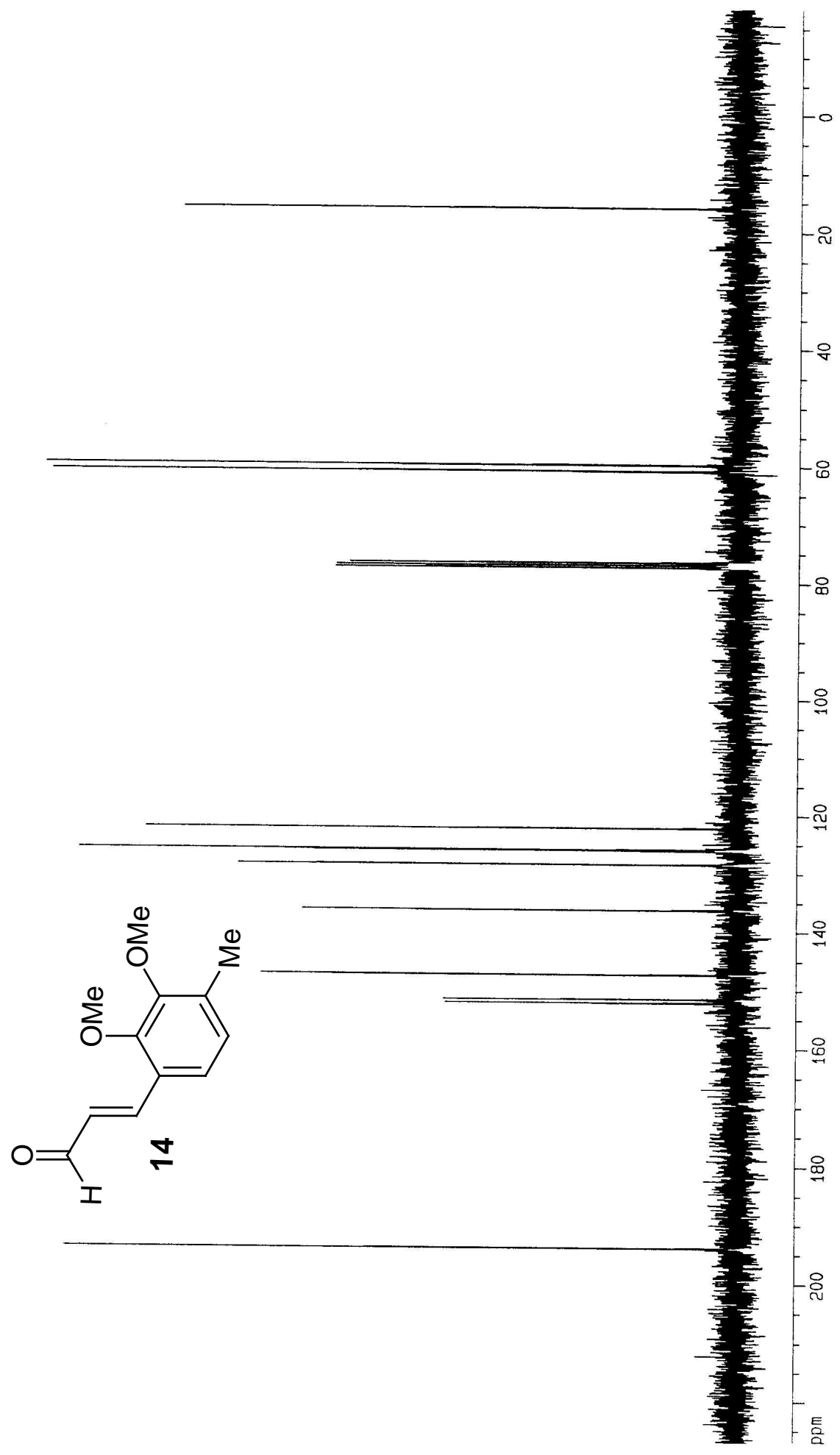




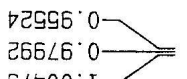

$9 \angle 000^{\circ}-7$

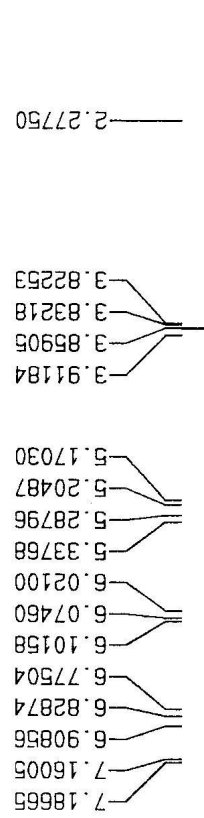

G009I $\angle \square=$
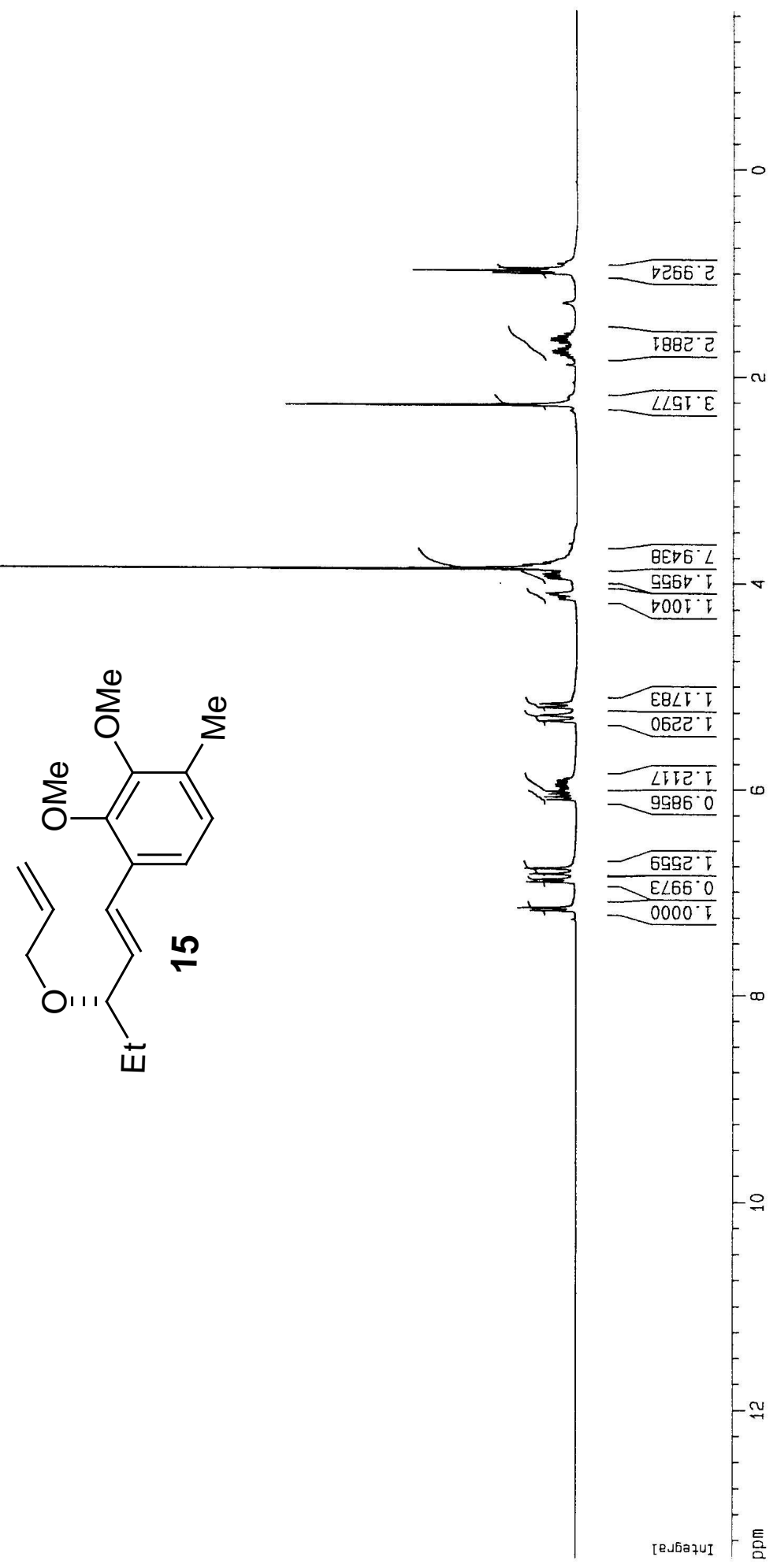
$918.6-$
$82 \angle .91-$
$629 \cdot 82-$

800.09 819.09

900.69 $\angle \angle G^{\circ} 9 \angle$

$100^{\circ} \mathrm{LL}$

$925 \cdot L L$

$\triangle D 5^{\circ} \mathrm{IB}$

86E. 91 I

926.0टt

BEL GLT

$E \nabla 9^{\circ} 951 \longrightarrow$

EI6.8टा-

$\angle B \angle O E I-T$

TEL GET-

OSG
OST
BDS IST

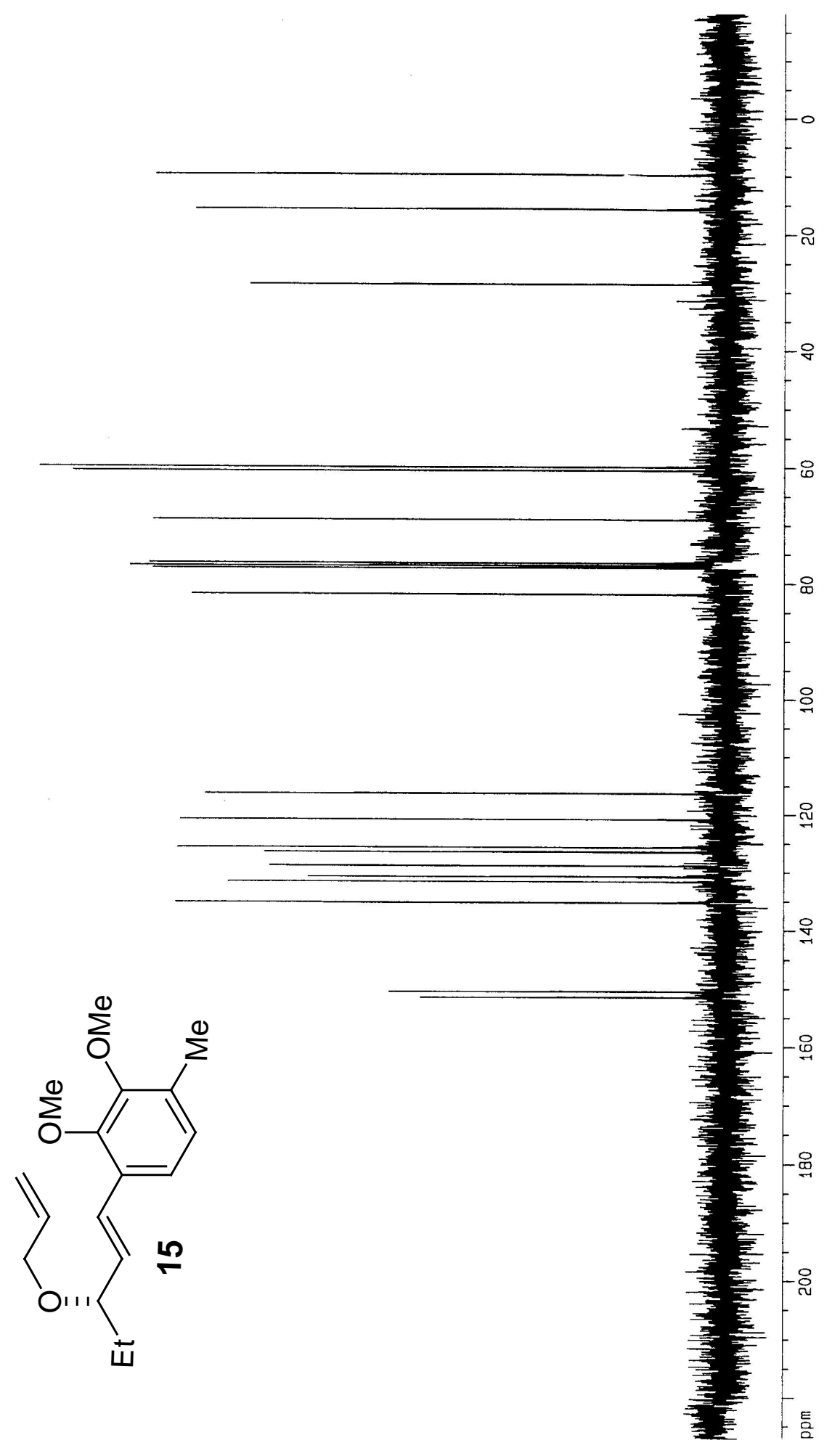




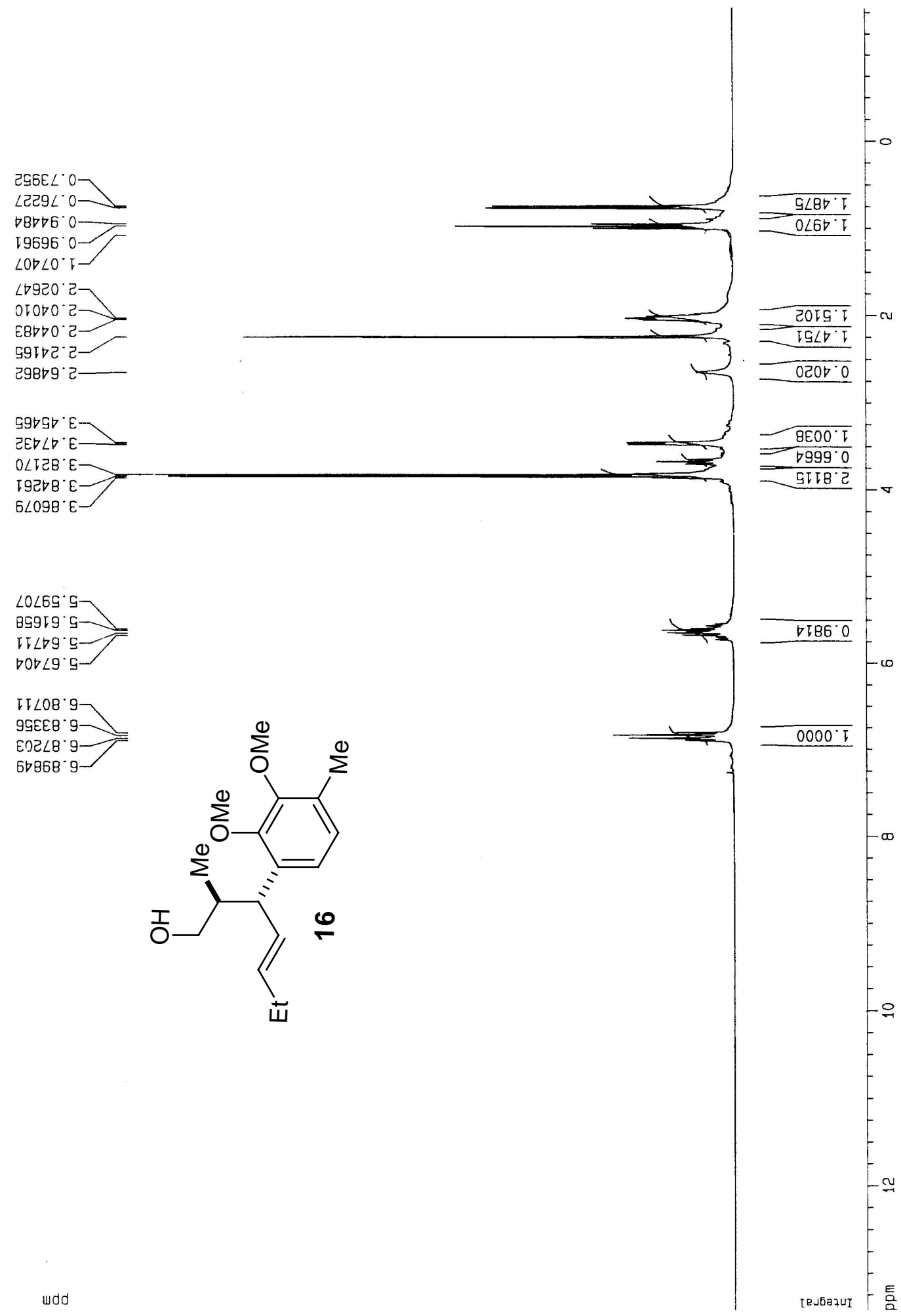


969. $E I$

$\nabla 68^{\circ} E[\longrightarrow$

$\mathrm{EOG}^{\circ} \mathrm{GI}$

$\angle G \nabla^{\circ} g 己$

IBC OD

$ट \angle D^{\circ} E \nabla+$

618.69

ELG.09

$999^{\circ} 99$-.

$\angle \angle G \cdot 9 \angle$

$200^{\circ} \angle L$

$920 .<1$

6टटनEटा—

$\angle 69^{\circ} \mathrm{GLI} \longrightarrow$

GI $\angle$ '62T-

EBE IEI TER

20t. $\triangle E I-$

869.05I

$691^{\circ}$ ISI

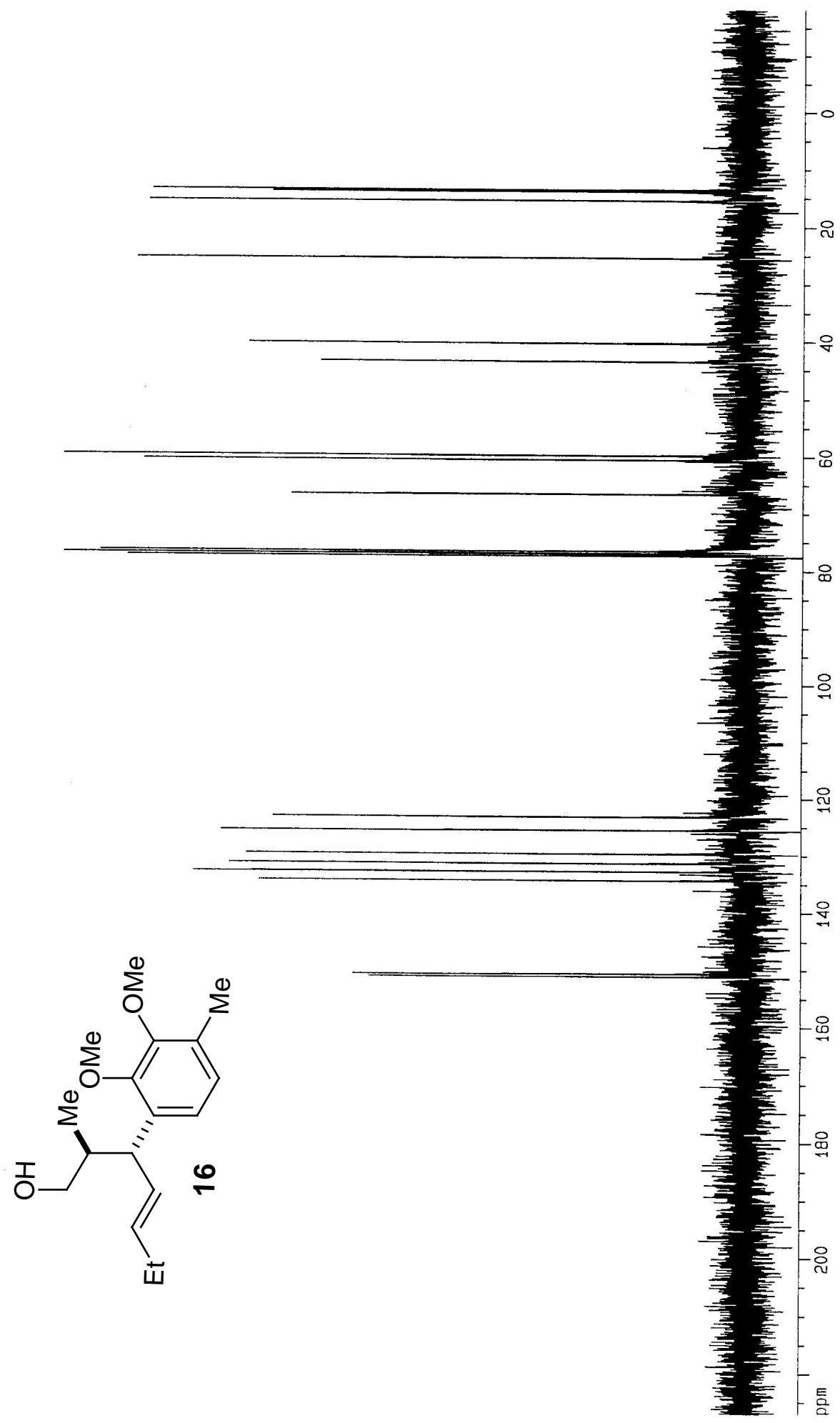

wdd 

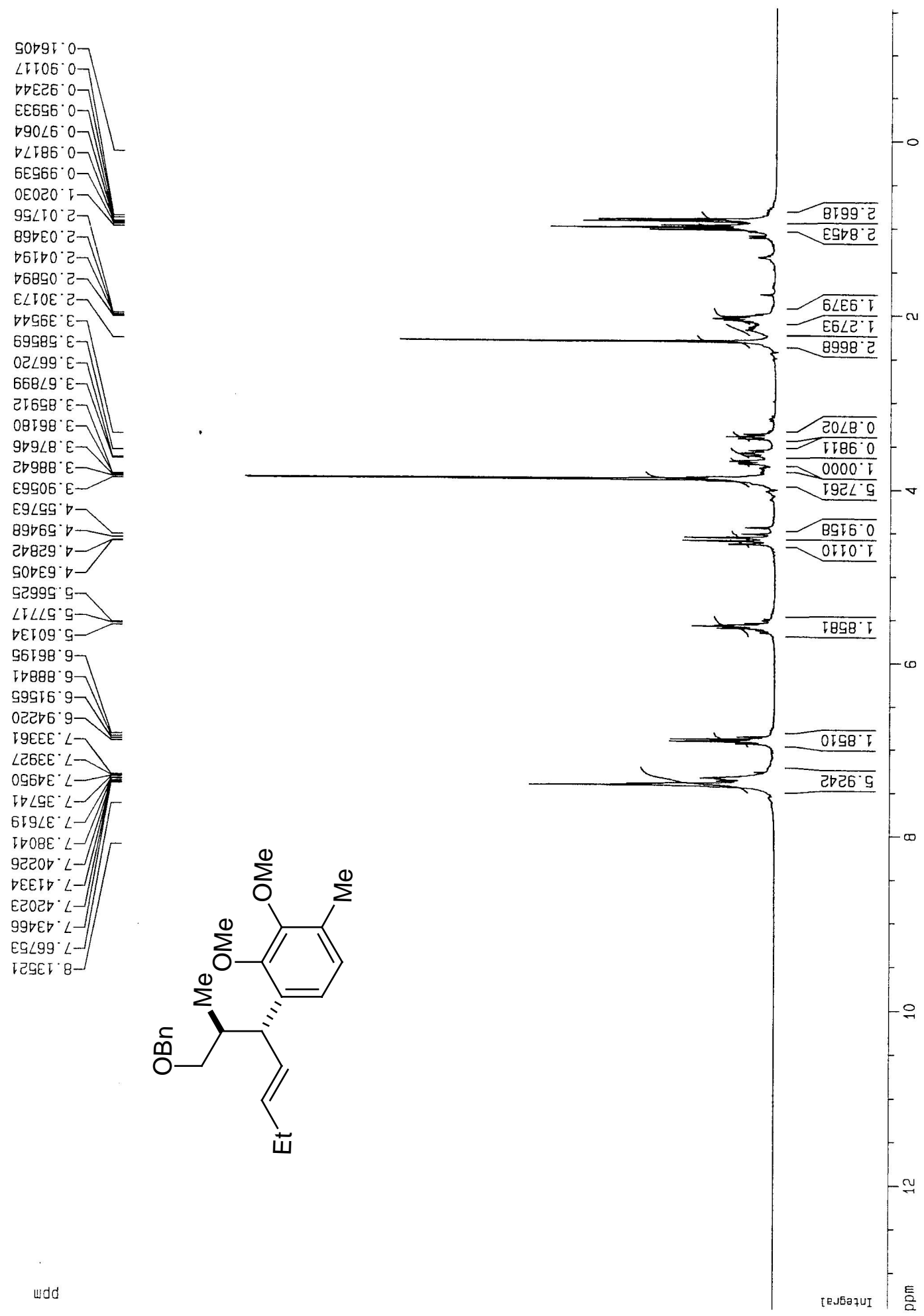
$E I L E E$

टा9.

DOट. 91

פ6巾. 92

$B E B^{\circ} \angle E$

$O D E^{\prime} \mathrm{G} \longrightarrow$

$\angle 8 \angle{ }^{\circ} 69-$

$\angle E E^{\circ} 09$

$\nabla 86^{\circ} \mathrm{CL}$

$081^{\circ} \nabla L \longrightarrow$

$9 \angle 9^{\circ} 9 \angle$

$000^{\circ} \angle L-1$

$2 ट \nabla \cdot \angle L$
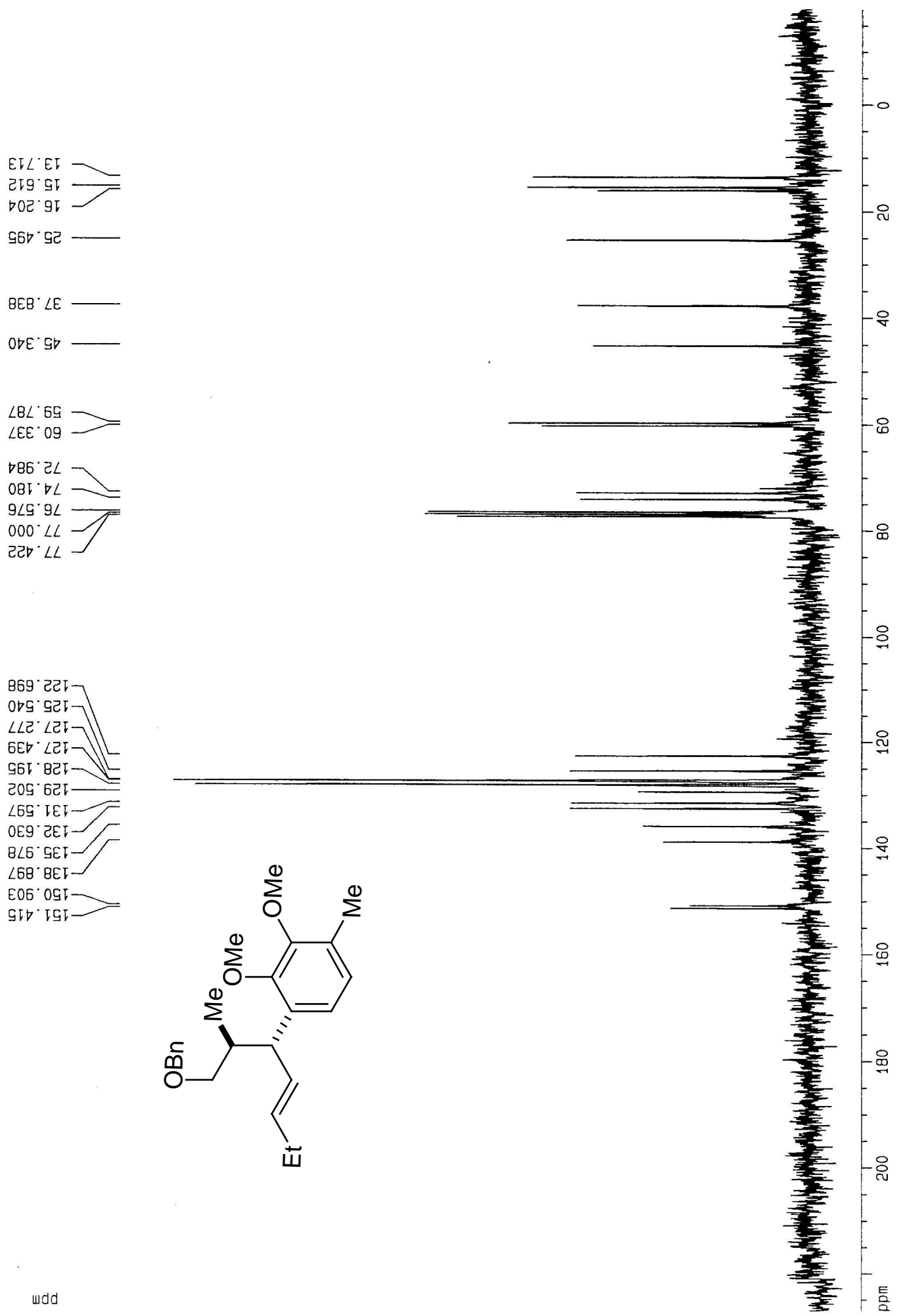


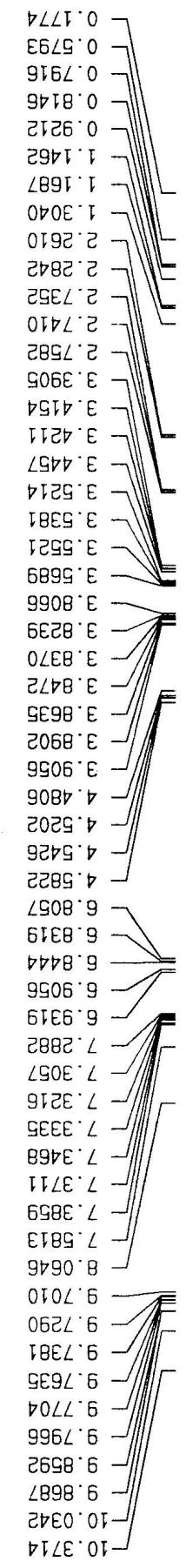

wdd

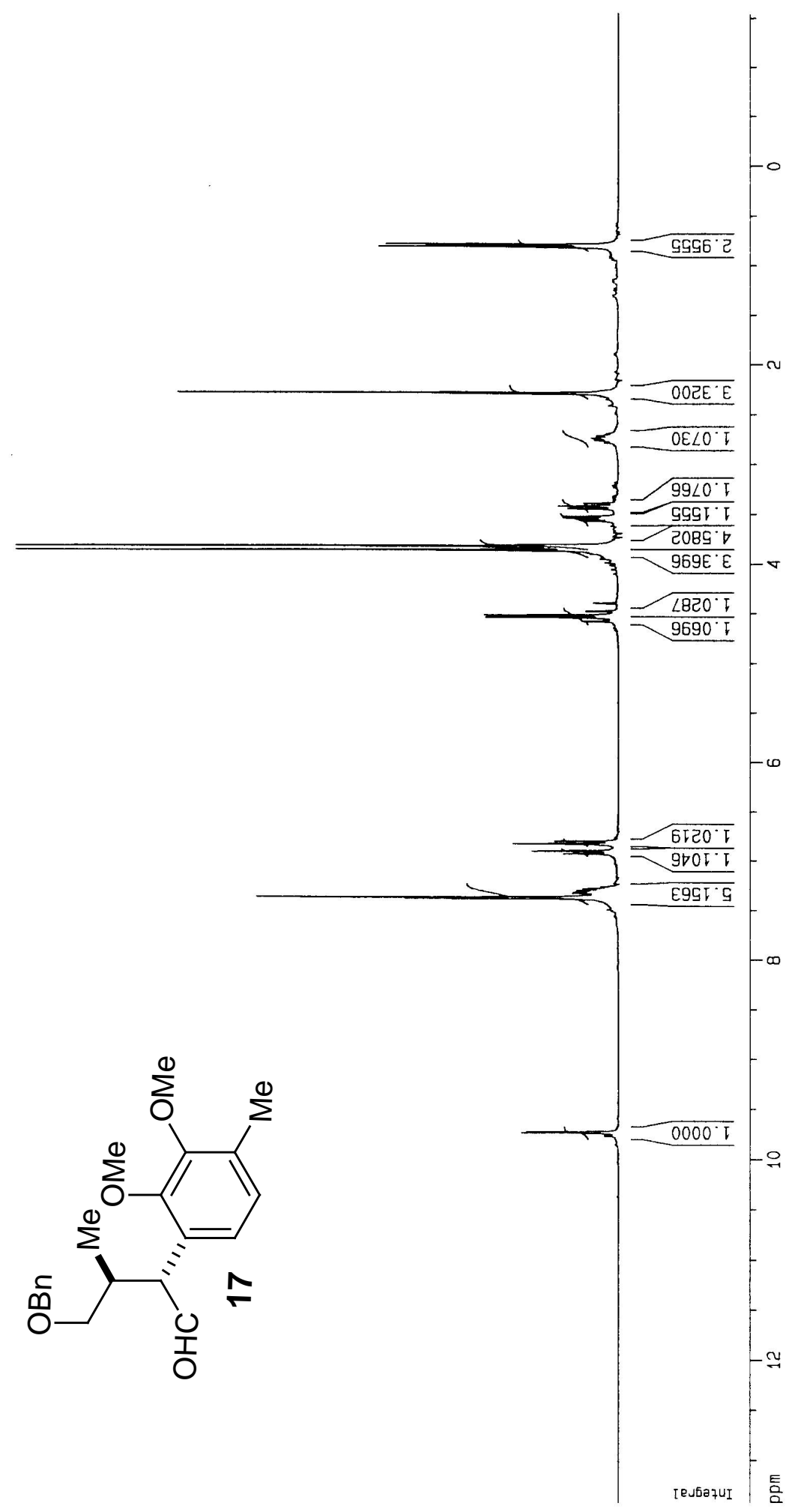


I6G $จ \tau$

II9.

$990^{\circ} \mathrm{DE}$

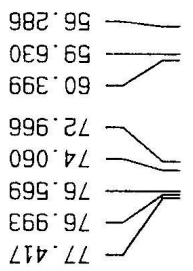

I $\angle S^{\circ} b 2 I-$

OE9 Gटा一

696. 951

$\checkmark \angle C^{\circ} \angle 2 I-$

OEV $\angle 2 T$

695 $\angle 2 T$

EEट Bटा

G9L IEI

BBI $8 E I$

$B \angle b^{\circ}$ 诃 $669^{\circ}$ ISI $>$

GEE' 002 -
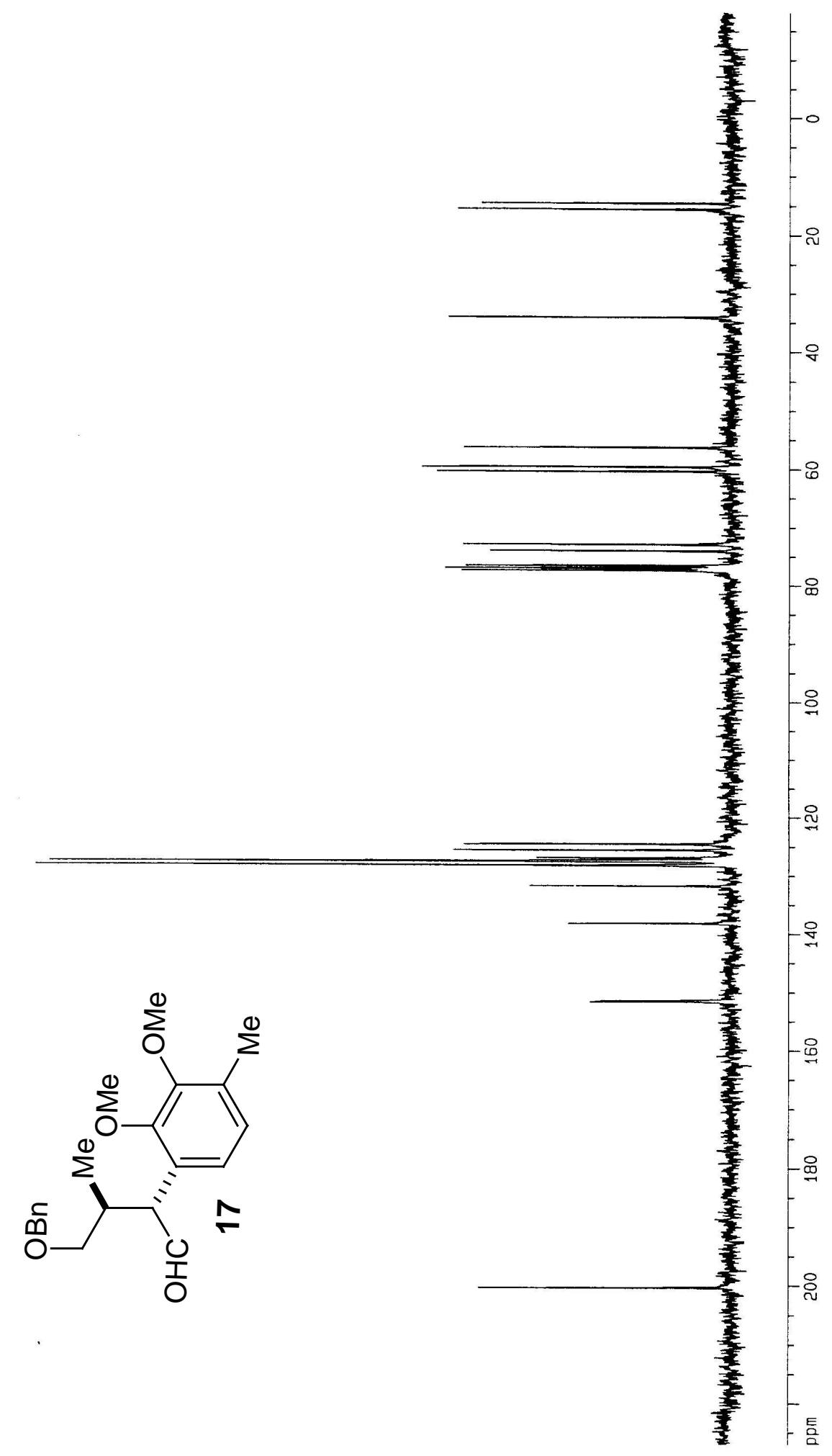

wdd 


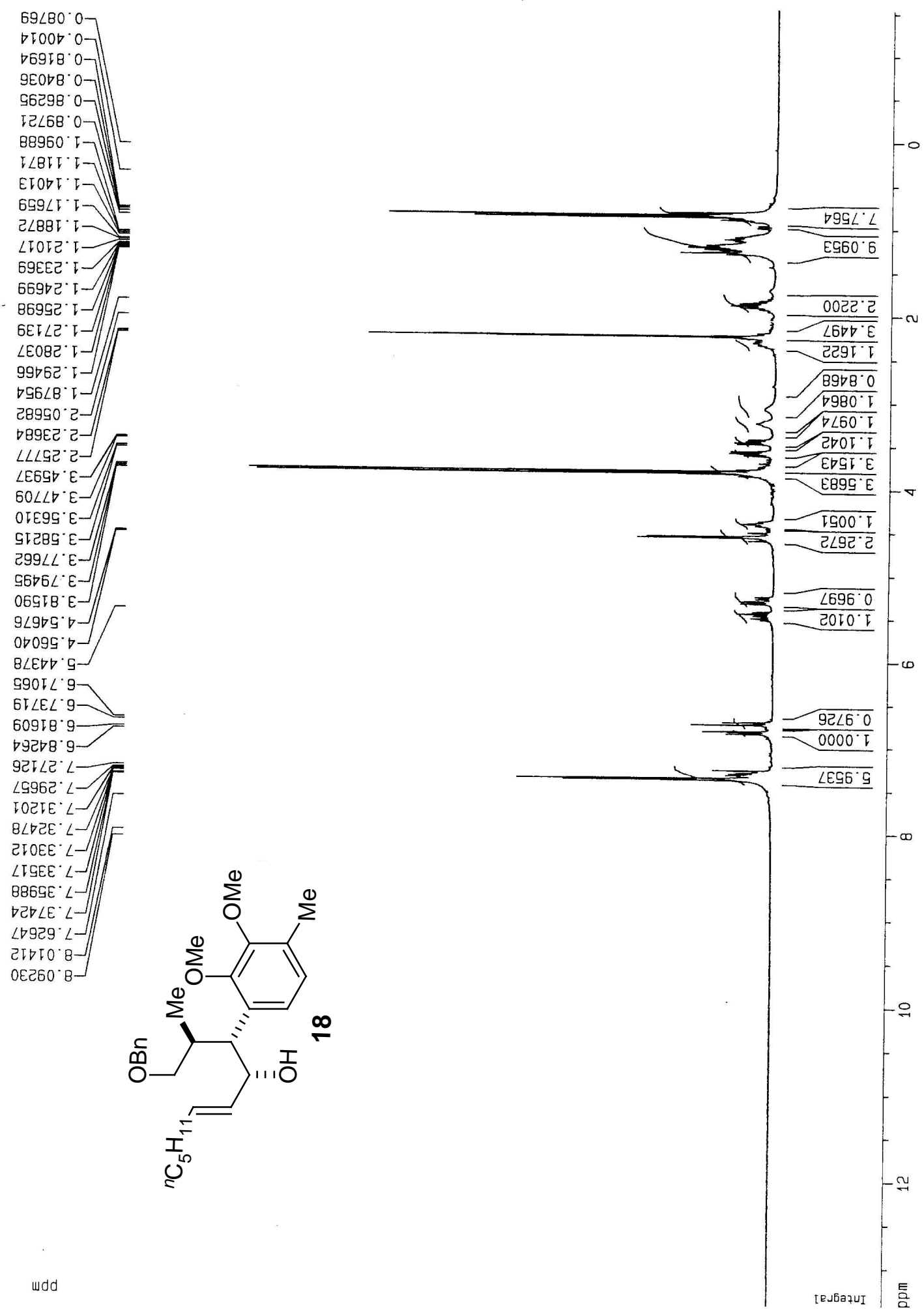



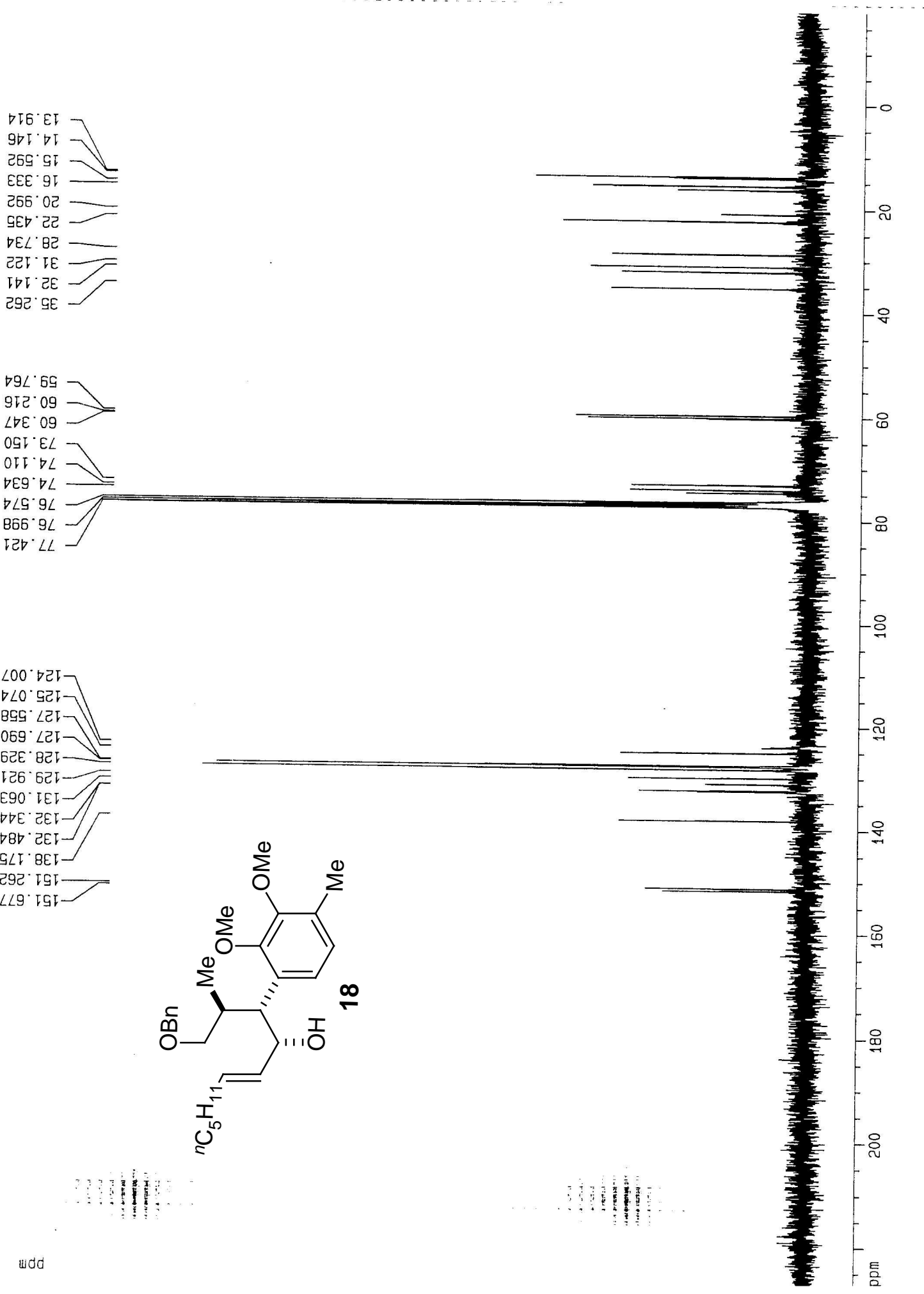

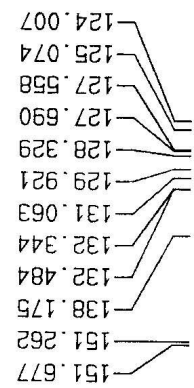

$\nabla 9 \angle \cdot 69-$

9L地 $09 \longrightarrow$

$\angle \forall E^{\circ} 09$

OGI'EL

$O I T B L$

$\forall E 9^{\circ} \circ L$

$\nabla \angle 9^{\circ} 9 \angle$

Iटt $\angle L$

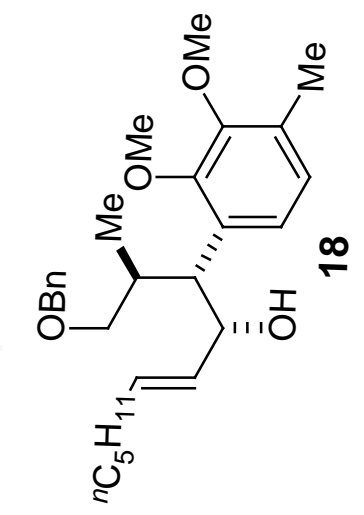

3

31

wod 


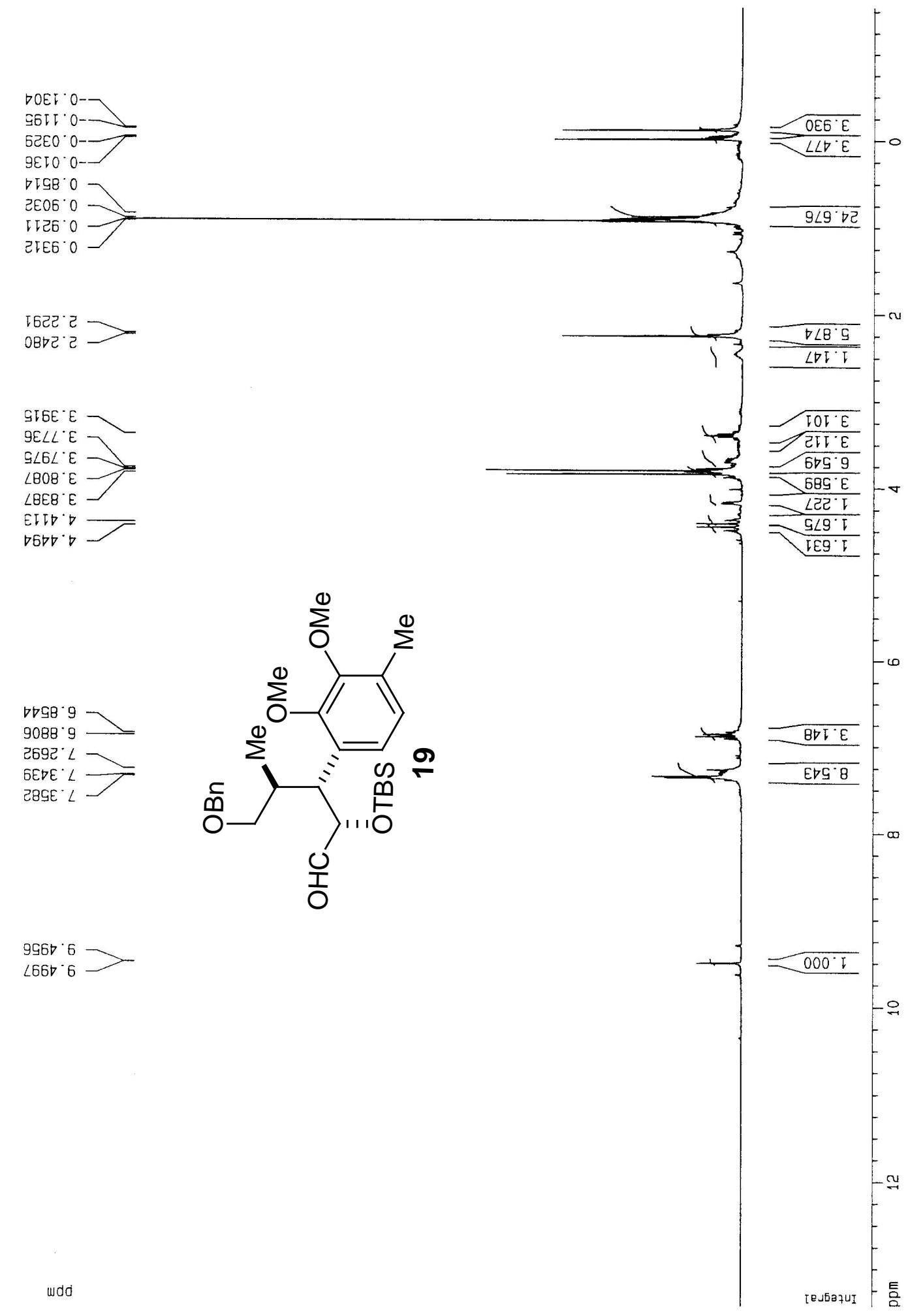




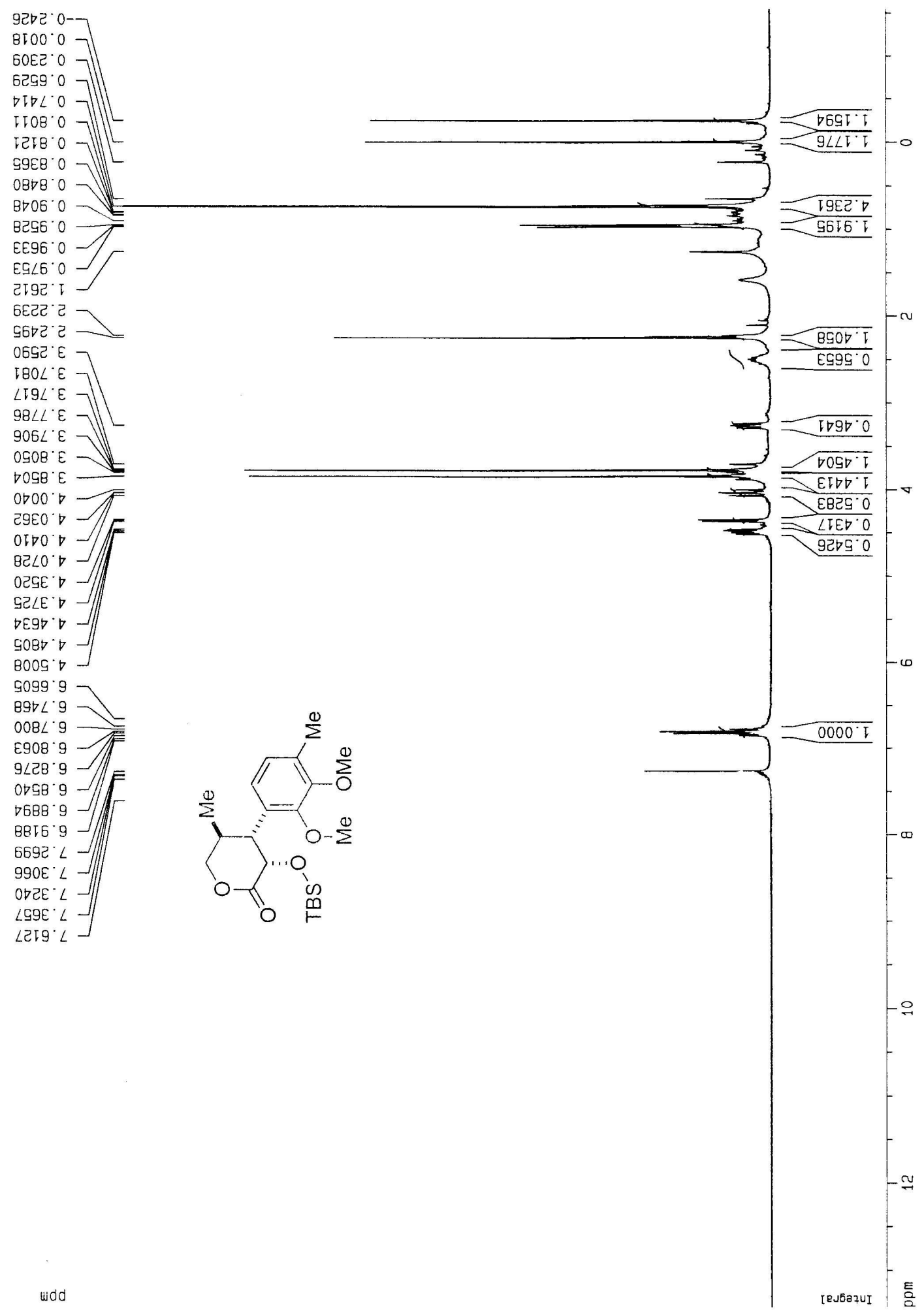



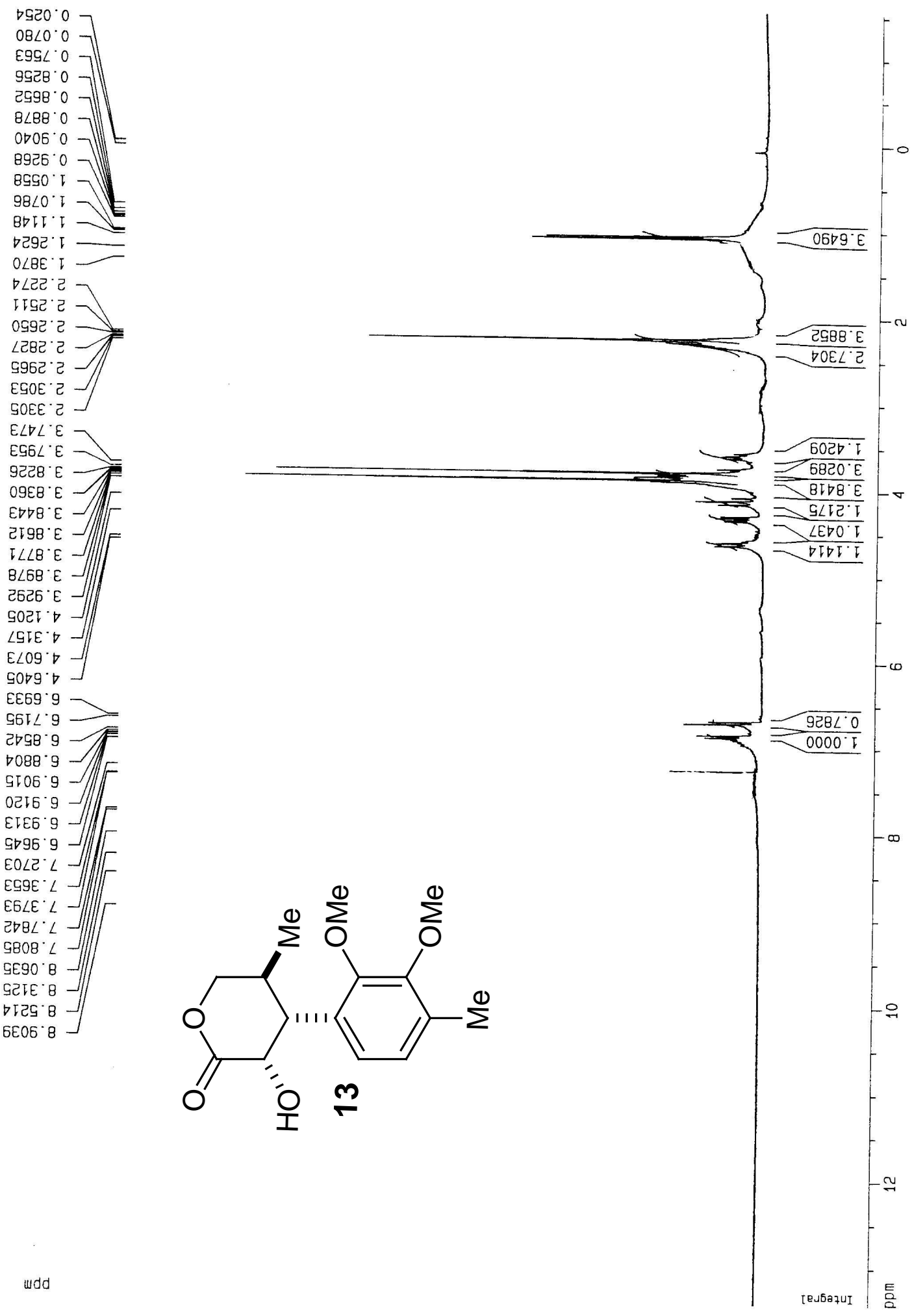

udd 
$B E L \cdot G I$

$000 \cdot 91>$

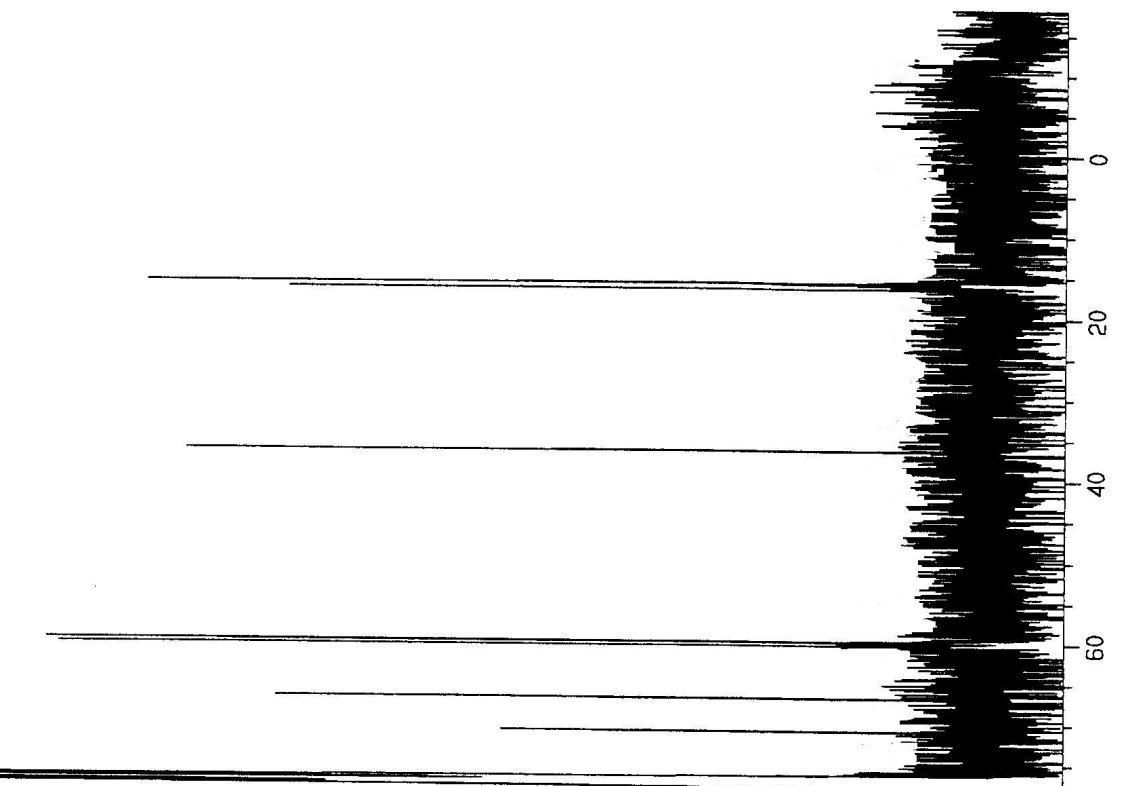

6EE'9E -

$29 L .69$
$802 \cdot 09-$
$5 L \angle \cdot 99-$
$018 \cdot 0 L$
$2 \angle 9 \cdot 9 L$
$566 \cdot 9 L$
$86 L \cdot \angle L$
$6 L V \cdot \angle L$

981

$\angle 90^{\circ}$ Gटा

DDI OEI-

$\checkmark \angle 5^{\circ}$ IEI -

9L6. OGI 896. ISI

$\angle 20^{\circ} G \angle L$<smiles>C#Cc1ccc(C2C(=O)C(=O)OC[C@@H]2C#C)c(O)c1O</smiles>

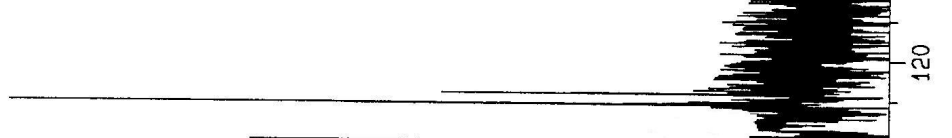

